\title{
Spatial proteogenomics reveals distinct and evolutionarily conserved hepatic macrophage niches
}

\section{Graphical abstract}

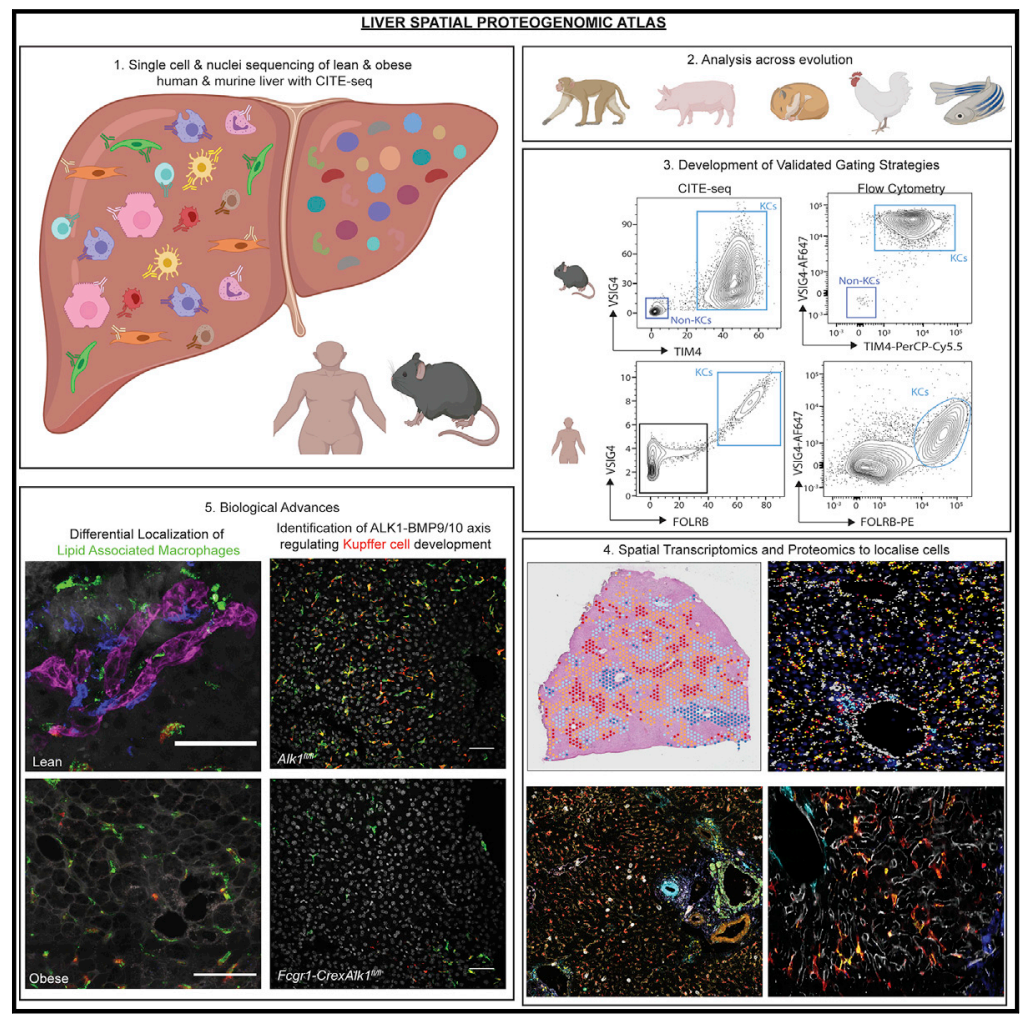

\section{Highlights}

- Spatial proteogenomic single-cell atlas of healthy and obese murine and human liver

- Validated flow cytometry and microscopy panels for all hepatic cells

- LAMs are differentially located in the lean and obese liver

- Evolutionary conserved BMP9/10-ALK1 axis is essential for KC development

\section{Authors}

Martin Guilliams, Johnny Bonnardel, Birthe Haest, ..., Hans Van Vlierberghe, Lindsey Devisscher, Charlotte L. Scott

\section{Correspondence}

martin.guilliams@ugent.be (M.G.), charlotte.scott@ugent.be (C.L.S.)

\section{In brief}

By combining single-cell and -nucleus sequencing with spatial mapping of RNA and proteins, this vast spatial proteogenomic atlas of healthy and obese human and mouse livers presents methods to identify and localize all hepatic cells and provides insights into hepatic myeloid cells, including identification of reliable surface markers for isolation and localization of hepatic macrophages, characterization of lipidassociated macrophages in both healthy and steatotic livers, determination of a key regulatory axis of Kupffer cell development, and identification of a conserved core gene expression signature of Kupffer cells across 7 species, including chickens and zebrafish. 


\title{
Spatial proteogenomics reveals distinct and evolutionarily conserved hepatic macrophage niches
}

\author{
Martin Guilliams, ${ }^{1,2,23,25, *}$ Johnny Bonnardel, ${ }^{1,2,23}$ Birthe Haest, ${ }^{1,2,23}$ Bart Vanderborght, ${ }^{3,4,23}$ Camille Wagner, ${ }^{1,2}$ \\ Anneleen Remmerie, ${ }^{2,5}$ Anna Bujko, ${ }^{2,5}$ Liesbet Martens, ${ }^{1,2,5}$ Tinne Thoné, ${ }^{1,2,5}$ Robin Browaeys, ${ }^{6,7}$ Federico F. De Ponti, ${ }^{2,5}$ \\ Bavo Vanneste, ${ }^{1,2,5}$ Christian Zwicker, ${ }^{2,5}$ Freya R. Svedberg, ${ }^{1,2}$ Tineke Vanhalewyn, ${ }^{1,2,5}$ Amanda Gonçalves, ${ }^{2,8}$ \\ Saskia Lippens, ${ }^{2,8}$ Bert Devriendt, ${ }^{9}$ Eric Cox, ${ }^{9}$ Giuliano Ferrero, ${ }^{10}$ Valerie Wittamer, ${ }^{10,11,12}$ Andy Willaert, ${ }^{13}$
}

(Author list continued on next page)

\begin{abstract}
${ }^{1}$ Laboratory of Myeloid Cell Biology in Tissue Homeostasis and Regeneration, VIB-UGent Center for Inflammation Research, Technologiepark-Zwijnaarde 71, Ghent 9052, Belgium

2Department of Biomedical Molecular Biology, Faculty of Science, Ghent University, Belgium

${ }^{3}$ Hepatology Research Unit, Department Internal Medicine and Pediatrics, Liver Research Center, Ghent University, Belgium

${ }^{4}$ Gut-Liver Immunopharmacology Unit, Department of Basic and Applied Medical Sciences, Liver Research Center, Ghent University, Belgium

${ }^{5}$ Laboratory of Myeloid Cell Biology in Tissue Damage and Inflammation, VIB-UGent Center for Inflammation Research, Technologiepark-Zwijnaarde 71, Ghent 9052, Belgium

${ }^{6}$ Data Mining and Modelling for Biomedicine, VIB-UGent Center for Inflammation Research, Technologiepark-Zwijnaarde 71, Ghent 9052, Belgium

${ }^{7}$ Department of Applied Mathematics, Computer Science and Statistics, Faculty of Science, Ghent University, Ghent, Belgium

8VIB Biolmaging Core, VIB-UGent Center for Inflammation Research, Technologiepark-Zwijnaarde 71, Ghent 9052, Belgium

'Laboratory of Immunology, Department of Translational Physiology, Infectiology and Public Health, Faculty of Veterinary Medicine, Ghent University, Belgium

${ }^{10}$ Institut de Recherche Interdisciplinaire en Biologie Humaine et Moléculaire (IRIBHM), Université Libre de Bruxelles (ULB), Brussels, Belgium ${ }^{11}$ ULB Institute of Neuroscience (UNI), Université Libre de Bruxelles (ULB), Brussels, Belgium

12WELBIO, Université Libre de Bruxelles (ULB), Brussels, Belgium

${ }^{13}$ Center for Medical Genetics Ghent, Department of Biomolecular Medicine, Ghent University, Ghent, Belgium

${ }^{14} \mathrm{KU}$ Leuven Department of Microbiology, Immunology and Transplantation, Rega Institute, Laboratory of Virology and Chemotherapy, Molecular Vaccinology and Vaccine Discovery, Leuven, Belgium

${ }^{15}$ Laboratory of Parasitology, Department of Translational Physiology, Infectiology and Public Health, Faculty of Veterinary Medicine, Ghent University, Ghent, Belgium
\end{abstract}

(Affiliations continued on next page)

\section{SUMMARY}

The liver is the largest solid organ in the body, yet it remains incompletely characterized. Here we present a spatial proteogenomic atlas of the healthy and obese human and murine liver combining single-cell CITEseq, single-nuclei sequencing, spatial transcriptomics, and spatial proteomics. By integrating these multiomic datasets, we provide validated strategies to reliably discriminate and localize all hepatic cells, including a population of lipid-associated macrophages (LAMs) at the bile ducts. We then align this atlas across seven species, revealing the conserved program of bona fide Kupffer cells and LAMs. We also uncover the respective spatially resolved cellular niches of these macrophages and the microenvironmental circuits driving their unique transcriptomic identities. We demonstrate that LAMs are induced by local lipid exposure, leading to their induction in steatotic regions of the murine and human liver, while Kupffer cell development crucially depends on their cross-talk with hepatic stellate cells via the evolutionarily conserved ALK1-BMP9/10 axis.

\section{INTRODUCTION}

The immense advances in single-cell transcriptomics have enabled a better understanding of the cellular composition of different organs across species. However, we still lack information regarding how these cells are organized in their distinct microenvironmental niches. Moreover, the specific cell-cell interactions determining the identity of individual cells within 
Suzanne J.F. Kaptein, ${ }^{14}$ Johan Neyts, ${ }^{14}$ Kai Dallmeier, ${ }^{14}$ Peter Geldhof, ${ }^{15}$ Stijn Casaert, ${ }^{15}$ Bart Deplancke, ${ }^{16,17}$ Peter ten Dijke, ${ }^{18}$ Anne Hoorens, ${ }^{19}$ Aude Vanlander, ${ }^{20}$ Frederik Berrevoet, ${ }^{20}$ Yves Van Nieuwenhove, ${ }^{21}$ Yvan Saeys, ${ }^{6,7}$ Wouter Saelens, ${ }^{16,17,24}$ Hans Van Vlierberghe, ${ }^{3,22,24}$ Lindsey Devisscher, ${ }^{4,24}$ and Charlotte L. Scott ${ }^{2,5,24, *}$

${ }^{16}$ Laboratory of Systems Biology and Genetics, Institute of Bioengineering, School of Life Sciences, École Polytechnique Fédérale de Lausanne (EPFL), Lausanne, Switzerland

${ }^{17}$ Swiss Institute of Bioinformatics (SIB), Lausanne, Switzerland

${ }^{18}$ Oncode Institute, Department of Cell and Chemical Biology, Leiden Medical Center, Leiden, Netherlands

${ }^{19}$ Department of Pathology, Ghent University Hospital, Ghent 9000, Belgium

${ }^{20}$ Department of General and Hepatopancreatobiliary Surgery and Liver Transplantation, Ghent University Hospital, Ghent 9000 , Belgium

${ }^{21}$ Department of Human Structure and Repair, Ghent University Hospital, Ghent 9000, Belgium

${ }^{22}$ Department of Gastroenterology and Hepatology, Ghent University Hospital, Ghent 9000, Belgium

${ }^{23}$ These authors contributed equally

24These authors contributed equally

${ }^{25}$ Lead contact

*Correspondence: martin.guilliams@ugent.be (M.G.), charlotte.scott@ugent.be (C.L.S.)

https://doi.org/10.1016/j.cell.2021.12.018

tissues remain to be defined (Guilliams and Scott, 2017; Lindeboom et al., 2021). While the spatial organization of hepatocytes within the liver is understood (Halpern et al., 2017), that of nonparenchymal liver cells remains unclear. This is the case for the mouse liver, but even more so for the human liver, where the identity and the precise localization of most hepatic cells is unknown. Moreover, the link between the transcriptome and the proteome has not been studied, resulting in a lack of reliable surface markers to identify these cells by flow cytometry and confocal microscopy. Here, we used proteogenomic techniques including cellular indexing of transcriptomes and epitomes by sequencing (CITE-seq) and spatial approaches to identify all cells and their specific locations within the healthy and obese livers of mice and humans. By doing so, we have developed strategies for the identification and further study of hepatic cells. Demonstrating the usefulness of this approach, we identify the evolutionary conserved and spatially restricted signals driving the distinct hepatic macrophage phenotypes.

\section{RESULTS}

A practical proteogenomic atlas of the murine liver To generate a proteogenomic atlas of the liver, we first examined the optimal method for retrieving all hepatic cells. Using the murine liver, we compared single-cell RNA sequencing (scRNAseq) using cells isolated via ex vivo or in vivo enzymatic digestion with single-nuclei RNA sequencing (snRNA-seq) (Figures S1A$\mathrm{S} 1 \mathrm{C})$. We did not observe any differences in the number of genes/cell between the two digestion methods (Figures S1D and S1E), but snRNA-seq typically yielded a lower number of genes/cell (Figures S1D and S1F). This did not prevent distinct cell types from being identified in the snRNA-seq dataset as both scRNA-seq and snRNA-seq identified highly expressed genes in each population. However, expression was often higher in the scRNA-seq (Figure S1F). Additionally, we observed a signature of digestion-associated genes and snRNA-seq-associated genes across cell types (Figure S1F). While in terms of genes/cell snRNA-seq is inferior to scRNA-seq, this method best recapitulated the cell frequencies observed in vivo (Figures S1D-S1N). As each method has advantages and disadvantages, the optimal method to use depends on the biological question being addressed. Here, as we sought to generate a proteogenomic atlas of all hepatic cells, we thus opted to use a combination of all protocols. To investigate mRNA and protein expression at single-cell resolution, we used CITE-seq (Stoeckius et al., 2017), staining a selection of the scRNA-seq samples with 107-161 oligo-conjugated antibodies (Figure 1A). Data were pooled together for a single analysis where, with TotalVI (Gayoso et al., 2021), both the protein and mRNA profiles were considered for clustering (Figure 1B). Analysis of the differentially expressed genes and proteins (DEGs/DEPs; Figures S2A and S2B; Table S1) identified 17 cell types (Figure 1B), which were differentially represented with each isolation method (Figure S2C). Addition of antibodies in the CITE-seq analysis enabled surface markers for all cells to be identified, including VSIG4 and FOLR2 for Kupffer cells (KCs) (Figure S2B), without affecting the quality of the transcriptomic data (Figure 2D). This analysis identified one subset of KCs. However, 2 subpopulations termed $\mathrm{KC} 1$ and $\mathrm{KC} 2$ have recently been described (Blériot et al., 2021; Simone et al., 2021). As our CITE-seq analysis identified that the markers used to identify KC2s, namely CD206 and ESAM, are largely expressed by liver sinusoidal endothelial cells (LSECs) (Figure S2E), we next sought to determine if we had previously removed any potential KC2s in our initial QC steps as LSEC-KC doublets. To examine this, we generated a UMAP of samples containing CD206 and ESAM in the CITE-seq panel and performed a minimal QC filtering on number of genes and percentage of mitochondrial genes. In this UMAP, we found multiple subpopulations including 3 populations of KCs and 2 populations of B cells (Figure S2F). To determine if any of these populations could be $\mathrm{KC} 2 \mathrm{~s}$, we harnessed the power of CITE-seq to recreate the gating strategy recently proposed to identify KC2s (Blériot et al., 2021). Converting the CITE-seq data into an FCS file and analyzing this in FlowJo identified 2 of the $\mathrm{KC}$ populations to be KC2s expressing CD206 and ESAM, and these cells were present in a similar ratio among $\mathrm{KCs}$ as reported by Blériot et al. (Figure S2G). However, employing the same gating strategy with the B cells also enabled a B cell2 population to be identified (Figure S2G). The KC2 and B cell2 populations also expressed other protein markers associated with LSECs including CD26, CD31, and CD38, suggesting that these may be doublets (Figure $\mathrm{S} 2 \mathrm{H})$. Consistent with this, we did not uncover any DEGs 
A

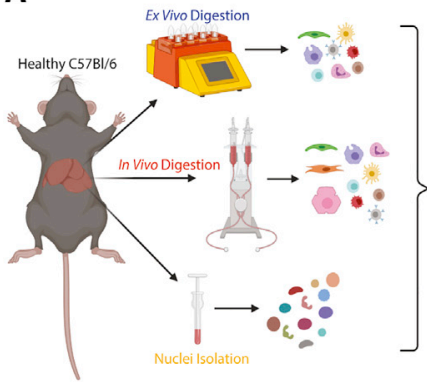

D
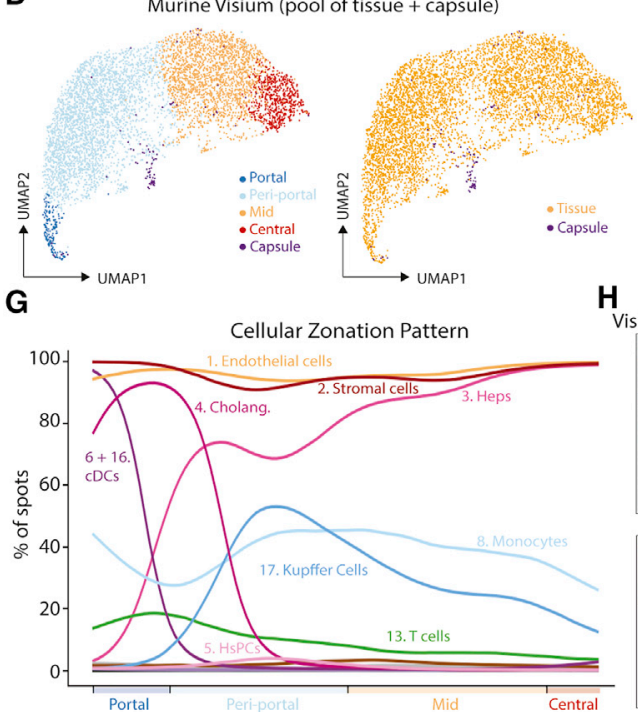

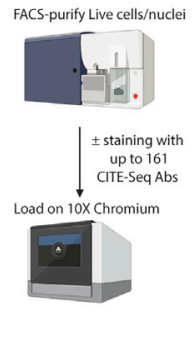

B

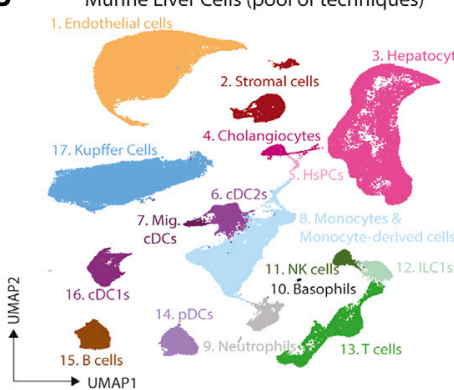

E

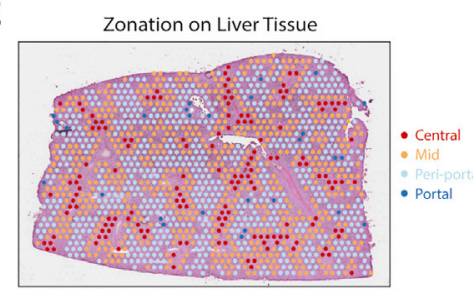

C

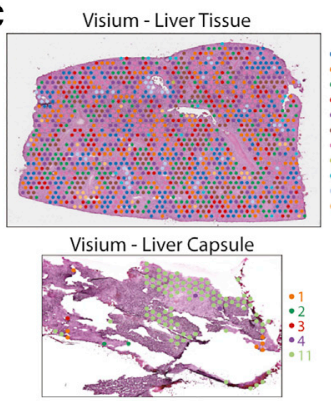

$\mathbf{F}$

F Location of KC signature

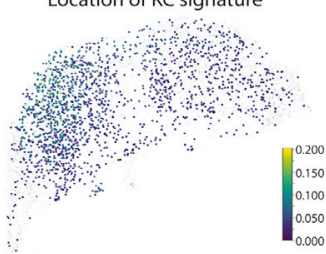
Visium Highly-Multiplexed Protein
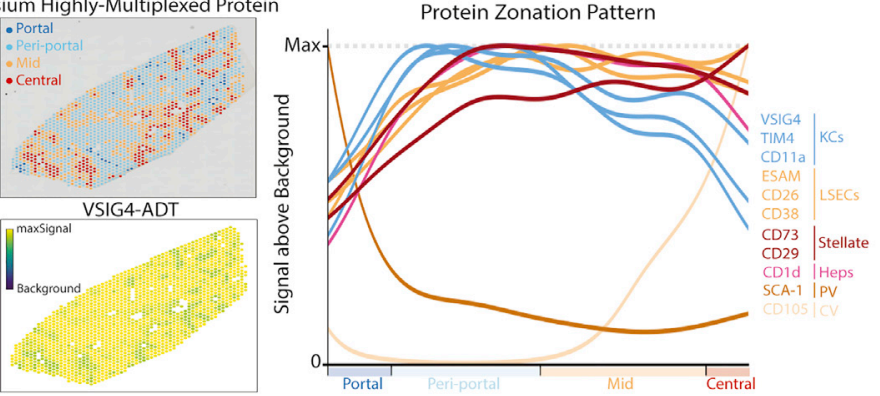

I 60-Plex Protein (MICSTechnology) J

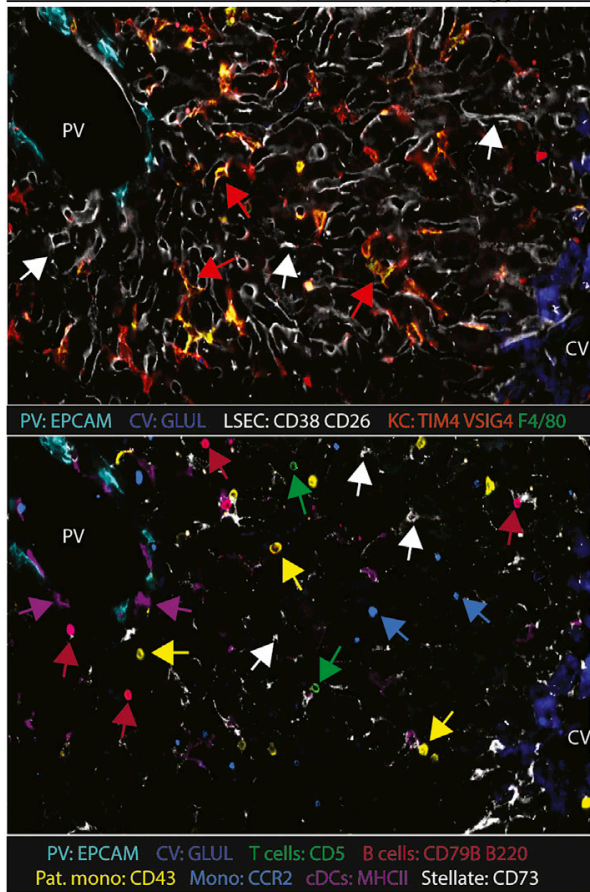

100-plex mRNA (Molecular Cartography ${ }^{\mathrm{TM}}$ )
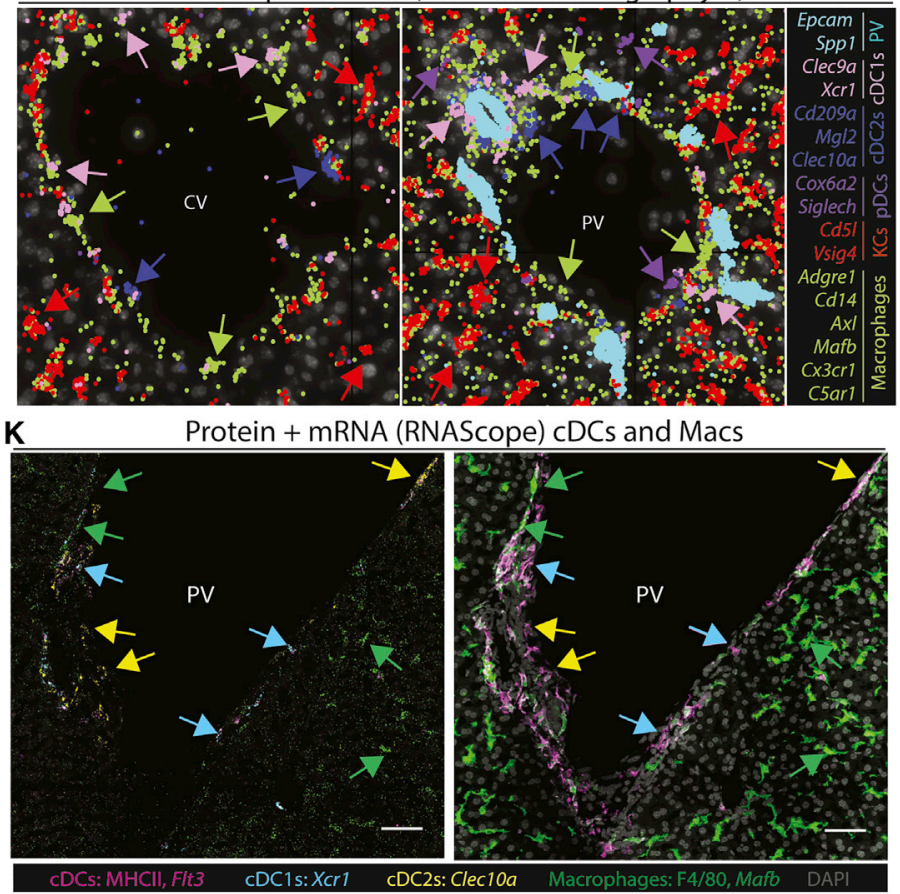

(legend on next page) 
specifically expressed in the KC2s or B cell2s, rather these cells had an intermediate profile between either KC1s or B cell1s and LSECs (Figure S2I), as would be expected for doublets. Finally, we perfused the livers with antigen fix to inflate the LSECs to be able to distinguish more readily between KCs and LSECs and performed confocal microscopy. 3D reconstruction of these images indicates that CD206 expression is observed primarily in the LSECs with which the KCs are intertwined. These microscopy images also show that KCs express some CD206, however, consistent with our CITE-seq analysis, this was observed across the $\mathrm{KC}$ population rather than in a $\mathrm{KC}$ subset further suggesting the existence of only one-KC population (Figure S2J). With this in mind, we decided to continue to apply our initial strict QC controls eliminating these potential doublets from further analyses.

\section{Distinct spatial orientation of hepatic myeloid cell subsets}

To locate the cells identified we performed spatial transcriptomics analysis using Visium. For this, we cut the liver in two distinct orientations to profile both the liver tissue and the capsule (Figures $1 \mathrm{C}$ and $\mathrm{S} 2 \mathrm{~K})$. We ordered each Visium spot along a spatial trajectory, and annotated portal, periportal, mid, and central zones based on known hepatocyte zonation markers (Halpern et al., 2017; Figures 1D, 1E, and S2L) and confirmed this annotation using confocal microscopy (Figure S2M). By using the reference sc/snRNA-seq data, we then deconvolved each spot into its constituent cell types and investigated how cell abundance changed with zonation (Figures 1F, 1G, and S2N). Validating this approach, cholangiocytes mapped specifically to the portal zones (Aizarani et al., 2019), while KCs were preferentially located in periportal and mid zones (Bonnardel et al., 2019; Gola et al., 2021). While KC location is zonated, we did not identify a strong zonation pattern in the gene expression profiles of KCs (data not shown). We further identified B cells, T cells, endothelial cells (ECs), and stromal cells (SCs) across all zones, while conventional dendritic cells (cDCs) were found at the portal vein $(\mathrm{PV})$, with a minor presence at the central vein (CV) (Figures 1F, $1 \mathrm{G}$, and $\mathrm{S} 2 \mathrm{~N}$ ).

To validate these locations at single-cell resolution, we next sought to identify the best cell-specific surface markers that would also work by confocal microscopy. As the fixation step utilized for confocal microscopy often affects the integrity of protein epitopes, it is not possible to predict which antibodies will work spatially on fixed tissue slices. Therefore, to simultaneously screen multiple antibodies to identify those working by microscopy, we performed a second Visium analysis which we complemented with 100 oligo-conjugated antibodies, chosen based on the CITE-seq results (Figure $1 \mathrm{H}$ ). The antibodies identified to work spatially were then validated at single-cell resolution, using MACSima $^{\mathrm{TM}}$ Imaging Cyclic Staining (MICS) technology and a 60-plex antibody panel (Figure 1I). Unfortunately, we could not identify useful surface markers for all populations. For example, we did not identify enough discriminatory surface markers that worked by confocal microscopy to distinguish the CDC subsets. Notably, while CD103 has previously been proposed to distinguish hepatic cDC1s from cDC2s (Eckert et al., 2015), our data demonstrate that this is only expressed by a fraction of these cells (Figure S2O). To confirm the locations of the cDC subsets, we thus turned to Molecular Cartography ${ }^{\mathrm{TM}}$ (Resolve BioSciences) that allows for 100-plex spatial mRNA analysis. Genes were selected based on the DEGs from the sc/snRNA-seq data that were also spatially resolved according to Visium. We also identified the portal-central trajectory in this dataset using cholangiocytes genes (Epcam and Spp1) and known zonated hepatocyte genes (Glul, Cyp2e1, Hal, and Sds; Figure S2P). Using expression of $\mathrm{Xcr} 1$, Clec9a (cDC1s) and Cd209a, Mgl2, and Clec10a (cDC2s), we confirmed that both cDC1s and cDC2s were localized primarily at the PV (Figure 1J). As CDC2s shared a number of genes with monocyte-derived cells (Figure S2A), we also examined the expression of general monocyte/mac (Cd14, Adgre1, Axl, Mafb, Cx3cr1, and C5ar1) and KC-specific genes (Cd5/ and Vsig4) to further validate their identification as bona fide cDC2s (Figure 1J). The punctate nature of mRNA expression in these analyses combined with the dendritic shape of myeloid cells renders it difficult to convincingly determine cell boundaries and to conclude these cDC2s and macs were distinct cells. To validate this, we therefore developed a protoco that combines mRNA detection (RNAScope) with surface protein detection. Examining expression of cDC- or mac-specific mRNAs combined with protein surface markers confirmed the presence of PV cDC1, cDC2s, and non-KC macs (Figure $1 \mathrm{~K}$ ).

Figure 1. A proteogenomic atlas of the healthy murine liver

(A) Hepatic cells were isolated from healthy C57B/I6 mice by ex vivo ( 5 mice, 15 samples) or in vivo ( 5 mice, 19 samples) enzymatic digestion. Alternatively, nuclei were isolated by tissue homogenization (4 mice, 12 samples). Live cells/intact nuclei were FACS-purified. For cells, total live, live CD45 ${ }^{+}$, live CD45 ${ }^{-}$, live hepatocytes, or myeloid cells (live CD45 $\left.{ }^{+}, \mathrm{CD}^{-}, \mathrm{CD} 19^{-}, \mathrm{B} 220^{-}, \mathrm{NK} 1.1^{-}\right)$were sorted. 18 samples $(7$ ex vivo, 11 in vivo) were also stained with a panel of $107-161$ barcode-labeled antibodies for CITE-seq analysis. All datasets were pooled together and after QC 185,894 cells/nuclei were clustered using TotalVI.

(B) UMAP of sc/snRNA-seq data.

(C) Tissue and capsule images from Visium analysis with clusters overlaid.

(D) UMAP of zonation of Visium spots (left) and origin of the cells (right).

(E) Zonation pattern mapped onto tissue slice.

( $F$ and $G$ ) Indicated cell signatures from sc/snRNA-seq mapped onto the Visium zonation data.

(H) mRNA zonation pattern in Visium highly multiplexed protein analysis and VSIG4-ADT expression pattern (left) and zonated expression patterns of indicated antibodies (right).

(I) MICS analysis of indicated proteins.

(J) Molecular Cartography of indicated genes and cell types.

(K) mRNA (Xcr1, Flt3l, Mafb, and Clec10a) and protein (MHCll and F4/80) expression in the same tissue slice. Scale bars, $50 \mu \mathrm{m}$. PV, portal vein; CV, central vein. Arrows indicate specific cell types, colors correspond to cell type/markers. Images are representative of 2-4 mice. See also Figure S2 and Tables S1, S2, S3, and S5. 
A

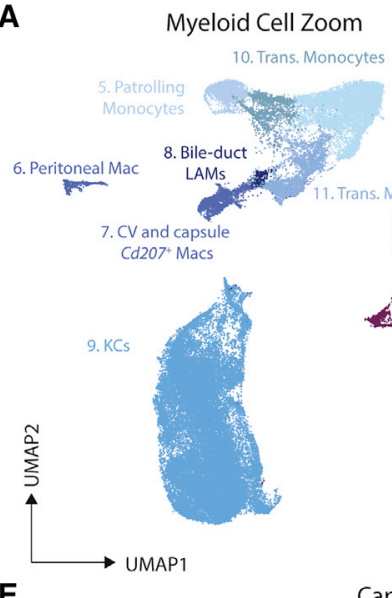

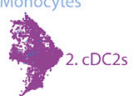

3. Migratory CDCs

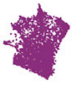

1. CDC1s
B

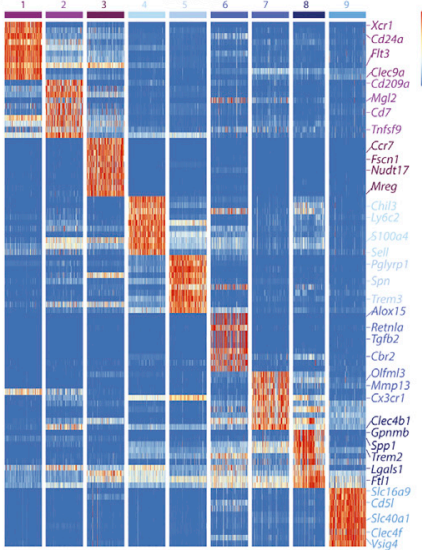

C

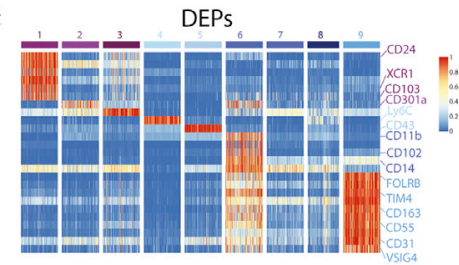

D

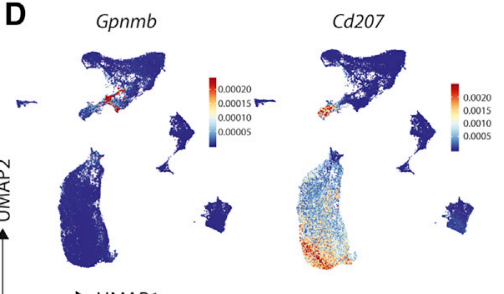

Capsule Macrophages - Protein

F Capsule Macrophages - mRNA

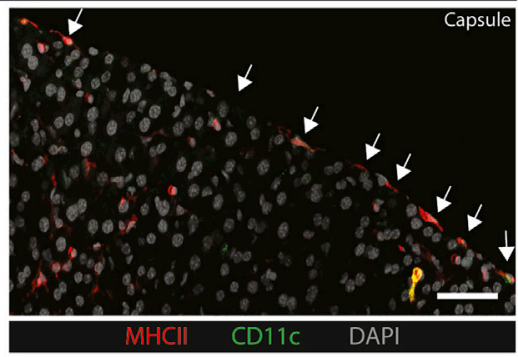

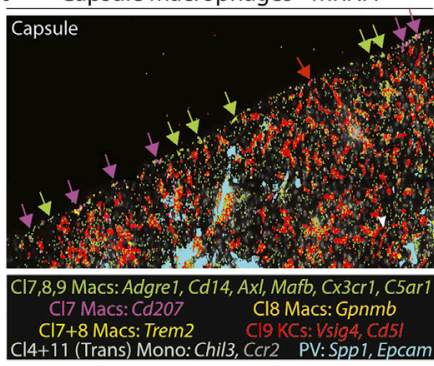

G

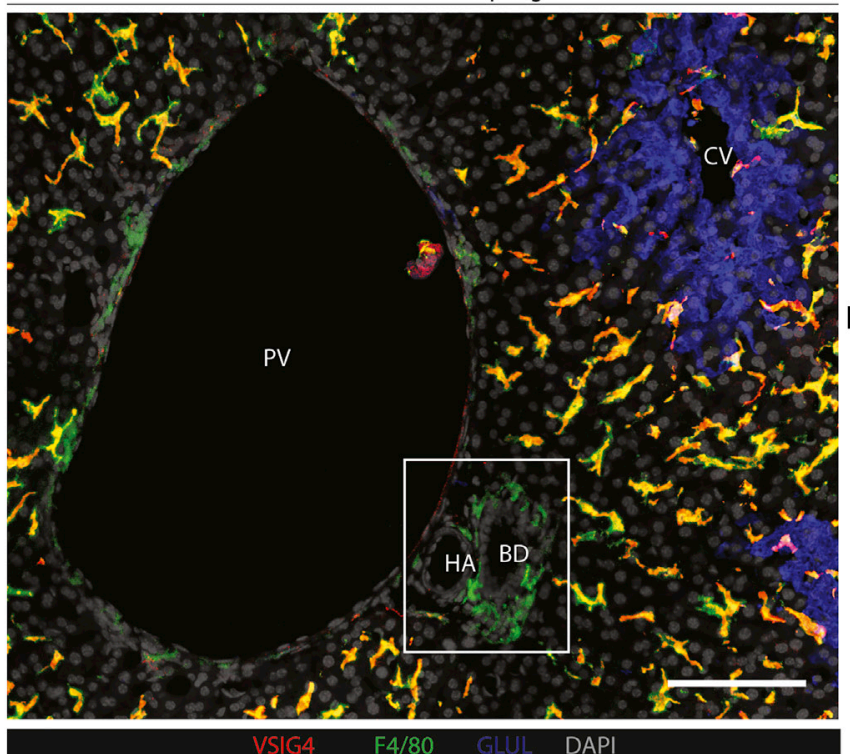

$$
\text { I }
$$

KC GO: Biological Process BD-LAM GO: Biological Process Regulation of humoral immune response Inflammatory response Regulation of complement activation Regulation of immune effector process Cell junction disassembly Synapse prenning Comple tactivation Humo 3 iinmune response inflammatory response Mrelin assembly

Camplement receptor signalling

BD-LAM GO: Biological Process
Inflammatory response
Positive regulation of cell migration
Positive regulation of cell motility
Second-messenger-mediated signalling
Neutrophil degranulation
Neutrophil activation
Neutrophil-mediated immunity
Cellchemotaxis
Positive regulation of [cytosolic calcium ion]
Regulation of [cytosolic calcium ion]

Inset: Bile Duct Macrophages - Protein

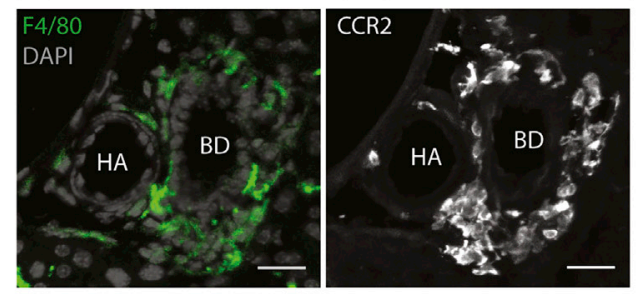

H Portal Vein \& Bile Duct Macrophages - mRNA

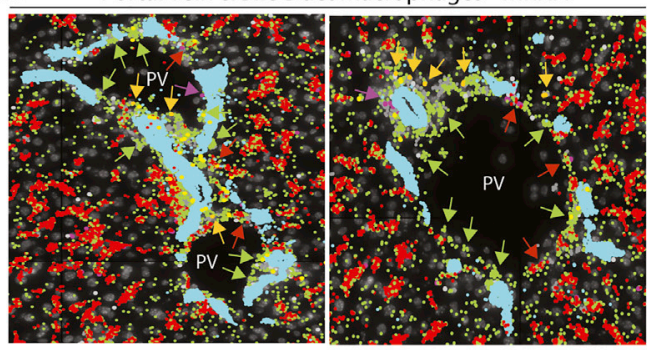

C17,8,9 Macs: Adgre1, Cd14, Axl, Mafb, CX3cr1, C5ar1 C19 KCs: Vsig4, Cd51
C17 Macs: Cd207 Cl8 Macs: Gpnmb Cl7+8 Macs: Trem2 PV:Spp1, Epcam $\mathrm{Cl} 4+11$ (Trans) Mono: $\mathrm{Chil} 3, \mathrm{C} \mathrm{Cr}_{2}$

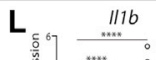

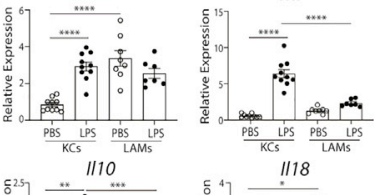

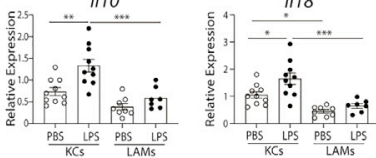


Taken together, by combining multiple spatial transcriptomic and proteomic approaches, we located all the cells within the murine liver and identified additional heterogeneity within the myeloid cells, not revealed when examining the sc/sn-RNAseq dataset in isolation. This highlights the power of combining single-cell and spatial proteogenomic techniques to investigate cellular heterogeneity.

\section{Refined analysis of myeloid cells identifies three} subsets of hepatic macrophages

To better understand the non-KC macs, we zoomed in on myeloid cells (cDCs, KCs, monocytes, and monocyte-derived cells) in our sc/snRNA-seq analysis defining 11 populations (Figures $2 \mathrm{~A}-2 \mathrm{C}$; Table S2). This included KCs, 3 populations of non$\mathrm{KC}$ macs and cells that had a profile intermediate between monocytes and patrolling monocytes or macs, termed transitioning monocytes. Closer inspection of the non-KC macs identified cluster6 as peritoneal macs (Figure 2B). The DEGs between the remaining populations suggested that cluster7 likely resembles capsule macs (Sierro et al., 2017), expressing Cd207 and Cx3cr1 while cluster8 resembles Gpnmb ${ }^{+}$Spp 1+ lipid-associated macrophages (LAMs) we recently described in the fatty liver (Remmerie et al., 2020; Figures 2A-2D). Conversion of the CITEseq data into an FCS file allowed an in silico gating strategy to be defined (Figure S3A). Validating this, we utilized the strategy to FACS-purify the populations and assess gene expression (Figures S3B-S3D). Fitting with a recent report (Jin et al., 2021), washing the liver prior to digestion enriched the peritoneal macs in the wash fraction, demonstrating these were contaminants on the liver surface rather than being present in the liver tissue itself (Figure S3E). While the CITE-seq markers did not discriminate between cluster7 and cluster8, adding CD207 to the panel enabled the non-KCs to be divided into $\mathrm{CD}_{20}{ }^{+}$and CD207- macs (Figure S3F). Fitting with their designation as capsule macs, the relative abundance of $\mathrm{CD} 207^{+}$macs was increased if we dissected and digested the capsule (Figure S3F). However, although Molecular Cartography confirmed the pres-

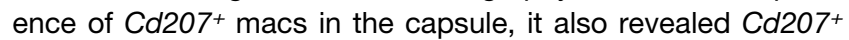
macs at the $\mathrm{CV}$, which were rarely found at the PV (Figures $2 \mathrm{E}-2 \mathrm{H}$ and S3G-S3J). Thus, cluster7 consists of both capsule and CV CD207+ macs. This finding further demonstrates the need for spatial approaches to confirm cell identities. Molecular Cartography also identified macs at the PVs and CVs expressing
Ccr2 and Chil3 (Figures 2G, 2H, S3H, and S3J), resembling transitioning monocytes (cluster11). Finally, a population of Gpnmbexpressing macs were found to be enriched around the bile ducts (Figures 2G, 2H, S3H, S3K, and S3L). As Gpnmb expression is cluster8 specific (Figure $2 \mathrm{~B}$ ), and these cells resemble LAMs (Remmerie et al., 2020), we termed these cells bileduct LAMs.

KCs and LAMs are functionally distinct in the homeostatic murine liver

We next sought to investigate the differences between $\mathrm{KCs}$ and LAMs. Analysis of GO terms associated with biological processes for these cells suggested that KCs may play a role in regulating humoral responses, while LAMs were more broadly associated with immune responses (Figures $2 \mathrm{I}$ and $2 \mathrm{~J}$ ). Consistent with this, in the 100-plex protein microscopy data we noted that a significant proportion of the B cells present were interacting with $\mathrm{KCs}$, which was not observed with T cells (Figure $2 \mathrm{~K}$ ). This suggests cross-talk between these two populations, potentially linked to the high expression of the $\mathrm{B}$ cell chemokine $\mathrm{Cxc} / 13$ by murine KCs (Table S2). To assess the inflammatory nature of LAMs compared with KCs, we FACS-purified the cells and performed qPCR analysis to examine expression of various cytokines. To enable LAM purification, we eliminated the capsule prior to digesting the tissue. Fitting with the GO analysis, LAMs expressed more $/ 11 \mathrm{~b}$ at steady state compared with KCs (Figure 2L). However, despite this, upon in vivo TLR4 stimulation, they were less responsive than $\mathrm{KCs}$, both in terms of pro- and anti-inflammatory cytokines (Figure $2 \mathrm{~L}$ ), possibly indicative of LPS tolerance. This may result from their location at the PV and hence exposure to blood from the intestine, although this remains to be tested. Taken together this highlights the distinct nature and functions of these cells; however, further research is required to determine the precise roles of these cells.

Macrophage subsets reside in distinct spatial niches As all the mac populations are in close contact with CD45- cells in their local environment (Bonnardel et al., 2019; Figure S3M), we further analyzed the CD45- cells, identifying multiple subsets of ECs and SCs and a gating strategy to distinguish them (Figures 3A-3C and S4A-S4C; Table S3). ECs could be further subdivided into 4 distinct clusters and analysis of their locations allowed them to be identified as CV ECs (cluster10), LSECs

Figure 2. A population of macrophages reside around the bile duct in the healthy murine liver (A) UMAP of murine myeloid cells $(71,261$ cells/nuclei) isolated from Figure 1B and re-clustered with TotalVI.

(B and C) Top DEGs (B) and DEPs (C) between cell types.

(D) Expression of Gpnmb and Cd207.

(E) Expression of VSIG4 and F4/80 (left) or MHCII, CD11c, and DAPI (right) by confocal microscopy. Capsule macs identified by white arrows. Scale bars, $50 \mu$ m. (F) Molecular Cartography of indicated genes at liver capsule.

(G) Expression of VSIG4, F4/80, GLUL, and DAPI (left) or F4/80 or CCR2 (right, inset) by confocal microscopy. Scale bars, $100 \mu \mathrm{m}$.

(H) Molecular Cartography of indicated genes at portal triad. PV, portal vein; CV, central vein; HA, hepatic artery; BD, bile duct. Arrows indicate specific cell types, where color corresponds to markers. Images are representative of 2-4 mice.

(I and J) Top GO terms for KCs (I) and bile-duct LAMs (J).

(K) Representative image showing expression of VSIG4 (red) CD19 (yellow) and CD3E (magenta) by MICS analysis (left) and \% of B or T cells found with/without a $\mathrm{KC}$ per field of view (right). Data are pooled from multiple fields of view in 2 mice. ${ }^{\star \star *} \mathrm{p}<0.001$ Student's t test.

(L) Mice (29-week-old) were treated with $3.5 \mathrm{mg} / \mathrm{kg}$ LPS or PBS and $2 \mathrm{~h}$ later, livers were harvested without the capsule. KCs and LAMs were FACS-purified and expression of $/ / 1 b$, Tnf, IL 10, and II18 was examined by GPCR, compared with $b$-actin. ${ }^{*} p<0.05,{ }^{* *} p<0.01,{ }^{* \star *} p<0.001$, ${ }^{* * \star *} p<0.0001$, one-way ANOVA with Bonferroni post-test. See also Figure S3 and Table S2. 
A

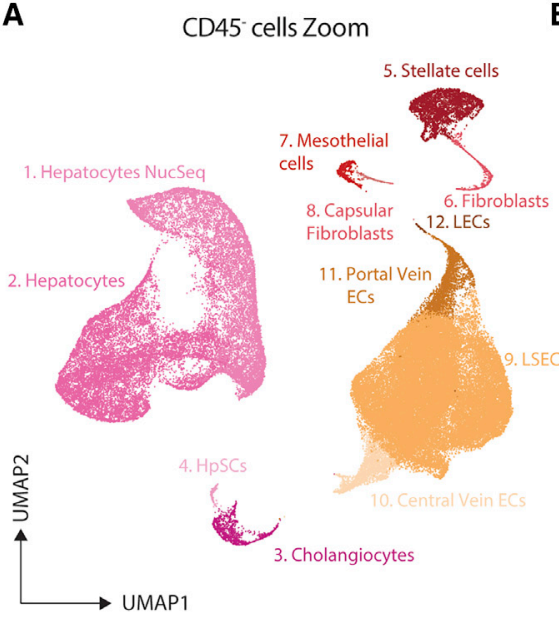

B

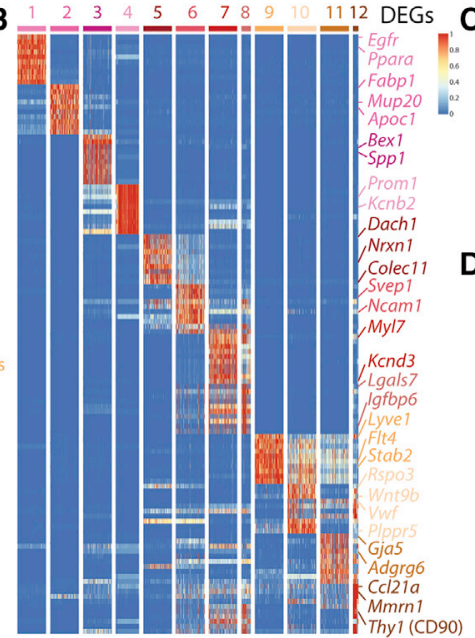

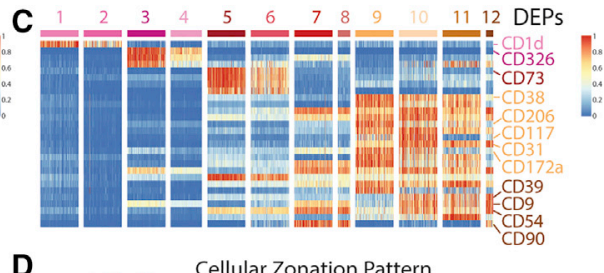

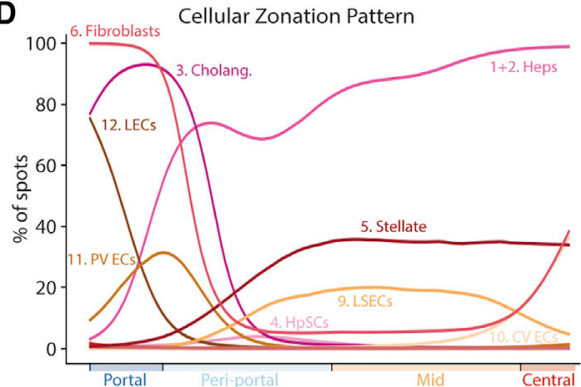

E

Portal Triad Endothelial (mRNA)

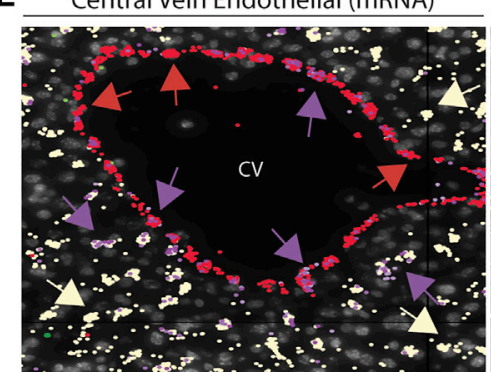

F

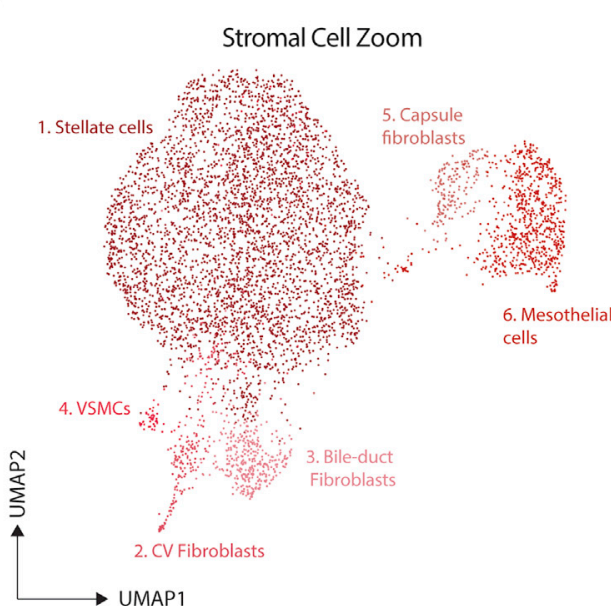

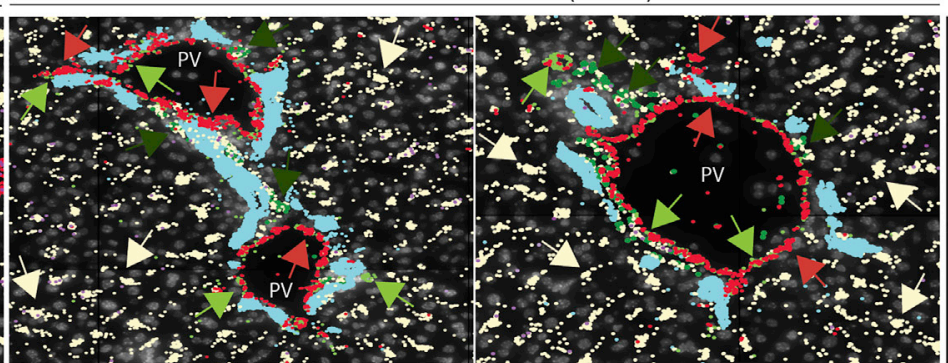

H
EGs

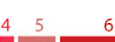

G

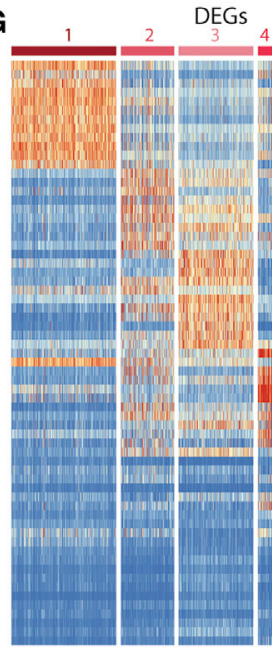

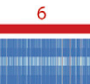

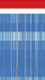

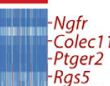
Colect1 $0_{0.2}^{0.4}$
6. Mesothelial Cells

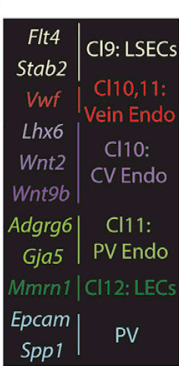

PV

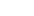

\subsection{5
0.4
0.3
0.2
0.1
0.0}

4. VSMCS

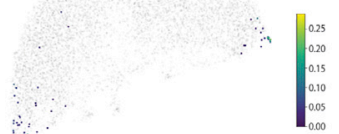

$I_{\text {Mesothelial cells \& Capsule Fibroblasts (mRNA) }}$
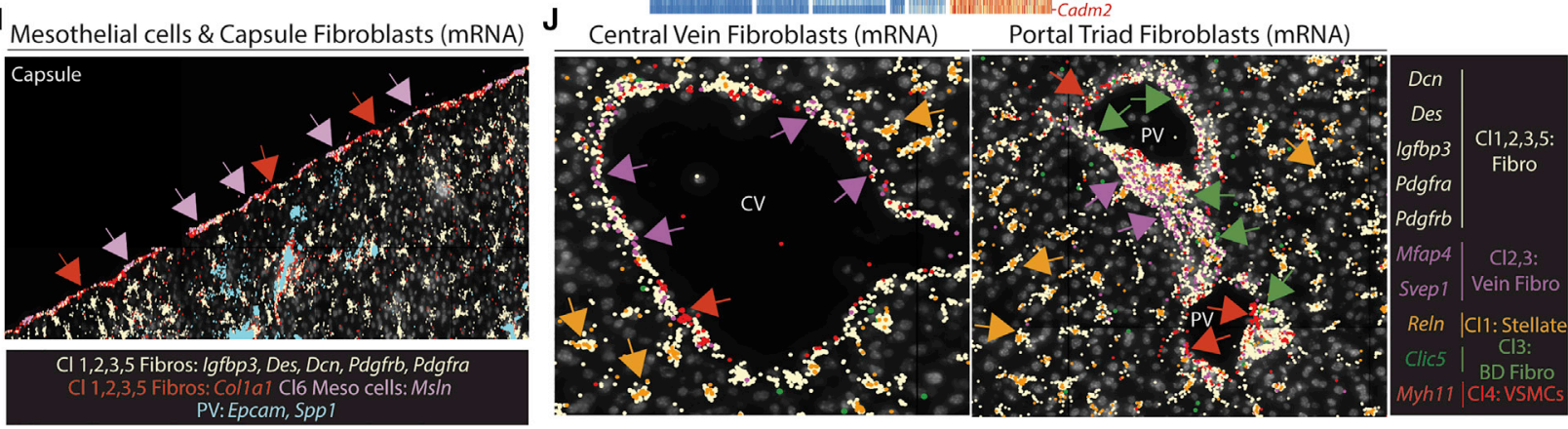
(cluster9), PV ECs (cluster11), and lymphatic ECs (LECs; cluster12) (Figures 3D, 3E, S4D, and S4E). As Visium found fibroblasts at both the PVs and CVs (Figure 3D), and as a previous report has suggested the presence of distinct subsets within these cells (Dobie et al., 2019), we further zoomed in on the SCs to better assess their heterogeneity (Figures 3F, 3G, S4AS4C, and S4F; Table S4). This revealed subsets of mesothelial cells and fibroblasts restricted to the capsule (Figures $3 \mathrm{H}$ and 3I). Myh11+ vascular smooth muscle cells (VSMCs) were localized around hepatic arteries, PVs and CVs (Figures $3 \mathrm{~F}-3 \mathrm{H}, 3 \mathrm{~J}$, and S4G) and Mfap4 ${ }^{+}$Svep $1^{+}$Clic5 ${ }^{-}$Reln ${ }^{-}$fibroblasts were found to be CV fibroblasts (Figures 3G, 3H, 3J, and S4G). Finally, we identified a subset of Clic5 ${ }^{+}$Reln ${ }^{+}$fibroblasts (cluster3), which were localized around the cholangiocytes, that we termed bileduct fibroblasts (Figures 3J and S4G). Taken together, the presence of these spatially distinct subsets of ECs and SCs highlights the uniqueness of the specific microenvironments in which the distinct mac populations reside.

\section{An in silico gating strategy for murine hepatic} lymphoid cells

Finally, in addition to providing gating strategies for myeloid and CD45- cells, we also wanted to investigate if the CITE-seq data would allow us to develop similar strategies for the lymphoid populations. To examine this, we re-clustered the T cells, NK cells, ILCs, B cells, and pDCs from Figure 1B identifying 12 distinct populations (Figure S5A). Analysis of the DEGs highlighted that while B cells, NK cells, ILC1s, and pDCs were distinct populations, there was considerable overlap between the transcriptomic profiles of the T cells (Figure S5B; Table S5). However, by analyzing the DEPs we were able to define distinct subsets including naive CD4 and CD8 T cells, $T_{\text {Regs }}, T_{H} 1 s, C T L s$, and $\mathrm{T}_{\mathrm{H}}$ 17s (Figures S5A and S5C; Table S5). Moreover, we were able to design a gating strategy to isolate the distinct populations (Figure S5D).

\section{A practical proteogenomic atlas of the healthy} human liver

To determine the degree of conservation between the mac subsets and their different microenvironmental niches between the mouse and the human liver, we next generated a proteogenomic atlas of the human liver using sc/snRNA-seq and CITE-seq on 19 liver biopsies (Figures 4A, 4B, S6A, and S6B; Tables S1 and S5). Of these, most were histologically healthy with only 5 patients showing $>10 \%$ hepatic steatosis in the absence of any significant fibrosis (Table S6). Cellular proportions varied according to the isolation technique used, and while there was some variability between patients, this was not linked to the surgery (Figures S6C$\mathrm{S6E})$. Further confirming the lack of fibrosis in the steatotic livers, we did not detect any increase in CTLs (Figures S5E-S5I; Table S5), which have been shown to correlate with non-alcoholic steatohepatitis (NASH) (Haas et al., 2019). A significant increase in CTLs was detected in the setting of murine NASH induced by feeding a western diet (WD) for up to 36 weeks, demonstrating that this is not due a limitation in detecting these differences using CITE-seq (Figures S5J-S5M; Table S7). As Visium reliably located murine hepatic cells, we used this to locate the cells of the human liver in 4 biopsies (Figure 4C). However, the Visium spots from patients with $>10 \%$ steatosis were found to cluster separately from the healthy samples $(<10 \%$ steatosis; Figures $4 \mathrm{D}$ and $4 \mathrm{E})$. We therefore used the healthy samples to calculate a baseline zonation and then transferred this trajectory onto the steatotic samples (Figures $4 \mathrm{~F}$ and S6F). This identified the steatosis to be predominantly present in regions expressing peri-central zonation genes like CYP2E1. This zonation pattern was further validated using Molecular Cartography (Figure S6G). This fits with previous clinical studies demonstrating peri-central steatosis to be most common in non-alcoholic fatty liver disease (NAFLD) patients, especially in early disease (Chalasani et al., 2008; Kleiner and Makhlouf, 2016). However, as these peri-central regions are larger than in the healthy controls, it could also imply that the presence of steatosis alters expression of the zonated hepatocyte genes, but this remains to be tested. Notably, the overall cellular distribution was not impacted by the presence of steatosis, although neutrophils and monocytes and monocytederived cells were preferentially localized peri-centrally in the steatotic patients correlating with the presence of steatosis (Figure 4G). MICS 100-plex protein analysis further validated the cellular distributions predicted by Visium and confirmed the increase in neutrophils in the steatotic livers (Figures S6H and S6I).

\section{Evolutionarily conserved transcriptomic and proteomic identity of KCs}

To date, no validated markers of bona fide human KCs have been described. Explaining the difficulty to accurately define human $\mathrm{KCs}$, we found monocytes and macs formed a single continuum in the human sc/snRNA-seq data, preventing a simple definition of human KCs (Figure 4B). Notably, a similar continuum from monocyte to $\mathrm{KCs}$ was also observed in the NASH murine liver (Figures S5J and S6J-S6N, Table S8). Consistent with our previous report (Remmerie et al., 2020), we observed both long-term resident Timd4-expressing KCs and recently recruited Timd4-

Figure 3. Hepatic macrophage populations reside in distinct niches

(A) UMAP of murine CD45 ${ }^{-}$cells $(83,410$ cells/nuclei) isolated from Figure $1 \mathrm{~B}$ and re-clustered with TotalVI.

(B and C) Top DEGs (B) and DEPs (C) between cell types.

(D) Indicated cell signatures from sc/snRNA-seq mapped onto the Visium zonation data.

(E) Molecular Cartography of indicated genes at central vein (left) and 2 different portal triads (center and right).

(F) UMAP of murine stromal cells (5,430 cells/nuclei) isolated from the UMAP in Figure $3 \mathrm{~A}$ and re-clustered with scVI.

(G) Top DEGs between different cell types identified.

$(\mathrm{H})$ Identification of mesothelial cell (top) and VSMC (bottom) signatures on zonated Visium data.

(I and J) Molecular Cartography of indicated genes at the liver capsule (I) or the central vein (J; left) and portal triad (J; right). PV, portal vein; CV, central vein; HA, hepatic artery; BD, bile duct. Arrows indicate specific cell types, where color corresponds to markers. Images are representative of $2-4$ mice. See also Figure S4 and Tables S3 and S4. 
A

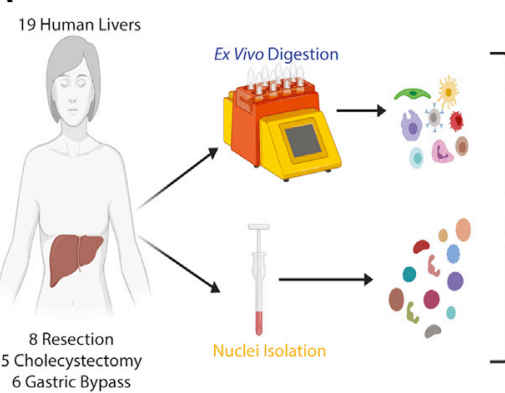

B Human Liver (pool of techniques and patients)
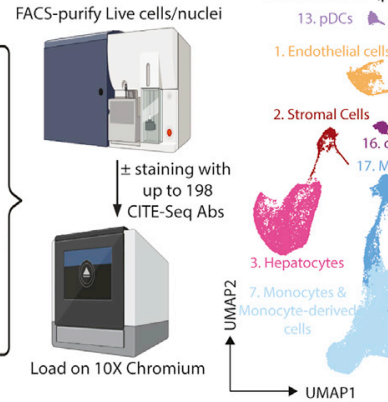

C Visium (pool of patients)
E

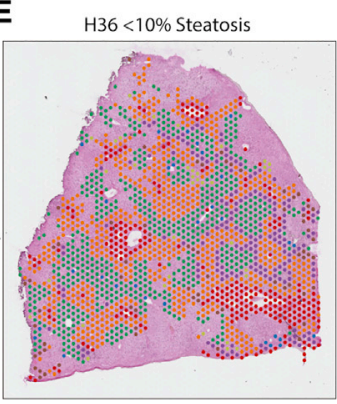

H
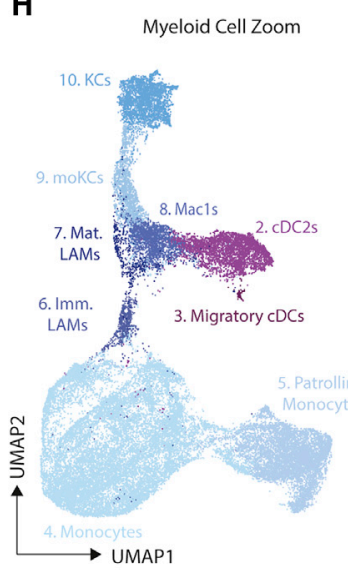

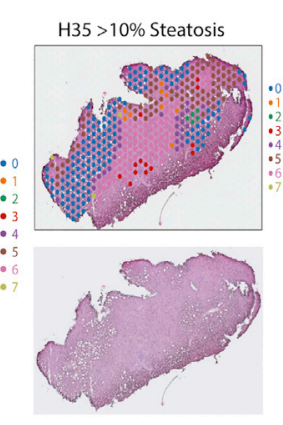

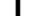

$\mathbf{F}$

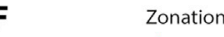

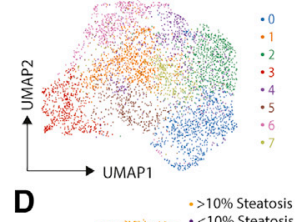

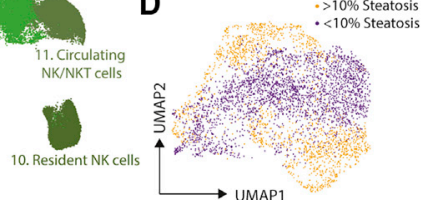

G Cellular Zonation Pattern $<10 \%$ Steatosis
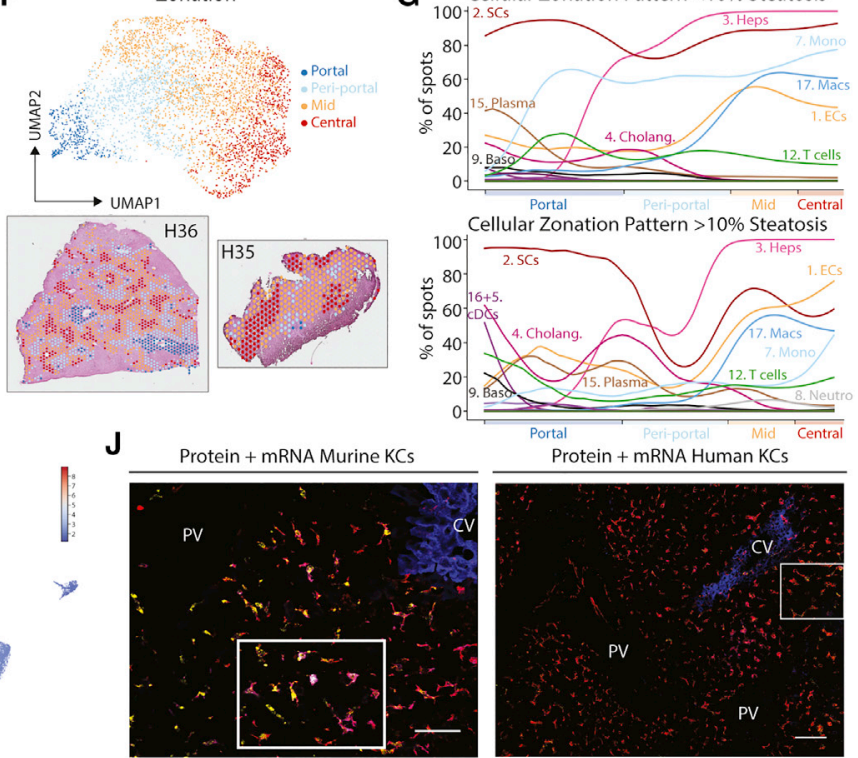

Protein + mRNA Human KCs

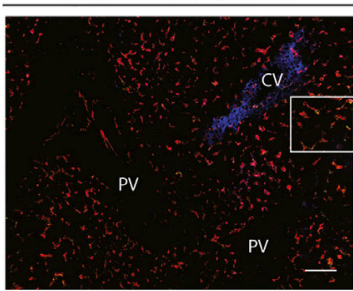

$y^{. c D C 15}$
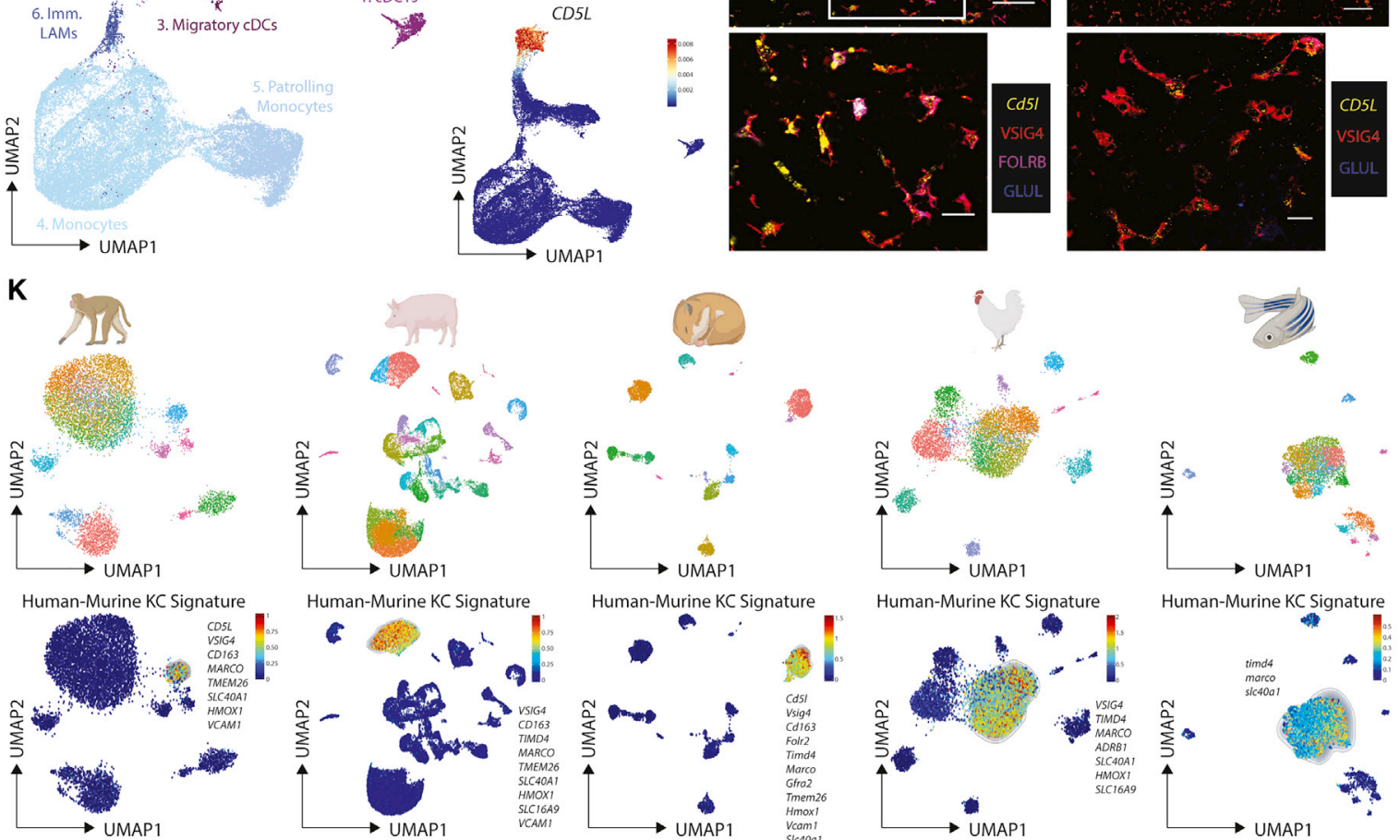

Human-Murine KC Signature
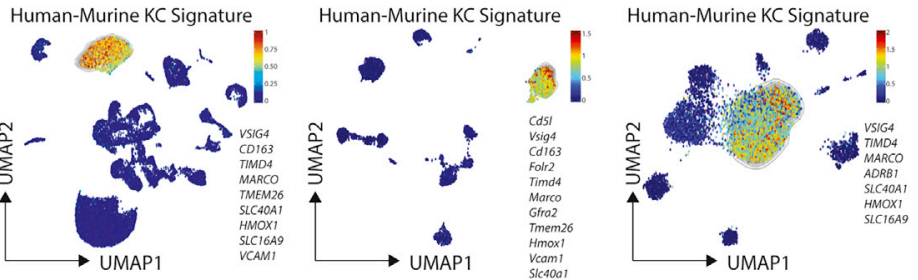

Human-Murine KC Signature

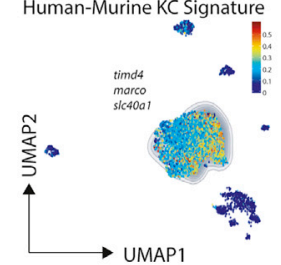

(legend on next page) 
monocyte-derived KCs (moKCs; Figures S6J-S6N). As the presence of such a continuum in the human liver suggests that there may also be monocyte contribution to the $\mathrm{KC}$ pool in the healthy human liver, we next zoomed in on myeloid cells to examine this, identifying 10 clusters (Figures 4H, S6O, and S6P; Table S2). To define the $\mathrm{KCs}$, we examined expression of the top 25 murine $\mathrm{KC}$ genes by these clusters, which identified cluster 10 to be the genuine human KCs (Figure S6Q). Unlike in mice, these were preferentially located in the mid zone (Figure S6R). Cluster9 also expressed many of these genes but lacked TIMD4 (Figure $\mathrm{S} 6 \mathrm{Q}$ ), suggesting that these cells may be recently recruited moKCs. The presence of moKCs in the liver is consistent with reports that host-derived macs are identified in transplanted donor livers (Bittmann et al., 2003; Pallett et al., 2020) and suggests that the $\mathrm{KC}$ population may be a mix of embryonic and monocytederived cells. Although not the case at mRNA level, VSIG4 was found to be the best human KC protein marker in the CITE-seq data, while FOLR2, CD163, and CD169 were also identified as useful markers of these cells for flow cytometry and confocal microscopy on frozen and paraffin sections (Figures $4 \mathrm{I}$ and S6SS6X). Co-staining human livers for VSIG4 protein and KC-specific CD5L mRNA and MICS 100-plex protein analysis also confirmed the mid-zonal localization of KCs (Figures 4J and S6U). To assess if $\mathrm{KC}$ identity was further conserved in evolution, we profiled macaque, pig, hamster, chicken, and zebrafish livers (Figure 4K). We identified the $\mathrm{KCs}$ in an unbiased manner by mapping the conserved human-mouse KC signature onto the datasets (Figures $4 \mathrm{~K}$ and S7A-S7C). We then examined the main features of each KC population identified (Figures S7D-S7H; Table S9). A strong overlap in transcriptomes across species was observed likely due to the conserved expression of core KC transcription factors (Figure S7I). However, each species also harbored a number of unique KC genes (Figure S7J; Table S9). VSIG4 protein expression was also conserved in pig and macaque KCs (Figures S7K-S7M). Similarly, we were also able to identify most of the other hepatic cells across species on the basis of conserved genes (Figures S7N and S7O). cDC2s were the main exception to this, as specific cDC2 marker genes were not conserved across all species (Figure S7O).
LAM location is altered in the steatotic liver

Alongside KCs, we also identified distinct clusters of macs in the human myeloid cells (Figure $4 \mathrm{H}$ ). To better understand the nature of these clusters we performed confocal microscopy to examine the specific locations of CD68+VSIG4- macs in the human liver (Figure S8A). This identified CD68 ${ }^{+} \mathrm{VSIG} 4^{-}$macs in the liver capsule, in close proximity to central and PVs as well as at bile ducts (BDs) (Figures 5A-5C and S8A). Similar populations were also observed at the PVs and CVs and at the BDs in the healthy macaque liver (Figure S7M). Examination of the scRNA-seq data and comparison with murine signatures identified immature and mature LAMs, with immature LAMs expressing some monocyte genes (Figures 4H, 5D, S8B, and S8C). Although recently suggested to be specific to fibrotic human livers (Ramachandran et al., 2019), we identified LAMs in all patients profiled with scRNA-seq, but there was a trend toward increased proportions of LAMs in the livers with $>10 \%$ steatosis (Figure S8D) consistent with the increased population of LAMs in murine NAFLD (Figures S6J-S6N). As in the healthy mouse, Visium identified human LAMs in portal zones of non-steatotic livers. However, in steatotic human livers, LAMs were primarily located peri-centrally, in zones with steatosis (Figure 5E), suggesting that monocytes are recruited to distinct locations in the healthy and obese liver where they then differentiate into LAMs. This altered location of LAMs was further validated by confocal microscopy and Molecular Cartography (Figures $5 \mathrm{~F}-5 \mathrm{H}$ ). However, this analysis did not identify any capsule macs, suggesting that these cells may be absent from our UMAP, likely as a result of the small amount of capsule tissue on a biopsy. The Mac1 population expressing IGSF21 was present in very low numbers throughput the tissue (Figures $5 \mathrm{G}$ and $5 \mathrm{H}$ ). This coupled with their similar transcriptomic profile to moKCs could suggest that these are moKC precursors as observed in the mouse, but this requires further study. Focusing on the LAMs, the change in their location in the steatotic human liver was also observed in the murine NAFLD model. Here, LAMs were found across portal, periportal, and mid zones (Figures 5I, S8E, and S8F), fitting with the presence of steatosis in these regions and consistent with our previous report (Remmerie et al., 2020). Comparison of DEGs between LAMs in

Figure 4. Identification of bona fide Kupffer cells across species

(A) Cells/nuclei were isolated from liver biopsies ( 1-2 $\mathrm{mm}^{3} ; 14$ cells, 5 nuclei) from patients undergoing either liver resection, cholecystectomy or gastric bypass. Live cells/intact nuclei were FACS-purified. Either total live, live CD45 ${ }^{+}$, and live CD $45^{-}$or live CD45 ${ }^{+}, \mathrm{CD}^{-}$, and CD19- cells were sorted. 7 cell samples were stained with a panel of 198 barcode-labeled antibodies for CITE-seq analysis. All datasets were pooled together and after QC, 167,598 cells/nuclei were analyzed using TotalVI.

(B) UMAP of sc/snRNA-seq data.

(C) UMAP of Visium data from 4 patient biopsy samples.

(D) Split of Visium spots based on \% steatosis.

(E) Healthy and steatotic Visium liver tissue with clusters overlaid and $\mathrm{H}+\mathrm{E}$ staining to identify steatotic zones.

(F) Zonation of Visium data (top) with zonation pattern mapped onto liver tissue (bottom).

(G) Indicated cell signatures from sc/snRNA-seq mapped onto Visium zonation trajectory, healthy (top), steatotic (bottom).

(H) Myeloid cells $(40,821$ cells) were isolated from Figure 4B and re-clustered with TotalVI.

(I) Expression of VSIG4 protein (top) and CD5L mRNA (bottom).

(J) Expression of VSIG4, F4/80, FOLRB, and GLUL combined with Cd5I/CD5L on murine (left) and human (H25; right) livers. Scale bars, $50 \mu \mathrm{m}$. Inset in bottom panels. Scale bars, $20 \mu \mathrm{m}$. Images are representative of $2-4$ livers.

(K) Livers (2/species) were isolated from healthy macaque, pig, chicken, hamster, and zebrafish. Cells were isolated by ex vivo digestion for CITE-seq (pig; 198 human antibodies) or scRNA-seq (hamster, chicken, and zebrafish), or nuclei were isolated for snRNA-seq (macaque). Total live cells (hamster, chicken, and pig), DsRed $^{+}$GFP $^{+}$cells (zebrafish) or nuclei (macaque) were FACS-purified. Following QC, 8,483 nuclei (macaque) or 21,907 (pig), 5,965 (hamster), 7,457 (chicken), and 4,957 (zebrafish) cells were analyzed using TotalVI (pig) or scVI (macaque, hamster, chicken, and zebrafish) (top). KCs were identified using the humanmurine KC signature and the signature finder algorithm (Pont et al., 2019) (bottom). See also Figures S6 and S7 and Tables S1, S2, S3, S5, S6, S8, and S9. 
A

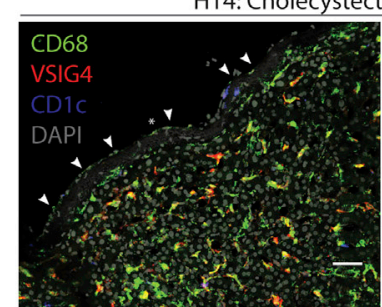

D

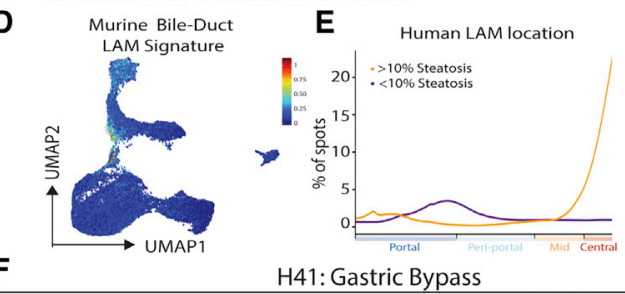

B

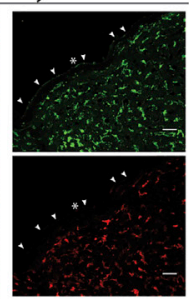

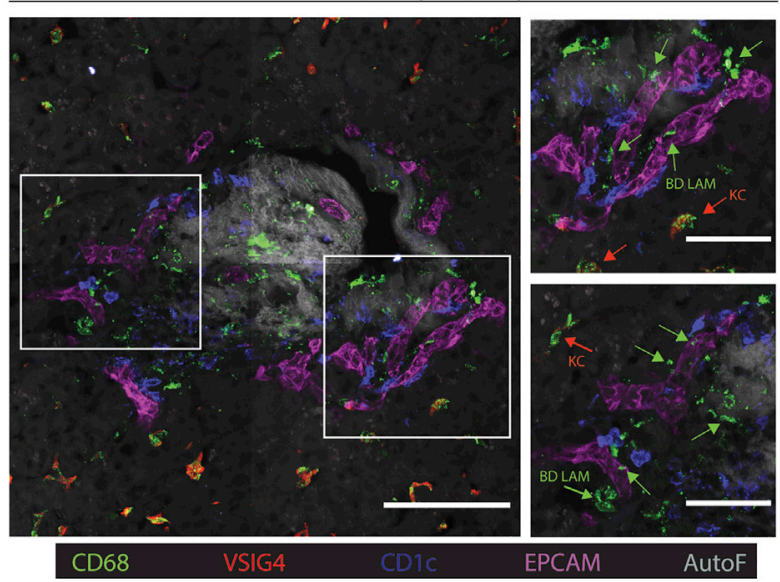

C

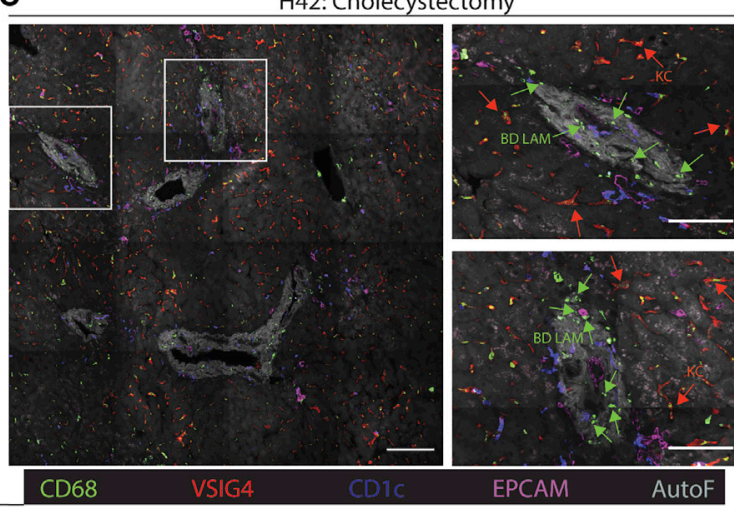

H Macrophage Subsets Steatotic Human Liver - H35

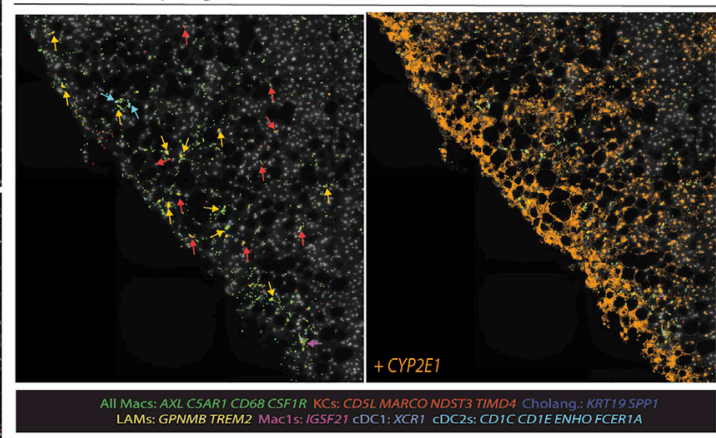

LAM DEGS SD vs WD

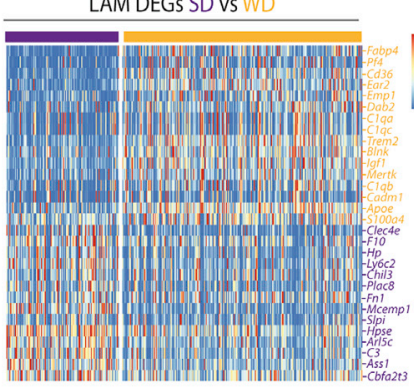

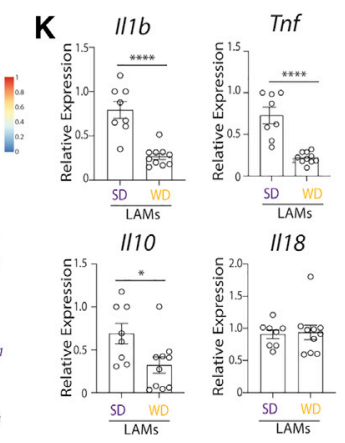


standard diet (SD) and WD-fed mice identified that LAMs had a more mature phenotype in WD-fed mice, downregulating their expression of some monocyte genes and increasing their expression of prototypical mac markers, consistent with the presence of both immature and mature LAMs in the human liver (Figure 5J). Fitting with a more mature phenotype, WD-derived LAMs also expressed lower levels of $/ / 1 \mathrm{~b}, \mathrm{Tnf}$, and $/ / 10$ compared with SD LAMs (Figure 5K). While further studies are required to assess the precise functions of these cells in NAFLD, this could further suggest a protective rather than a pathogenic role for LAMs (Daemen et al., 2021).

Potential role for CD45- hepatic cells in determining macrophage localization

Given the altered localization of KCs in the healthy murine versus human liver and LAMs in the healthy versus steatotic liver of mice and humans, we next sought to investigate how the cells of the mac niches differed in these settings. Analysis of CD45- cells in the human liver identified similar (sub)populations of ECs, SCs, and hepatocytes as observed in the healthy mouse liver (Figures 6A and 6B; Table S3). However, while the 100-plex MICS analysis detected a second subset of portal fibroblasts expressing DESMIN (Figure S6H), we did not detect these cells in our UMAP, likely due to difficulties detecting DESMIN with snRNA-seq. No significant differences were found in terms of localization of the identified $\mathrm{CD} 45^{-}$cells that could explain the altered location of KCs compared with the mouse (Figures 6C and 6D). With this in mind, we next utilized NicheNet to examine potential ligand-receptor pairs between KCs and CD45- cells present only in the human that could regulate $\mathrm{KC}$ location. This analysis identified CCL23 and CCL14 expression by LSECs and CCL16 by hepatocytes that binds to CCR1 expressed by human KCs but not murine KCs (Figure 6E). Crucially, these ligands were preferentially located peri-centrally (Figure 6F) and thus represent interesting targets for further study regarding the potential regulation of $\mathrm{KC}$ location in the human liver.

We next aimed to investigate the role of CD45- cells in the regulation of LAM location in the healthy versus steatotic liver. Given the low number of steatotic human samples in our sc/ snRNA-seq analysis and the variation between patients, we turned to the murine NASH model to investigate this. Zooming in on SCs (Figure 6G; Table S10), we identified that there was an increase in fibroblasts in the WD-fed mice. There was also a considerable overlap between fibroblasts and HSCs in terms of their gene expression profiles suggesting there may be a differentiation trajectory between the HSCs and the fibroblasts, although this remains to be validated in vivo. We also noted a sub-population of fibroblasts expressing $\mathrm{Cc} / 2$, a known ligand for CCR2 which is expressed on monocytes recruited to the liver during NAFLD (Remmerie et al., 2020), as well as Cd44 and Vcam1, two genes involved in monocyte recruitment and adhesion (Johnson and Ruffell, 2009; Meerschaert and Furie, 1995). Both fibroblast populations were enriched in the periportal steatotic regions (Figure 6l), in which we also observe enrichment of the LAMs (Figure 5I). We also found high CCL2 and Cd44 expression in human fibroblasts (Table S3), potentially recruiting bileduct LAMs. Together, this suggests that fibroblasts may play an important role in recruiting LAMs.

\section{Differential NicheNet analysis across species reveals a} crucial role for the ALK1-BMP9/10 axis in KC development Having identified a potential role for the mac niche cells in regulating mac subset location, we next sought to determine the involvement of these cells in regulating mac phenotypes. To assess the roles of conserved cell-cell interactions in driving mac heterogeneity across species, we performed a differential NicheNet (Browaeys et al., 2019) analysis between the distinct hepatic macs and the CD45- cells present in their respective niches focusing on ligands and receptors conserved in both human and mouse. This revealed very few specific ligand-receptor pairs for LAMs (Figure S8G; data not shown), hinting that local factors such as metabolites rather than unique cell-cell interactions may drive the LAM phenotype. Indeed, this would be consistent with the presence of LAM-like cells in multiple tissues including the obese adipose tissue, the brain, the lung, and the heart (Jaitin et al., 2019; Keren-Shaul et al., 2017; Liao et al., 2020; Rizzo et al., 2020). In line with this, BM monocytes cultured with acetylated low-density lipoprotein expressed LAM-associated genes (Figure $7 \mathrm{~A}$ ), demonstrating a dominant role for lipids in inducing the LAM phenotype. Conversely, for $\mathrm{KCs}$, we found multiple ligandreceptor pairs to be conserved between human and mouse (Figures $7 \mathrm{~B}$ and $\mathrm{S} 8 \mathrm{G})$. One of these, an activin receptor-like kinase (ALK1)-bone morphogenic protein (BMP) $9 / 10$ circuit between KCs (ALK1; encoded by Acvrl1) and stellate cells (BMP)9/10 encoded by $G d f 2 / B m p 10$ respectively) was found to be conserved

Figure 5. LAMs are found at the bile duct in the healthy liver, but at zones of steatosis in the obese liver

(A) Expression of indicated markers at the capsule of the healthy human liver $(\mathrm{H} 14)$ by confocal microscopy. Scale bars, $20 \mu \mathrm{m}$. Arrowheads indicate capsule macs.

(B and C) Representative images showing expression of indicated markers at the portal triad by confocal microscopy. Insets on right. Scale bars, $100 \mu \mathrm{m}$. Insets scale bars, $50 \mu \mathrm{m}$ (B). Scale bars, $150 \mu \mathrm{m}$. Insets scale bars, $75 \mu \mathrm{m}$ (C).

(D) Human bile-duct LAMs identified using the murine bile-duct LAM gene signature and the signature finder algorithm (Pont et al., 2019).

(E) Human LAM signature from scRNA-seq mapped onto the Visium zonation data, healthy (purple), steatotic (yellow).

(F) Expression of indicated markers by confocal microscopy. Insets on right. Scale bars, $150 \mu \mathrm{m}$. Insets scale bars, $75 \mu \mathrm{m}$.

$(\mathrm{G}$ and $\mathrm{H})$ Expression of indicated genes in healthy $(\mathrm{G})$ and steatotic $(\mathrm{H})$ human liver. Insets on right. Images are representative of 2 patients per condition.

(I) Mice were fed a western diet (WD) or standard diet (SD) for 36 weeks to induce NAFLD and Visium analysis was performed. Analysis is pooled from 1 liver slice from the SD condition and 3 liver slices from the WD condition. Zonation pattern and H\&E staining (left) and LAM and KC location (right).

(J) Heatmap showing DEGs between LAMs from SD (purple) and WD (yellow) fed mice (24 + 36 weeks pooled).

(K) LAMs were FACS-purified from the liver of mice fed the SD or WD for 24 weeks (with removal of capsule prior to digestion), RNA was isolated and expression of indicated genes was assessed by qPCR relative to $\beta$-actin. ${ }^{\star} p<0.05,{ }^{\star \star \star \star} p<0.0001$, Student's $t$ test. Data are pooled from 2 independent experiments with $n=7-$ 10 mice per group. Images from (A-C and F) are representative of 4-5 patients per condition. PV, portal vein; CV, central vein. See also Figure S8 and Table S2. 
A

Human CD45-cells Zoom
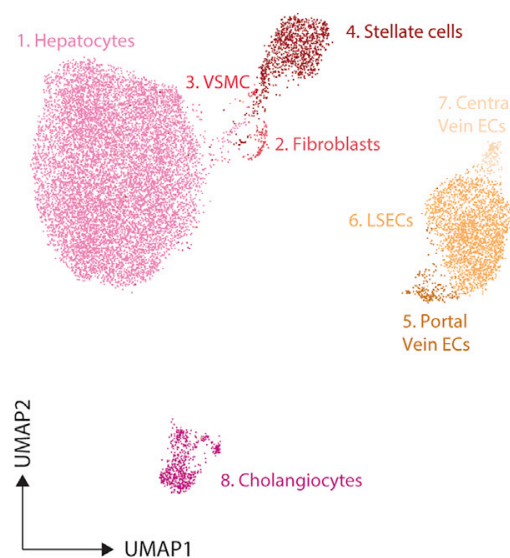

B

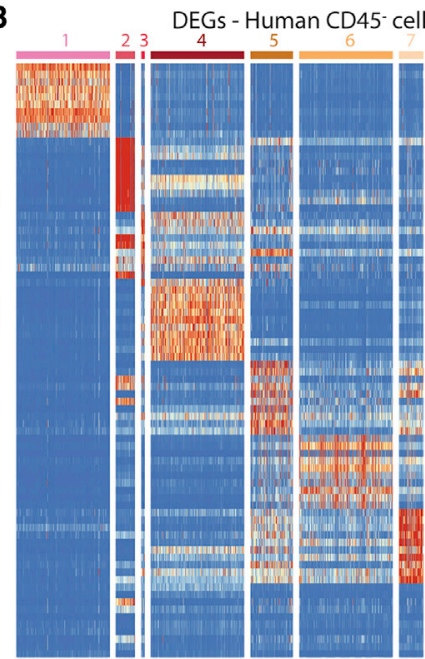

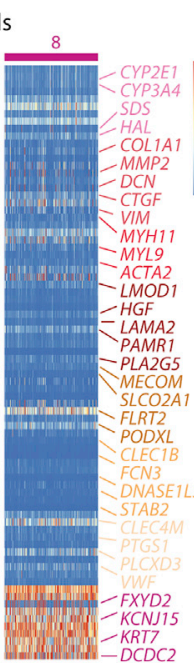

C

Cellular Zonation Pattern $<10 \%$ Steatosis
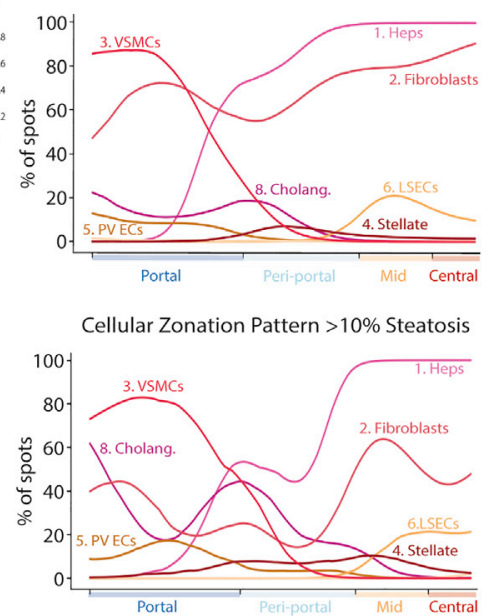

D CD45-Cell Subsets Healthy Human Liver - H36

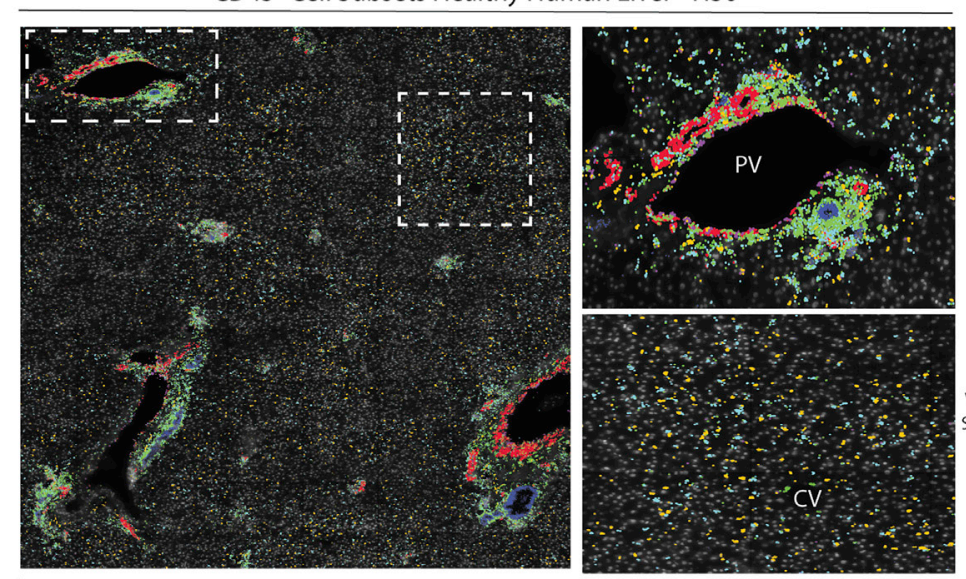

E NicheNet: Human KC specific Ligand-Receptor Pairs

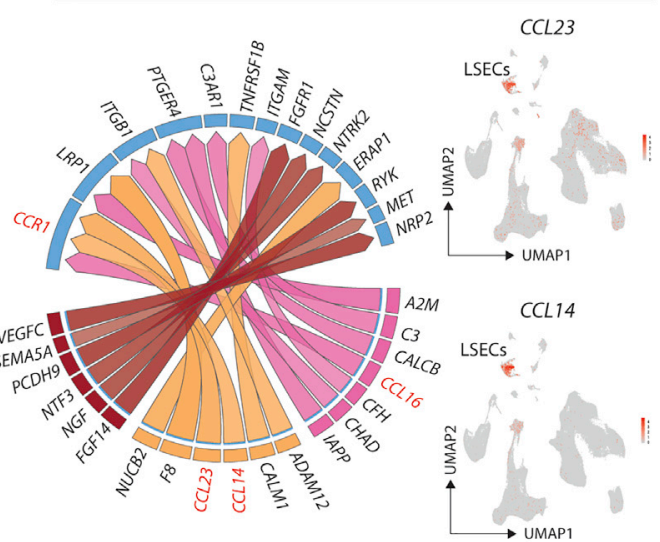

Fibroblasts: COL1A1 MMP2 DCN MFAP4 VSMCS MYH11 Cholang: KRT19 EP
LSECS: STAB2 FLT4 LYVE1 Vein Endo: VWF Stellate: COLEC11 HGF

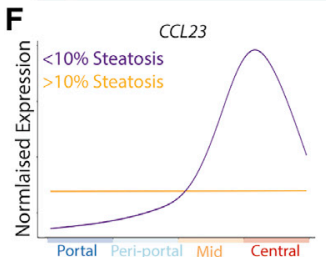

CCL14

CCL16
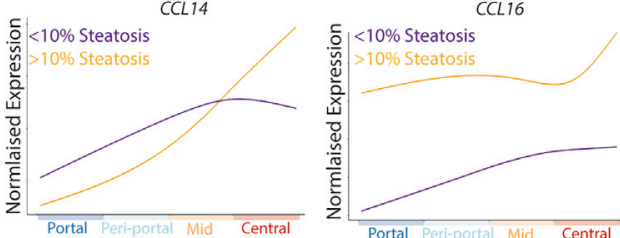

CCR1 Human

CCR1 Mouse SD \& WD

CCL16

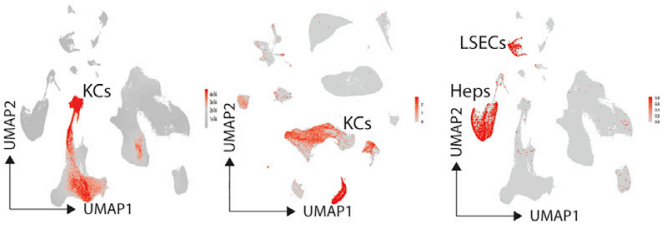

G

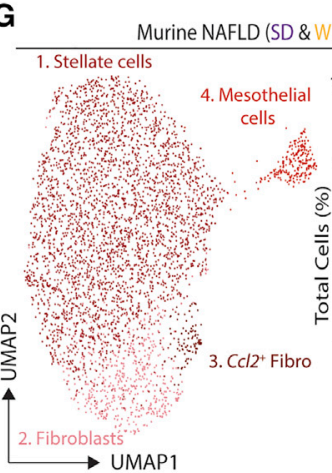

H DEGs - Mouse NAFLD (SD \& WD) Stromal cells

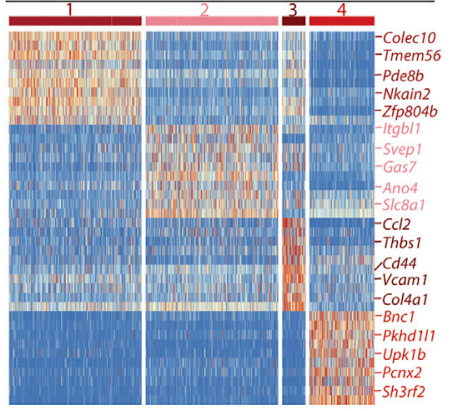

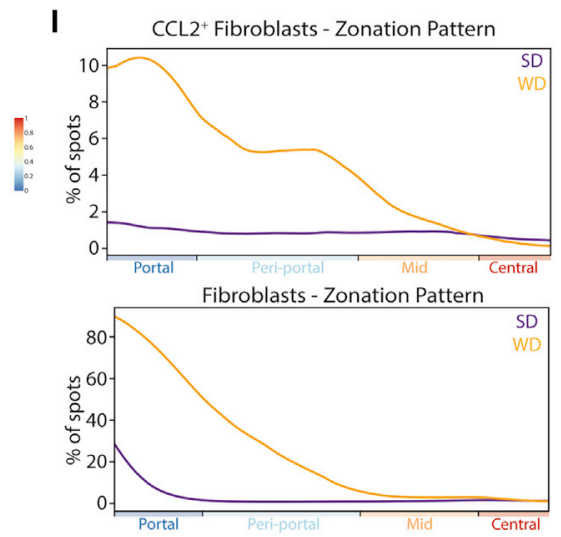


in all 7 species and was predicted to control the expression of a number of the conserved $\mathrm{KC}$ genes (Figures $7 \mathrm{~B}, 7 \mathrm{C}$, and $\mathrm{S} 8 \mathrm{H}$ ). To validate a role for this axis in KCs, we generated Fcgr1-Cre $\times$ Acvrl1 $1^{\mathrm{f} / \mathrm{fl}}$ mice, eliminating ALK1 specifically from CD64-expressing macs (Scott et al., 2018). This led to an almost complete loss of VSIG4+ $\mathrm{KCs}$ (Figures 8D-8F), demonstrating that evolutionarily conserved ALK1 signaling is crucial for KCs. To determine if ALK1 is required for KC maintenance we generated Clec4f-Cre $\times A c v r l 1^{\mathrm{fl} / \mathrm{fl}}$ mice, eliminating ALK1 only from differentiated KCs (Scott et al., 2018). This revealed a relatively similar phenotype than observed in the Fcgr1-Cre mice, suggesting that ALK1 is also required for KC maintenance (Figure S8I). To directly test the need for ALK1 in KC development, we generated BM chimeras whereby CD45.1 Clec4f-Dtr mice were irradiated with their livers shielded to avoid any radio damage. These mice were then reconstituted with either CD45.2 Acvrl1//fl or Fcgr1CrexAcvrl1 $1^{\mathrm{fl} / \mathrm{fl}}$ BM. 4 weeks later, mice were given a single i.p. injection of DT to deplete the KCs and 7 or 13 days thereafter chimerism within the $\mathrm{KC}$ population was determined (Figure $7 \mathrm{G}$ ). We observed that, already by day $7, \mathrm{KO} \mathrm{BM}$-derived cells were almost completely outcompeted by WT BM-derived cells within the KC pool. This was not observed in monocytes but a similar pattern was observed in VSIG4- macs demonstrating that ALK1 is critically required for early KC development (Figure $7 \mathrm{H}$ ). Finally, while NicheNet predicts that BMP9/10 from stellate cells would signal through ALK1 to induce KC development and maintenance, a prediction in line with our previous NicheNet analysis (Bonnardel et al., 2019), another recent study has suggested that transforming growth factor (TGF) $\beta$ signaling would be important for KCs (Sakai et al., 2019). This prediction was made on the basis of SMAD4 signaling, which is a common downstream effector of both TGF- $\beta$ Receptor and ALK1-induced signaling. As TGF- $\beta$ was also found to participate in an ALK1-TGF- $\beta$ receptor containing signaling complex (Goumans et al., 2003), we thus examined whether TGF- $\beta$ signaling is important for KCs. To this end we utilized an ALK1-Fc trap or TGF- $\beta$ type II receptor (TGF $\beta$ RII)-Fc trap alongside appropriate isotype controls. ALK1-Fc selectively sequesters BMP9/10 (Desroches-Castan et al., 2021), while TGF $\beta$ RII-Fc selectively interferes with TGF$\beta 1 /$ TGF- $\beta 3$ receptor binding (Komesli et al., 2017). Clec4f-Dtr mice were depleted of KCs and simultaneously treated with either receptor-Fc traps or isotype controls and $\mathrm{KC}$ development was examined 7 days later (Figure $7 \mathrm{I}$ ). In line with the chimera study, treatment with ALK1-Fc significantly abrogated KC development; however, blocking TGF $\beta$ signaling had only a minor effect on the proportion of VSIG4- macs (Figure 7J). Altogether this validates the NicheNet prediction and demonstrates that the evolutionarily conserved ALK1-BMP9/10 axis is crucial for the development and maintenance of KCs.

\section{DISCUSSION}

To generate a practical cellular atlas of any human tissue and unravel the cell-cell circuits essential for the identities of cells inhabiting that tissue, four key pieces of information are required: (1) an inventory of all cells present, (2) the location of the different cells within the tissue to identify interactions between neighboring cells, (3) an alignment between the human and animal models allowing for any predicted cell-cell interactions to be perturbed, and (4) the identification of reliable antibody-based panels for the efficient screening of different patients and/or transgenic animals. Here, by integrating single-cell and spatial transcriptomic and proteomic data, we provide these 4 pieces of information for the liver and uncover evolutionarily conserved microenvironmental circuits controlling the development of hepatic macs.

Unraveling the spatial localization of all hepatic cells, we identify LAMs around the bile ducts in the healthy mouse, human, and macaque liver. However, when steatosis is present, LAMs are preferentially recruited to the steatotic regions of the liver. This spatial information at least partially invalidates the hypothesis that LAM identity is specifically induced by fibrotic SCs (Ramachandran et al., 2019). Rather, our data suggest that LAMs are induced by local lipid exposure. We also provide an alignment of the liver atlas across seven species. This reveals the conserved and unique transcriptomic programs of steady-state KCs and uncovers the spatially restricted and conserved ligand-receptor pairs between KCs and the cells constituting their niche. Underlining the need to first characterize the healthy tissue before attempting to understand how disease perturbs the cells, we identify the DLL-NOTCH interaction to be an evolutionarily conserved cross-talk between homeostatic LSECs and KCs and therefore not unique to hepatocellular carcinoma or fibrosis, as proposed (Ramachandran et al., 2019; Sharma et al., 2020). Similarly, we find that FOLR2 expression is not specific to tumor-associated hepatic macs (Sharma et al., 2020) but is expressed by KCs in the healthy mouse and human liver. Finally, we apply a proteogenomic pipeline starting from broad oligo-conjugated antibody panels for both single-cell and spatial profiling. This is crucial as transcriptomic profiling does not always correspond with the ability to detect proteins by flow cytometry or microscopy. By

Figure 6. Macrophage niche cells may regulate macrophage locations in healthy versus steatotic liver

(A) UMAP of human CD45- cells (15,481 cells/nuclei) isolated from Figure 4B and re-clustered with scVI.

(B) Top DEGs between cell types identified.

(C) Indicated cell signatures from sc/snRNA-seq mapped onto the Visium zonation data, healthy (top), steatotic (bottom).

(D) Molecular Cartography localizing distinct CD45- cells.

(E) Circos plot showing NicheNet predicted ligand-receptor pairs between KCs and LSECs, HSCs and hepatocytes uniquely expressed in the human liver (left) and UMAPs showing normalized expression of indicated chemokines in the human liver (right) and CCR1/Ccr1 in the human and murine NAFLD liver (bottom). (F) Zonation of indicated genes in the healthy and steatotic human liver.

(G) UMAP of murine NAFLD (SD and WD) stromal cells (4,025 cells/nuclei) isolated from Figure S5J and re-clustered with scVI (left) and proportions of each cell type in SD- and WD-fed mice (right).

(H) Top DEGs between cell types.

(I) Mice were fed a western diet (WD) or standard diet (SD) for 36 weeks to induce NAFLD, and Visium analysis was performed. Analysis is pooled from 1 liver slice from the SD condition and 3 liver slices from the WD condition. Zonation of Cc/2 ${ }^{+}$fibroblasts and fibroblasts in SD- and WD-fed mice. See also Tables S3 and S10. 
A

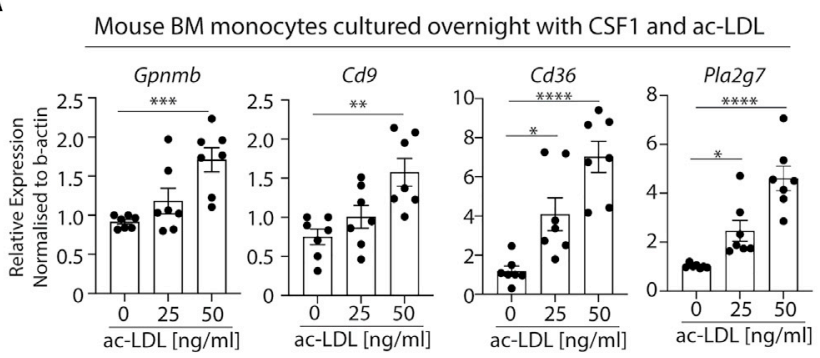

B Human-Mouse Conserved KC Niche Ligand Receptor Pairs

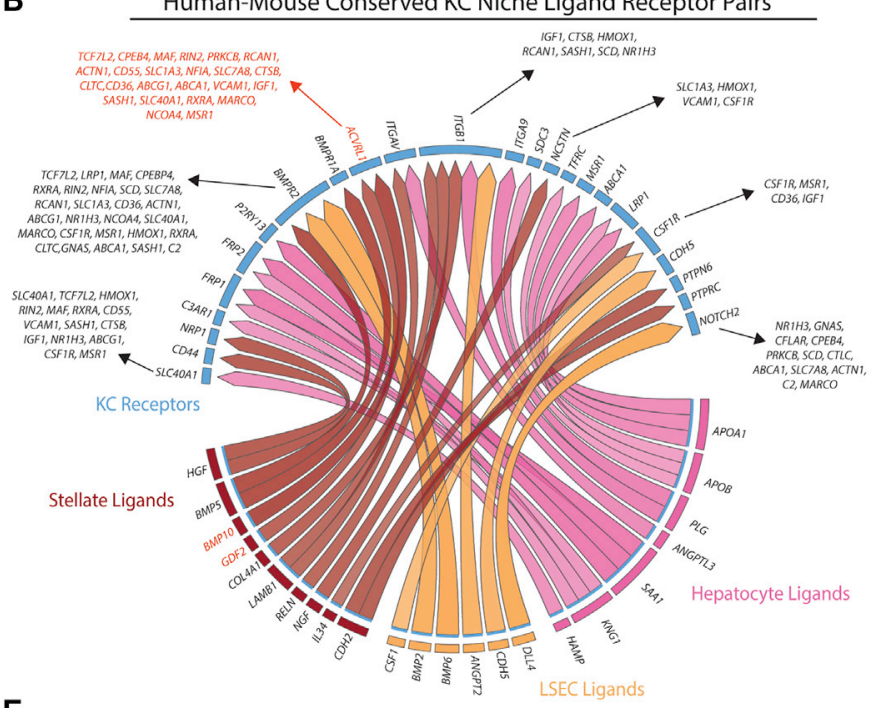

E
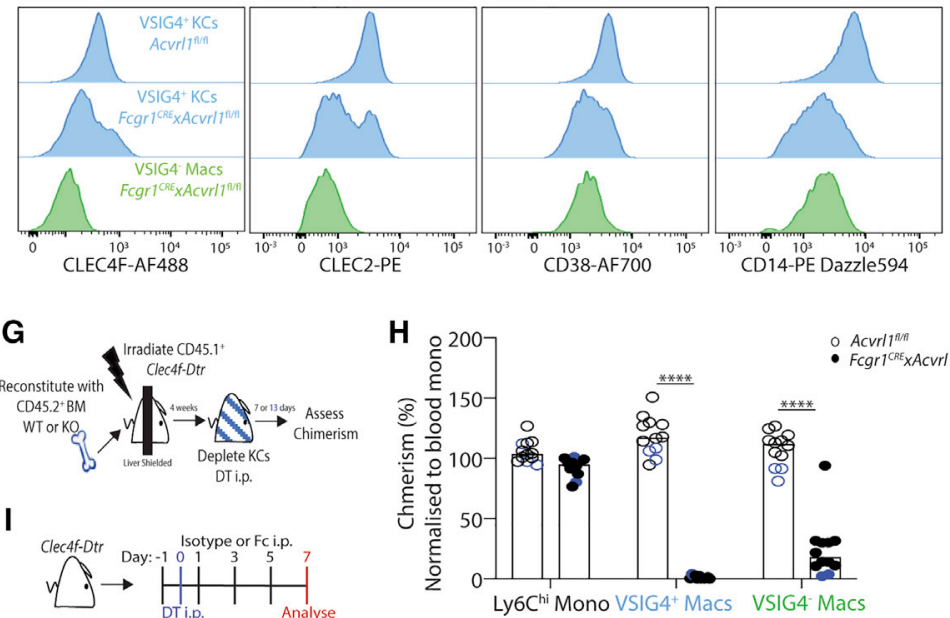

$\mathbf{F}$

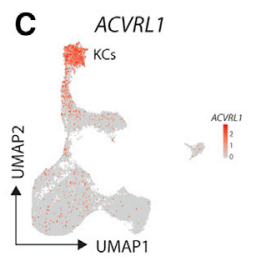

GDF2/BMP10
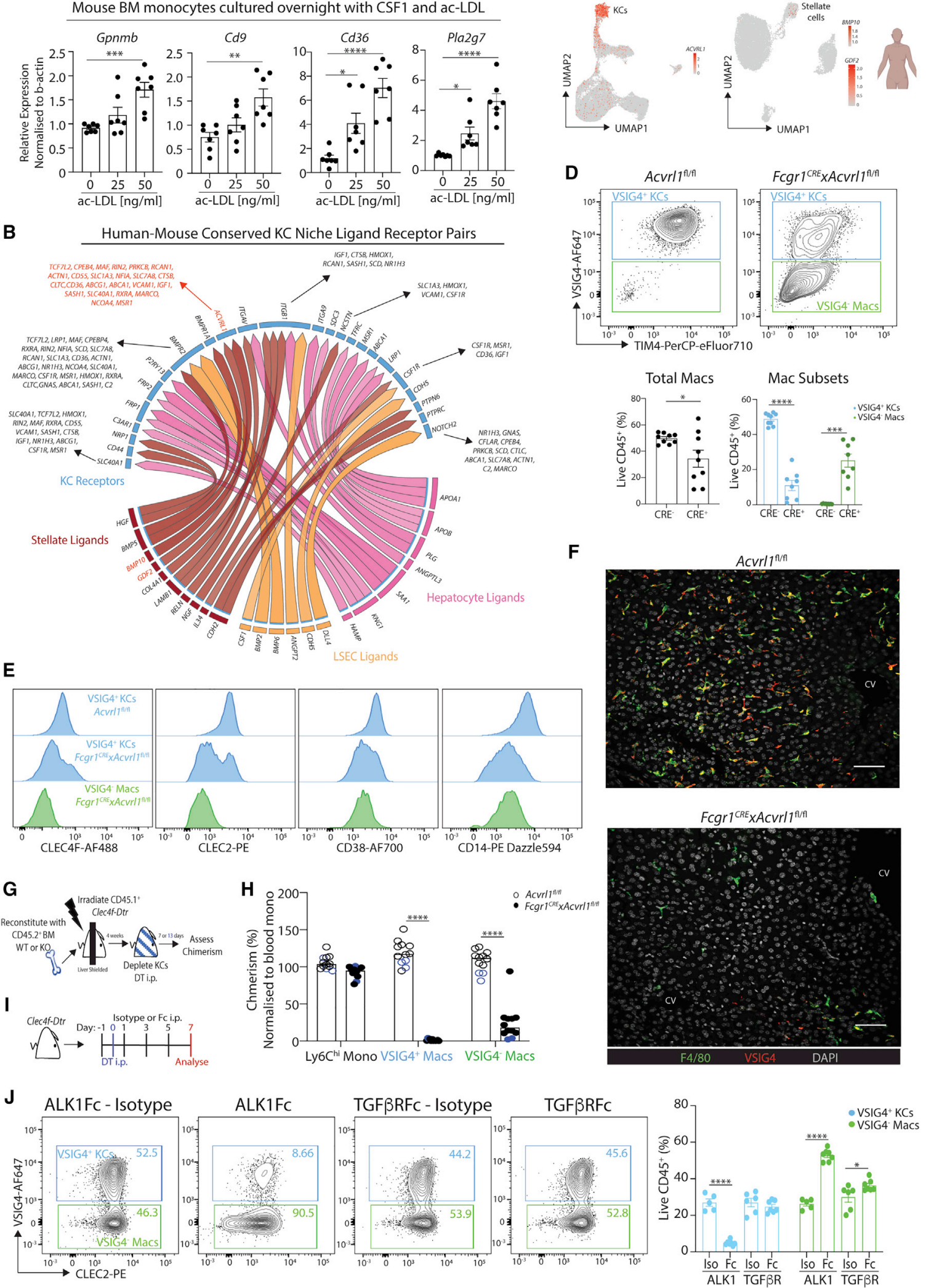

(legend on next page) 
screening broadly, we identify the best surface markers for the isolation and localization of hepatic macs and their respective niche cells. This allows both the validation of the spatial location at the single-cell level, and the efficient screening of transgenic mouse models for the loss of KCs. Characterization of Fcgr1CrexAcvrl $1^{\mathrm{fl} / \mathrm{fl}}$ mice using our defined panel readily demonstrates the cruciality of the ALK1-BMP9/10 axis in KC development emphasizing that mac-stromal-cell cross-talk goes much further than the exchange of growth factors (Guilliams et al., 2020; Zhou et al., 2018). Moving forward, applying these relatively cheap antibody panels to large patient cohorts or multiple transgenic mouse models should enable any perturbations disturbing liver homeostasis to be efficiently identified.

\section{Limitations of the study}

The current study has two main limitations. First, the analysis of the human liver remains restricted by the number of human patients included (19 patients for the sc/snRNA-seq, 4 patients for Visium, and 15 patients by microscopy). While this study provides markers to cheaply and efficiently screen large patient cohorts allowing this analysis to be extended, given the heterogeneity between patients, multiple studies will need to be integrated in a single analysis if we are to be able to interrogate transcriptomic differences in a particular cell subset related to age, sex, ethnicity, or pathological parameters. Second, this study highlights that more research will be needed to fully characterize human SCs. As we could only retrieve these cells through snRNA-seq this means we have not been able to identify good surface markers through CITE-seq. Additionally, for small populations of fibroblasts we did not recover enough nuclei to truly probe their heterogeneity. Better isolation protocols are required to retrieve and enrich these cells from the human liver in order to run a broad panel of CITE-seq markers to identify the different subsets and their corresponding surface markers.

\section{STAR $\star$ METHODS}

Detailed methods are provided in the online version of this paper and include the following:

- KEY RESOURCES TABLE

- RESOURCE AVAILABILITY $\bigcirc$ Lead contact

Materials availability

Data and code availability

- EXPERIMENTAL MODEL AND SUBJECT DETAILS

O In vivo animal studies

O Patient studies

- METHOD DETAILS

Isolation of liver cells

O Isolation of liver nuclei

O Diet induced murine model of NAFLD/NASH

Flow cytometry and cell sorting

Confocal microscopy

O Confocal microscopy combined with RNAScope

Visium

O Visium highly multiplexed protein

- MICS (MACSima ${ }^{\top}$ Imaging Cyclic Staining) technology on the MACSima ${ }^{\top}$ Imaging System by Miltenyi Biotec B.V. \& Co. KG

O Molecular cartography ${ }^{\mathrm{TM}}$

O RNA sequencing, CITE-seq, and qPCR

Preprocessing Visium data

Modelling of Visium data

Differential abundance along zonation

Differential NicheNet

Staining of human liver paraffin sections

O Isolation and culture of BM monocytes with acetylated LDL

O Generation of bone marrow chimeras

O Administration of Fc traps

- QUANTIFICATION AND STATISTICAL ANALYSIS

- ADDITIONAL RESOURCES

\section{SUPPLEMENTAL INFORMATION}

Supplemental information can be found online at https://doi.org/10.1016/j.cell. 2021.12.018.

\section{ACKNOWLEDGMENTS}

We thank all patients and their families for participating in this study. We also thank Janssen for providing the macaque samples; Acceleron Pharma for the kind gift of the ALK1-Fc, TGFBRII-Fc, and isotype controls; the IRC-VIB Flow and Bioimaging core facilities for assistance; VIB Tech Watch and the VIB

Figure 7. ALK1-BMP9/10 axis regulates KC development

(A) Mouse BM monocytes were cultured in the presence of CSF1 and indicated concentrations of human ac-LDL, prior to analyzed for expression of indicated genes by GPCR. Data are pooled from 2 experiments. One-way ANOVA with Bonferroni post-test compared with $0 \mathrm{ng} / \mathrm{mL}$.

(B) NicheNet circos plot highlighting conserved ligand-receptor pairs and induced target genes between $\mathrm{KCs}$ and indicated niche cells in human and mouse.

(C) Feature plots showing expression of ALK1 (Acvrl1) in human myeloid cells (left) and GDF2/BMP10 in CD45- cells (right).

(D) Livers were harvested from Fcgr1-CrexAcvrl1 ${ }^{\mathrm{fl} / \mathrm{fl}}$ mice or Acvrl1 $1^{\mathrm{fl} / \mathrm{fl}}$ or Acvrl1 ${ }^{+/+}$controls and KCs examined (left) and quantified (right) using VSIG4 expression.

(E) Expression of indicated KC markers by mac populations in Fcgr1-CrexAcvrl f ${ }^{f / f f}$ or Acvrf $\left.\right|^{f / f l}$ control mice. Data are pooled from 3 independent experiments with $n=9$ per group. Student's $t$ test.

(F) Expression of indicated markers in livers of Fcgr1-CrexAcvrl $1^{\mathrm{fl} / \mathrm{fl}}$ or $A c v r l 1^{\mathrm{fl} / \mathrm{fl}}$ or $A c v r l 1^{+/+}$control mice by confocal microscopy. Scale bars, $50 \mu \mathrm{m}$. Images are representative of 2 mice per group.

(G) Schematic of chimera experiment setup.

(H) \% chimerism normalized to levels in blood Ly6 $\mathrm{C}^{\text {hi }}$ monocytes in Clec4f-Dtr mice 7 or 13 days after DT administration following partial irradiation and receiving

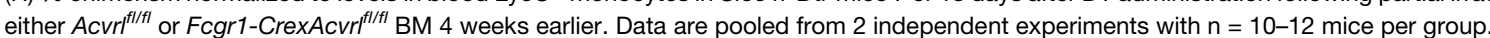

(I) Schematic of Fc trap experiment setup.

(J) Representative FACS plots showing VSIG4 and CLEC2 expression by total macs. Numbers represent \% of total mac population in the indicated gate (left) and $\%$ of VSIG $4^{+}$and VSIG4 ${ }^{-}$macs among total $\mathrm{CD} 45^{+}$cells in the different treatment conditions. One-way ANOVA with Bonferroni post-test. ${ }^{*} \mathrm{p}<0.05,{ }^{* *} \mathrm{p}<0.01$, ${ }^{* * *} \mathrm{p}<0.001,{ }^{* \star *} \mathrm{p}<0.0001$. See also Figure S8. 
single-cell accelerator program for their help benchmarking technologies; and 10X Genomics for their help setting up the Visium highly multiplexed protein analysis. Finally, we thank the VIB-UGent animal house staff. BioRender was used to generate some figures.

Funding: Chan Zuckerberg initiative; liver seed atlas grant (M.G. and C.L.S.), FWO SBO; iPSC LiMics (C.L.S., M.G., and Y.S.), ERC consolidator grant; KupfferCellNiche, 725924 (M.G.), GOA; BOF18-GOA-024 (M.G. and Y.S.), ERC starting grant; MyeFattyLiver, 851908 (C.L.S.), FWO project grant; 3G000519, (C.L.S. and M.G.), FWO PhD fellowship; $1129521 \mathrm{~N}$ (B.H.), 1181318N (R.B.), 11L2122N (F.F.D.P.), MSCA IF fellowships; MACtivate 101027317 (C.Z.), LiverMacRegenCircuit 844301 (F.R.S.).

\section{AUTHOR CONTRIBUTIONS}

Conceptualization \& data curation: C.L.S. and M.G.; formal analysis: C.L.S., M.G., T.T., L.M., B.H., F.R.S., F.F.D.P., A.R., J.B., W.S., and R.B.; funding acquisition: C.L.S. and M.G.; investigation: C.L.S., M.G., B.H., J.B., B.V.D.B., A.B., F.F.D.P., A.R., C.Z., B.V., T.V., L.M., T.T., R.B., A.H., A.V., F.B., Y.V., E.C., G.F., V.W., A.W., S.K., J.N., K.D., P.G., and S.C.; methodology: C.L.S., M.G., B.H., J.B., L.M., T.T., F.F.D.P., R.B., W.S., A.B., A.G., and S.L.; visualization: C.L.S., M.G., J.B., B.H., L.M., T.T., R.B., and W.S.; resources: C.L.S., M.G., W.S., B.D., and Y.S.; supervision: C.L.S., M.G., H.V.V., L.D., B.D., W.S., and Y.S.; writing: C.L.S. and M.G.

\section{DECLARATION OF INTERESTS}

The authors declare no competing interests.

Received: July 2, 2021

Revised: November 12, 2021

Accepted: December 13, 2021

Published: January 11, 2022

\section{REFERENCES}

Aizarani, N., Saviano, A., Sagar, Mailly, L., Durand, S., Herman, J.S., Pessaux, P., Baumert, T.F., and Grün, D. (2019). A human liver cell atlas reveals heterogeneity and epithelial progenitors. Nature 572, 199-204.

Ashburner, M., Ball, C.A., Blake, J.A., Botstein, D., Butler, H., Cherry, J.M., Davis, A.P., Dolinski, K., Dwight, S.S., Eppig, J.T., et al. (2000). Gene ontology: tool for the unification of biology. The Gene Ontology Consortium. Nat. Genet. 25, 25-29.

Berg, S., Kutra, D., Kroeger, T., Straehle, C.N., Kausler, B.X., Haubold, C., Schiegg, M., Ales, J., Beier, T., Rudy, M., et al. (2019). Ilastik: interactive machine learning for (bio)image analysis. Nat. Methods 16, 1226-1232.

Bertrand, J.Y., Chi, N.C., Santoso, B., Teng, S., Stainier, D.Y.R., and Traver, D. (2010). Haematopoietic stem cells derive directly from aortic endothelium during development. Nature 464, 108-111.

Bittmann, I., Bottino, A., Baretton, G.B., Gerbes, A.L., Zachoval, R., Rau, H.G., and Löhrs, U. (2003). The role of graft-resident Kupffer cells and lymphocytes of donor type during the time course after liver transplantation - a clinico-pathological study. Virchows Arch 443, 541-548.

Blériot, C., Barreby, E., Dunsmore, G., Ballaire, R., Chakarov, S., Ficht, X., Simone, G.D., Andreata, F., Fumagalli, V., Guo, W., et al. (2021). A subset of Kupffer cells regulates metabolism through the expression of CD36. Immunity 54, 2101-2116, e6.

Bonnardel, J., T'Jonck, W., Gaublomme, D., Browaeys, R., Scott, C.L., Martens, L., Vanneste, B., Prijck, S.D., Nedospasov, S.A., Kremer, A., et al. (2019). Stellate cells, hepatocytes, and endothelial cells imprint the Kupffer cell identity on monocytes colonizing the liver macrophage niche. Immunity 51, 638-654, e9.

Browaeys, R., Saelens, W., and Saeys, Y. (2019). NicheNet: modeling intercellular communication by linking ligands to target genes. Nat. Methods 17, 159-162.
Chalasani, N., Wilson, L., Kleiner, D.E., Cummings, O.W., Brunt, E.M., and Unalp, A.; NASH Clinical Research Network (2008). Relationship of steatosis grade and zonal location to histological features of steatohepatitis in adult patients with non-alcoholic fatty liver disease. J. Hepatol. 48, 829-834.

Daemen, S., Gainullina, A., Kalugotla, G., He, L., Chan, M.M., Beals, J.W., Liss, K.H., Klein, S., Feldstein, A.E., Finck, B.N., et al. (2021). Dynamic shifts in the composition of resident and recruited macrophages influence tissue remodeling in NASH. Cell Rep 34, 108626.

Desroches-Castan, A., Tillet, E., Bouvard, C., and Bailly, S. (2021). BMP9 and BMP10: two close vascular quiescence partners that stand out. Dev. Dyn. 1-20.

Dobie, R., Wilson-Kanamori, J.R., Henderson, B.E.P., Smith, J.R., Matchett, K.P., Portman, J.R., Wallenborg, K., Picelli, S., Zagorska, A., Pendem, S.V., et al. (2019). Single-cell transcriptomics uncovers zonation of function in the mesenchyme during liver fibrosis. Cell Rep 29, 1832-1847.e8.

Eckert, C., Klein, N., Kornek, M., and Lukacs-Kornek, V. (2015). The complex myeloid network of the liver with diverse functional capacity at steady state and in inflammation. Front. Immunol. 6, 179.

Ellett, F., Pase, L., Hayman, J.W., Andrianopoulos, A., and Lieschke, G.J. (2011). mpeg1 promoter transgenes direct macrophage-lineage expression in zebrafish. Blood 117, e49-e56.

Frankish, A., Diekhans, M., Ferreira, A.-M., Johnson, R., Jungreis, I., Loveland, J., Mudge, J.M., Sisu, C., Wright, J., Armstrong, J., et al. (2019). GENCODE reference annotation for the human and mouse genomes. Nucleic Acids Res 47, D766-D773.

Gans, J.D., and Wolinsky, M. (2008). Improved assay-dependent searching of nucleic acid sequence databases. Nucleic Acids Res 36, e74.

Gayoso, A., Steier, Z., Lopez, R., Regier, J., Nazor, K.L., Streets, A., and Yosef, N. (2021). Joint probabilistic modeling of single-cell multi-omic data with TotalVI. Nat. Methods 18, 272-282.

Gola, A., Dorrington, M.G., Speranza, E., Sala, C., Shih, R.M., Radtke, A.J., Wong, H.S., Baptista, A.P., Hernandez, J.M., Castellani, G., et al. (2021). Commensal-driven immune zonation of the liver promotes host defence. Nature 589, 131-136.

Goumans, M.-J., Valdimarsdottir, G., Itoh, S., Lebrin, F., Larsson, J., Mummery, C., Karlsson, S., and Dijke, P.T. (2003). Activin receptor-like kinase $(\mathrm{ALK}) 1$ is an antagonistic mediator of lateral TGF $\beta / A L K 5$ signaling. Mol. Cell $12,817-828$.

Gu, Z., Gu, L., Eils, R., Schlesner, M., and Brors, B. (2014). circlize implements and enhances circular visualization in R. Bioinformatics 30, 2811-2812.

Guilliams, M., and Scott, C.L. (2017). Does niche competition determine the origin of tissue-resident macrophages? Nat. Rev. Immunol. 17, 415.

Guilliams, M., Thierry, G.R., Bonnardel, J., and Bajénoff, M. (2020). Establishment and maintenance of the macrophage niche. Immunity 52, 434-451.

Haas, J.T., Vonghia, L., Mogilenko, D.A., Verrijken, A., Molendi-Coste, O., Fleury, S., Deprince, A., Nikitin, A., Woitrain, E., Ducrocq-Geoffroy, L., et al. (2019). Transcriptional network analysis implicates altered hepatic immune function in NASH development and resolution. Nat. Metab. 1, 604-614.

Habib, N., Li, Y., Heidenreich, M., Swiech, L., Avraham-Davidi, I., Trombetta, J.J., Hession, C., Zhang, F., and Regev, A. (2016). Div-Seq: single-nucleus RNA-Seq reveals dynamics of rare adult newborn neurons. Science 353, 925-928.

Halpern, K.B., Shenhav, R., Matcovitch-Natan, O., Toth, B., Lemze, D., Golan, M., Massasa, E.E., Baydatch, S., Landen, S., Moor, A.E., et al. (2017). Singlecell spatial reconstruction reveals global division of labour in the mammalian liver. Nature 542, 352-356.

Jaitin, D.A., Adlung, L., Thaiss, C.A., Weiner, A., Li, B., Descamps, H., Lundgren, P., Bleriot, C., Liu, Z., Deczkowska, A., et al. (2019). Lipid-associated macrophages control metabolic homeostasis in a Trem2-dependent manner. Cell 178, 686-698.e14.

Jin, H., Liu, K., Tang, J., Huang, X., Wang, H., Zhang, Q., Zhu, H., Li, Y., Pu, W., Zhao, H., et al. (2021). Genetic fate-mapping reveals surface accumulation but 
not deep organ invasion of pleural and peritoneal cavity macrophages following injury. Nat. Commun. 12, 2863.

Johnson, P., and Ruffell, B. (2009). CD44 and its role in inflammation and inflammatory diseases. Inflamm. Allergy Drug Targets 8, 208-220.

Keren-Shaul, H., Spinrad, A., Weiner, A., Matcovitch-Natan, O., Dvir-Szternfeld, R., Ulland, T.K., David, E., Baruch, K., Lara-Astaiso, D., Toth, B., et al. (2017). A unique microglia type associated with restricting development of Alzheimer's disease. Cell 169, 1276-1290.e17.

Kleiner, D.E., and Makhlouf, H.R. (2016). Histology of nonalcoholic fatty liver disease and nonalcoholic steatohepatitis in adults and children. Clin. Liver Dis. 20, 293-312.

Komesli, S., Vivien, D., and Dutartre, P. (2017). Chimeric extracellular domain of type II transforming growth factor (TGF)- $\beta$ receptor fused to the Fc region of human immunoglobulin as a TGF- $\beta$ antagonist. Eur. J. Biochem. 254, 505-513.

Korsunsky, I., Millard, N., Fan, J., Slowikowski, K., Zhang, F., Wei, K., Baglaenko, Y., Brenner, M., Loh, P.-R., and Raychaudhuri, S. (2019). Fast, sensitive and accurate integration of single-cell data with Harmony. Nat. Methods 16, 1289-1296.

Kuleshov, M.V., Jones, M.R., Rouillard, A.D., Fernandez, N.F., Duan, Q., Wang, Z., Koplev, S., Jenkins, S.L., Jagodnik, K.M., Lachmann, A., et al. (2016). Enrichr: a comprehensive gene set enrichment analysis web server 2016 update. Nucleic Acids Res 44, W90-W97.

Liao, M., Liu, Y., Yuan, J., Wen, Y., Xu, G., Zhao, J., Cheng, L., Li, J., Wang, X., Wang, F., et al. (2020). Single-cell landscape of bronchoalveolar immune cells in patients with COVID-19. Nat. Med. 26, 842-844.

Liao, Y., Wang, J., Jaehnig, E.J., Shi, Z., and Zhang, B. (2019). WebGestalt 2019: gene set analysis toolkit with revamped Uls and APIs. Nucleic Acids Res 47, W199-W205.

Lindeboom, R.G.H., Regev, A., and Teichmann, S.A. (2021). Towards a human cell atlas: taking notes from the past. Trends Genet 37, 625-630.

Lopez, R., Regier, J., Cole, M.B., Jordan, M.I., and Yosef, N. (2018). Deep generative modeling for single-cell transcriptomics. Nat. Methods 15, 1053-1058.

Lun, A.T.L., McCarthy, D.J., and Marioni, J.C. (2016). A step-by-step workflow for low-level analysis of single-cell RNA-seq data with Bioconductor. F1000Res 5, 2122

Marçais, G., and Kingsford, C. (2011). A fast, lock-free approach for efficient parallel counting of occurrences of k-mers. Bioinformatics 27, 764-770.

McCarthy, D.J., Campbell, K.R., Lun, A.T.L., and Wills, Q.F. (2017). Scater: pre-processing, quality control, normalization and visualization of single-cell RNA-seq data in R. Bioinformatics 33, 1179-1186.

Meerschaert, J., and Furie, M.B. (1995). The adhesion molecules used by monocytes for migration across endothelium include CD11a/CD18, CD11b/ CD18, and VLA-4 on monocytes and ICAM-1, VCAM-1, and other ligands on endothelium. J. Immunol. 154, 4099-4112.

Pallett, L.J., Burton, A.R., Amin, O.E., Rodriguez-Tajes, S., Patel, A.A., Zakeri, N., Jeffery-Smith, A., Swadling, L., Schmidt, N.M., Baiges, A., et al. (2020). Longevity and replenishment of human liver-resident memory $T$ cells and mononuclear phagocytes. J. Exp. Med. 217, e20200050.

Park, S.O., Lee, Y.J., Seki, T., Hong, K.-H., Fliess, N., Jiang, Z., Park, A., Wu, X., Kaartinen, V., Roman, B.L., et al. (2008). ALK5- and TGFBR2-independent role of ALK1 in the pathogenesis of hereditary hemorrhagic telangiectasia type 2. Blood 111, 633-642.

Paszke, A., Gross, S., Massa, F., Lerer, A., Bradbury, J., Chanan, G., Killeen, T., Lin, Z., Gimelshein, N., Antiga, L., et al. (2019). PyTorch: an imperative style, high-performance deep learning library. arXiv01703v1, arXiv:1912.

Pont, F., Tosolini, M., and Fournié, J.J. (2019). Single-Cell signature explorer for comprehensive visualization of single cell signatures across scRNA-seq datasets. Nucleic Acids Res 47, e133.
Ramachandran, P., Dobie, R., Wilson-Kanamori, J.R., Dora, E.F., Henderson, B.E.P., Luu, N.T., Portman, J.R., Matchett, K.P., Brice, M., Marwick, J.A., et al. (2019). Resolving the fibrotic niche of human liver cirrhosis at single-cell level. Nature 38, 2-29.

Ranganath, R., Gerrish, S., and Blei, D.M. (2013). Black box variational inference. arXiv, arXiv:1401.0118v1.

Remmerie, A., Martens, L., Thoné, T., Castoldi, A., Seurinck, R., Pavie, B., Roels, J., Vanneste, B., Prijck, S.D., Vanhockerhout, M., et al. (2020). Osteopontin expression identifies a subset of recruited macrophages distinct from Kupffer cells in the fatty liver. Immunity 53, 641-657.e14.

Rizzo, G., Vafadarnejad, E., Arampatzi, P., Silvestre, J.-S., Zernecke, A., Saliba, A.-E., and Cochain, C. (2020). Single-cell transcriptomic profiling maps monocyte/macrophage transitions after myocardial infarction in mice. bioRxiv, bioRxiv, 2020.04.14.040451.

Rodriguez, J.M., Rodriguez-Rivas, J., Di Domenico, T., Vázquez, J., Valencia, A., and Tress, M.L. (2018). APPRIS 2017: principal isoforms for multiple gene sets. Nucleic Acids Res 46, D213-D217.

Sakai, M., Troutman, T.D., Seidman, J.S., Ouyang, Z., Spann, N.J., Abe, Y., Ego, K.M., Bruni, C.M., Deng, Z., Schlachetzki, J.C.M., et al. (2019). Liverderived signals sequentially reprogram myeloid enhancers to initiate and maintain Kupffer cell identity. Immunity 51, 655-670.e8.

Schneider, C.A., Rasband, W.S., and Eliceiri, K.W. (2012). NIH Image to ImageJ: 25 years of image analysis. Nat. Methods 9, 671-675.

Scott, C.L., T'Jonck, W., Martens, L., Todorov, H., Sichien, D., Soen, B., Bonnardel, J., De Prijck, S.D., Vandamme, N., Cannoodt, R., et al. (2018). The transcription factor ZEB2 is required to maintain the tissue-specific identities of macrophages. Immunity 49, 312-325.e5.

Scott, C.L., Zheng, F., De Baetselier, P., Martens, L., Saeys, Y., De Prijck, S., Lippens, S., Abels, C., Schoonooghe, S., Raes, G., et al. (2016). Bone marrowderived monocytes give rise to self-renewing and fully differentiated Kupffer cells. Nat. Commun. 7, 10321.

Sharma, A., Seow, J.J.W., Dutertre, C.-A., Pai, R., Blériot, C., Mishra, A. Wong, R.M.M., Singh, G.S.N., Sudhagar, S., Khalilnezhad, S., et al. (2020) Onco-fetal reprogramming of endothelial cells drives immunosuppressive macrophages in hepatocellular carcinoma. Cell 183, 377-394.e21.

Sierro, F., Evrard, M., Rizzetto, S., Melino, M., Mitchell, A.J., Florido, M., Beattie, L., Walters, S.B., Tay, S.S., Lu, B., et al. (2017). A liver capsular network of monocyte-derived macrophages restricts hepatic dissemination of intraperitoneal bacteria by neutrophil recruitment. Immunity 47, 374-388.e6.

Simone, G.D., Andreata, F., Bleriot, C., Fumagalli, V., Laura, C., Garcia-Manteiga, J.M., Lucia, P.D., Gilotto, S., Ficht, X., Ponti, F.F.D., et al. (2021). Identification of a Kupffer cell subset capable of reverting the $T$ cell dysfunction induced by hepatocellular priming. Immunity 54, 2089-2100.e8.

Stoeckius, M., Hafemeister, C., Stephenson, W., Houck-Loomis, B., Chattopadhyay, P.K., Swerdlow, H., Satija, R., and Smibert, P. (2017). Simultaneous epitope and transcriptome measurement in single cells. Nat. Methods 14, 865-868.

Stuart, T., Butler, A., Hoffman, P., Hafemeister, C., Papalexi, E., Mauck, W.M., Hao, Y., Stoeckius, M., Smibert, P., and Satija, R. (2019). Comprehensive integration of single-cell data. Cell 177, 1888-1902.e21.

Wickham, H. (2016). ggplot2, Elegant Graphics for Data Analysis, Second Edition (Springer).

Wolf, F.A., Angerer, P., and Theis, F.J. (2018). SCANPY: large-scale single-cell gene expression data analysis. Genome Biol 19, 15.

Yates, A.D., Achuthan, P., Akanni, W., Allen, J., Allen, J., Alvarez-Jarreta, J., Amode, M.R., Armean, I.M., Azov, A.G., Bennett, R., et al. (2020). Ensembl 2020. Nucleic Acids Res 48, D682-D688.

Zhou, X., Franklin, R.A., Adler, M., Jacox, J.B., Bailis, W., Shyer, J.A., Flavell, R.A., Mayo, A., Alon, U., and Medzhitov, R. (2018). Circuit design features of a stable two-cell system. Cell 172, 744-757.e17. 


\section{STAR $\star$ METHODS}

\section{KEY RESOURCES TABLE}

\begin{tabular}{|c|c|c|}
\hline REAGENT or RESOURCE & SOURCE & IDENTIFIER \\
\hline \multicolumn{3}{|l|}{ Antibodies: Flow cytometry/confocal microscopy } \\
\hline Rat Monoclonal CD102-FITC (3C4) & Biolegend & 105606; RRID: AB_313199 \\
\hline Rat Monoclonal CD103-BV750 (M290) & BD Biosciences & 747478; RRID: AB_2872154 \\
\hline Rat Monoclonal CD11b-BUV395 (M1/70) & BD Biosciences & 565976; RRID: AB_2721166 \\
\hline Rat Monoclonal CD11b-BV605 (M1/70) & BD Biosciences & 563015; RRID: AB_2737951 \\
\hline Rat Monoclonal MHCII-BUV805 (M5/114,15,2) & BD Biosciences & 748844; RRID: AB_2873247 \\
\hline Rat Monoclonal MHCII-FITC (M5/114,15,2) & Thermo Fisher Scientific & 11-5321-85; RRID: AB_465233 \\
\hline Rat Monoclonal CD14-PE Dazzle 594 (Sa14-2) & Biolegend & 123325; RRID: AB_2721697 \\
\hline Rat Monoclonal CD43-BUV737 (S7) & BD Biosciences & 612840; RRID: AB_2870162 \\
\hline Rat Monoclonal Ly6C-eFluor450 (HK1.4) & eBioscience & 48-5932-82; RRID: AB_10805519 \\
\hline Armenian Hamster Monoclonal CD11C-PE-Cy7 (N418) & eBioscience & 25-0114-82; RRID: AB_469590 \\
\hline Armenian Hamster Monoclonal CD11c-Unconjugated (N418) & Biolegend & 117302; RRID: AB_313771 \\
\hline Rat Monoclonal CD86-BV605 (GL-1) & Biolegend & 105037; RRID: AB_11204429 \\
\hline Rat Monoclonal CD54-PE (YN1/1.7.4) & Biolegend & 116107; RRID: AB_313698 \\
\hline Rat Monoclonal CD54-AF488 (YN1/1,7,4) & Biolegend & 116111; RRID: AB_493494 \\
\hline Rat Monoclonal CD172a-BB630P (P84) & BD Customs & 624294 \\
\hline Mouse Monoclonal XCR1 - BV650 (ZET) & BioLegend & 148220; RRID: AB_2566410 \\
\hline Rat Monoclonal Tim4-PerCP-Cy5.5 (RMT4-54) & eBioscience & 46-5866-82; RRID: AB_2573781 \\
\hline Rat Monoclonal VSIG4-PECy7 (NLA14) & eBioscience & 25-5752-82; RRID: AB_2637431 \\
\hline Rat Monoclonal CD19-PECy5 (1D3) & eBioscience & 15-0193-82; RRID: AB_657672 \\
\hline Rat Monoclonal CD19-Unconjugated (1D3) & BD Biosciences & 553783; RRID: AB_395047 \\
\hline Rat Monoclonal CD26-FITC (H194-112) & BD Biosciences & 559652; RRID: AB_398295 \\
\hline Rat Monoclonal CD38-AF700 (90) & eBioscience & 56-0381-82; RRID: AB_657740 \\
\hline Armenian Hamster Monoclonal CD3e-PECy5 (145-2C11) & TONBO Biosciences & 55-0031; RRID: AB_2621815 \\
\hline Rat Monoclonal CD3-Unconjugated (17A2) & Bioceros & N/A \\
\hline Rat Monoclonal CD45-BV510 (30-F11) & BioLegend & 103138; RRID: AB_2563061 \\
\hline Mouse Monoclonal CD45.1-PE (A20) & BD Biosciences & 553776; RRID: AB_395044 \\
\hline Mouse Monoclonal CD45.1-Unconjugated (A20) & Biolegend & 110702; RRID: AB_313491 \\
\hline Mouse Monoclonal CD45.2-AF700 (104) & eBioscience & 56-0454-82; RRID: AB_657752 \\
\hline Rat Monoclonal CD45R-PE-Cy5 (RA3-6B2) & BD Biosciences & 553091; RRID: AB_394621 \\
\hline Rat Monoclonal Ly6G-BUV563 (1A8) & BD Biosciences & 612921; RRID: AB_2870206 \\
\hline Mouse Monoclonal CD64-BV711 (X54-5/7.1) & BioLegend & 139311; RRID: AB_2563846 \\
\hline Mouse Monoclonal NK1.1-PECy5 (PK136) & BioLegend & 108716; RRID: AB_493590 \\
\hline Rat Monoclonal Ter119-PECy5 (TER-119) & eBioscience & 15-5921-82; RRID: AB_468810 \\
\hline Rat Monoclonal F4/80-BV785 (BM8) & BioLegend & 123141; RRID: AB_2563667 \\
\hline Rat Monoclonal F4/80-Unconjugated (Cl:A3-1) & Bio-Rad & MCA497R; RRID: AB_323279 \\
\hline Rat Monoclonal F4/80-AF594 (BM8) & Biolegend & 123140; RRID: AB_2563241 \\
\hline Rat Monoclonal CLEC2-PE (17D9) & Biolegend & 146104; RRID: AB_2562382 \\
\hline Rat Monoclonal FOLRB-PE (10/FR2) & Biolegend & 153304; RRID: AB_2721344 \\
\hline Rat Monoclonal FOLRB-Unconjugated (10/FR2) & Biolegend & 153302; RRID: AB_2687271 \\
\hline Rat Monoclonal SiglecF-BUV395 (E50-2440) & BD Biosciences & 740280; RRID: AB_2740019 \\
\hline Rat Monoclonal CD207-AF647 & Imgenex & DDX0362A647; RRID: AB_1148741 \\
\hline Rat Monoclonal CD206-AF647 & Biolegend & 141712; RRID: AB_10900420 \\
\hline Rat Monoclonal CD326 (EPCAM)-APC (G8.8) & eBioscience & 17-5791-82; RRID: AB_2716944 \\
\hline
\end{tabular}

(Continued on next page) 


\begin{tabular}{|c|c|c|}
\hline \multicolumn{3}{|l|}{ Continued } \\
\hline REAGENT or RESOURCE & SOURCE & IDENTIFIER \\
\hline Goat Polyclonal Clec4F-Unconjugated & $R$ \& D Systems & AF2784; RRID: AB_2081339 \\
\hline Rat Monoclonal Clec4F-Unconjugated (370901) & $R$ \& D Systems & MAB2784; RRID: AB_2081338 \\
\hline Goat Polyclonal VSIG4-Unconjugated & $R \& D$ Systems & AF4646; RRID: AB_2257239 \\
\hline Goat Polyclonal Lyve1-Unconjugated & R\&D Systems & AF2125; RRID: AB_2297188 \\
\hline Donkey Anti-Goat IgG-AF488 & Thermo Fisher Scientific & A-11055; RRID: AB_2534102 \\
\hline Donkey Anti-Goat IgG-AF633 & Thermo Fisher Scientific & A-21082; RRID: AB_2535739 \\
\hline Donkey Anti-Goat IgG-AF647 & Thermo Fisher Scientific & A-21447; RRID: AB_2535864 \\
\hline Donkey Anti-Rat IgG-Cy3 & Jackson ImmunoResearch & 712-166-153; RRID: AB_2340669 \\
\hline Donkey Anti-Mouse IgG-AF555 & Thermo Fisher Scientific & A-31570; RRID: AB_2536180 \\
\hline Donkey Anti-Rabbit IgG-AF647 & Thermo Fisher Scientific & A-31573; RRID: AB_2536183 \\
\hline Donkey Anti-Rabbit IgG-AF680 & Thermo Fisher Scientific & A-32802; RRID: AB_2762836 \\
\hline Goat Anti-Rabbit IgG-AF514 & Thermo Fisher Scientific & A-31558; RRID: AB_2536173 \\
\hline Goat Anti-Hamster IgG-Cy3 & Jackson ImmunoResearch & 127-165-160; RRID: AB_2338989 \\
\hline Goat Anti-Hamster IgG-AF594 & Jackson ImmunoResearch & 127-585-160; RRID: AB_2338999 \\
\hline Goat Anti-Rat IgG-AF568 & Thermo Fisher Scientific & A-11077; RRID: AB_2534121 \\
\hline Goat Anti-Chicken IgY-AF680 & Abcam & Ab175779; \\
\hline Rat Monoclonal CD31-Unconjugated (MEC13.3) & BD Biosciences & 550274; RRID: AB_393571 \\
\hline Rabbit Polyclonal Desmin-Unconjugated & Abcam & ab15200; RRID: AB_301744 \\
\hline Rabbit Polyclonal Glutamine Synthetase - Unconjugated & Abcam & ab73593; RRID: AB-2247588 \\
\hline Goat Anti-Rabbit IgG-AF514 & Thermo Fisher Scientific & A-31558; RRID: AB_2536173 \\
\hline Rabbit Monoclonal GPNMB- unconjugated (EPR18226-147) & Abcam & ab188222; \\
\hline Polyclonal Chicken anti-GFP & Aves Labs & GFP-1010; RRID: AB_2307313 \\
\hline Mouse Monoclonal FOLR2-PE (94b/FOLR2) & Biolegend & 391704; RRID: AB_2721336 \\
\hline Mouse Monoclonal CD45-APCCy7 (2D1) & Biolegend & 368516; RRID: AB_2566376 \\
\hline Mouse Monoclonal CD14-PETexasRed (TuK4) & Thermo Fisher Scientific & MHCD1417; RRID:AB_10373552 \\
\hline Mouse Monoclonal CD1C-Unconjugated (L161) & Biolegend & 331502; RRID:AB_1088995 \\
\hline Mouse Monoclonal CD163-Unconjugated (10D6) & Thermo Fisher Scientific & MA5-11458; RRID: AB_10982556 \\
\hline Mouse Monoclonal EPCAM-eF660 (1B7) & eBioscience & 50-9326-42: RRID: AB_10598658 \\
\hline Rabbit Monoclonal CD68-Unconjugated (EPR20545) & Abcam & Ab213363; RRID: AB_2801637 \\
\hline Rabbit Monoclonal CD34-PE (EP373Y) & Abcam & Ab223930; \\
\hline Anti- Mouse CD38 (REA616) FITC & Miltenyi Biotec & 130-122-955; RRID: AB_2811415 \\
\hline Anti-Mouse CD26 (REA1196) PE & Miltenyi Biotec & 130-122-775; RRID: AB_2801934 \\
\hline Anti-Mouse F4/80 (REA126) FITC & Miltenyi Biotec & 130-117-509; RRID: AB_2727970 \\
\hline Anti-Mouse CD5 (53-7.3) FITC & Miltenyi Biotec & 130-102-574; RRID: AB_2658608 \\
\hline Anti-Mouse CD79B (REA1117) PE & Miltenyi Biotec & 130-119-425; RRID: AB_2751702 \\
\hline Anti-Mouse CD45R/B220 (RA3-6B2) FITC & Miltenyi Biotec & 130-118-323; RRID: AB_2751482 \\
\hline Anti-Mouse CD43 (L11) PE & Miltenyi Biotec & 130-102-594; RRID: AB_2661309 \\
\hline Anti-Mouse CCR2 (REA538) PE & Miltenyi Biotec & 130-117-548; RRID: AB_2727981 \\
\hline Anti-Mouse MHCII (M5/114.15.2) FITC & Miltenyi Biotec & 130-123-666; RRID: AB_2802055 \\
\hline Anti-Mouse CD73 (REA778) PE & Miltenyi Biotec & 130-111-331; RRID: AB_2659153 \\
\hline Anti-Mouse CD146 (REA1064) FITC & Miltenyi Biotec & 130-118-252; RRID: AB_2751472 \\
\hline Anti-Mouse CD90.2 (30-H12) PE & Miltenyi Biotec & 130-120-091; RRID: AB_2751997 \\
\hline Anti-Mouse CD138 ( REA104) PE & Miltenyi Biotec & 130-120-810; RRID: AB_2752204 \\
\hline Anti-Mouse CD29 (REA1074) PE & Miltenyi Biotec & 130-119-165; RRID: AB_2751649 \\
\hline Anti-Mouse CD105 (REA1058) FITC & Miltenyi Biotec & 130-118-173; RRID: AB_2733613 \\
\hline Anti-Human CD36 (REA760) PE & Miltenyi Biotec & 130-110-877; RRID: AB_2657728 \\
\hline Anti-Human CD206 (REAL518) APC & Miltenyi Biotec & 130-122-168; RRID: AB_2857557 \\
\hline Anti-Human Collagen IV (REAL567) PE & Miltenyi Biotec & 130-122-866; RRID: AB_2857566 \\
\hline
\end{tabular}




\begin{tabular}{|c|c|c|}
\hline \multicolumn{3}{|l|}{ Continued } \\
\hline REAGENT or RESOURCE & SOURCE & IDENTIFIER \\
\hline Anti-Human CD105 (REA794) PE & Miltenyi Biotec & 130-112-163; RRID: AB_2654424 \\
\hline Anti-Human CD146 (REA773) APC & Miltenyi Biotec & 130-111-323; RRID: AB_2655179 \\
\hline Anti-Human CD90 (REA897) PE & Miltenyi Biotec & 130-114-860; RRID: AB_2726811 \\
\hline Anti-Human DESMIN (REA1134) PE & Miltenyi Biotec & 130-119-490; RRID: AB_2857461 \\
\hline Anti-Human CD74 (REA1103) PE & Miltenyi Biotec & 130-119-203; RRID: AB_2733836 \\
\hline Anti-Human EPCAM (REA764) FITC & Miltenyi Biotec & 130-110-998; RRID: AB_2657493 \\
\hline Anti-Human CD68 (REAL566) PE & Miltenyi Biotec & 130-123-368; RRID: AB_2857592 \\
\hline Anti-Human CD1c (REA694) APC & Miltenyi Biotec & 130-110-537; RRID: AB_2656040 \\
\hline Anti-Human CD3 (REA1151) APC & Miltenyi Biotec & 130-120-269; RRID: AB_2876933 \\
\hline Anti-Human CD5 (REA782) PE & Miltenyi Biotec & 130-110-990; RRID: AB_2658593 \\
\hline Anti-Human CD7 (REA1244) PE & Miltenyi Biotec & 130-124-939; RRID: AB_2819716 \\
\hline Anti-Human CD19 (REAL106) PE & Miltenyi Biotec & 130-122-649; RRID: AB_2784034 \\
\hline Anti-Human CD22 (REA340) APC & Miltenyi Biotec & 130-120-762; RRID: AB_2752186 \\
\hline Anti-Human S100A8 (REA917) PE & Miltenyi Biotec & 130-115-253; RRID: AB_2726963 \\
\hline Anti-Human CD177 (REA258) PE & Miltenyi Biotec & 130-101-525; RRID: AB_2655641 \\
\hline Anti-Human CD169 (REA1176) PE & Miltenyi Biotec & 130-121-115; RRID: AB_2783985 \\
\hline Anti-Human CD163 (REA406) PE & Miltenyi Biotec & 130-121-316; RRID: AB_2857545 \\
\hline \multicolumn{3}{|l|}{ Antibodies: CITE-seq } \\
\hline TotalSeq-A0001 anti-mouse CD4 (RM4-5) & BioLegend & 100569; RRID: AB_2749956 \\
\hline TotalSeq-A0002 anti-mouse CD8a (53-6.7) & BioLegend & 100773; RRID: AB_2734151 \\
\hline TotalSeq-A0003 anti-mouse CD366 (Tim-3) (RMT3-23) & BioLegend & 119729; RRID: AB_2734178 \\
\hline TotalSeq-A0004 anti-mouse CD279 (PD-1) (RMP1-30) & BioLegend & 109123; RRID: AB_2734169 \\
\hline TotalSeq-A0005 anti-human CD80 (2D10) & BioLegend & 305239; RRID: AB_2749958 \\
\hline TotalSeq-A0006 anti-human CD86 (IT2.2) & BioLegend & 305443; RRID: AB_2734273 \\
\hline TotalSeq-A0007 anti-human CD274 (29E.2A3) & BioLegend & 329743; RRID: AB_2749959 \\
\hline TotalSeq-A0008 anti-human CD273 (24F.10C112) & BioLegend & 329619; RRID: AB_2734321 \\
\hline TotalSeq-A0009 anti-human CD275 (2D3) & BioLegend & 309413; RRID: AB_2734278 \\
\hline TotalSeq-A0010 anti-human CD276 (DCN.70) & BioLegend & 331607; RRID: AB_2734327 \\
\hline TotalSeq-A0012 anti-mouse CD117 (c-kit) (2B8) & BioLegend & 105843; RRID: AB_2749960 \\
\hline TotalSeq-A0013 anti-mouse Ly-6C (HK1.4) & BioLegend & 128047; RRID: AB_2749961 \\
\hline TotalSeq-A0014 anti-mouse/human CD11b (M1/70) & BioLegend & 101265; RRID: AB_2734152 \\
\hline TotalSeq-A0015 anti-mouse Ly-6G (1A8) & BioLegend & 127655; RRID: AB_2749962 \\
\hline TotalSeq-A0016 anti-human Galectin9 (9M1-3) & BioLegend & Barcode: ACTCACTGGAGTCTC \\
\hline TotalSeq-A0020 anti-human CD270 (122) & BioLegend & 318813; RRID: AB_2734293 \\
\hline TotalSeq-A0021 anti-human CD252 (11C3.1) & BioLegend & Barcode: TITAGTGATCCGACT \\
\hline TotalSeq-A0022 anti-human CD137L (5F4) & BioLegend & 311509; RRID: AB_2734284 \\
\hline TotalSeq-A0023 anti-human CD155 (SKII.4) & BioLegend & 337623; RRID: AB_2749963 \\
\hline TotalSeq-A0024 anti-human CD112 (TX31) & BioLegend & 337417; RRID: AB_2749964 \\
\hline TotalSeq-A0026 anti-human CD47 (CC2C6) & BioLegend & 323129; RRID: AB_2734305 \\
\hline TotalSeq-A0027 anti-human CD70 (113-16) & BioLegend & 355117; RRID: AB_2749965 \\
\hline TotalSeq-A0028 anti-human CD30 (BY88) & BioLegend & 333913; RRID: AB_2749966 \\
\hline TotalSeq-A0029 anti-human CD48 (BJ40) & BioLegend & 336709; RRID: AB_2734342 \\
\hline TotalSeq-A0031 anti-human CD40 (5C3) & BioLegend & 334346; RRID: AB_2749968 \\
\hline TotalSeq-A0032 anti-human CD154 (24-31) & BioLegend & 310843; RRID: AB_2734283 \\
\hline TotalSeq-A0033 anti-human CD52 (Hl186) & BioLegend & 316017; RRID: AB_2734292 \\
\hline TotalSeq-A0034 anti-human CD3 (UCHT1) & BioLegend & 300475; RRID: AB_2734246 \\
\hline TotalSeq-A0047 anti-human CD56 $(5.1 \mathrm{H} 11)$ & BioLegend & 362557; RRID: AB_2749970 \\
\hline TotalSeq-A0050 anti-human CD19 (HIB19) & BioLegend & 302259; RRID: AB_2734256 \\
\hline
\end{tabular}

(Continued on next page) 


\begin{tabular}{|c|c|c|}
\hline \multicolumn{3}{|l|}{ Continued } \\
\hline REAGENT or RESOURCE & SOURCE & IDENTIFIER \\
\hline TotalSeq-A0052 anti-human CD33 (P67.6) & BioLegend & 366629; RRID: AB_2734409 \\
\hline TotalSeq-A0053 anti-human CD11c (S-HCL-3) & BioLegend & 371519; RRID: AB_2749971 \\
\hline TotalSeq-A0054 anti-human CD34 (581) & BioLegend & 343537; RRID: AB_2749972 \\
\hline TotalSeq-A0056 anti-human CD269 (19F2) & BioLegend & 357521; RRID: AB_2749974 \\
\hline TotalSeq-A0057 anti-human B2M (2M2) & BioLegend & 316321; RRID: AB_2749975 \\
\hline TotalSeq-A0058 anti-human HLA-ABC (W6/32) & BioLegend & 311445; RRID: AB_2749976 \\
\hline TotalSeq-A0060 anti-human CD90 $(5 E+10)$ & BioLegend & 328135; RRID: AB_2734312 \\
\hline TotalSeq-A0061 anti-human CD117 (104D2) & BioLegend & 313241; RRID: AB_2734287 \\
\hline TotalSeq-A0062 anti-human CD10 (HI10a) & BioLegend & 312231; RRID: AB_2832627 \\
\hline TotalSeq-A0063 anti-human CD45RA (HI100) & BioLegend & 304157; RRID: AB_2734267 \\
\hline TotalSeq-A0064 anti-human CD123 (6H6) & BioLegend & 306037; RRID: AB_2749977 \\
\hline TotalSeq-A0066 anti-human CD7 (CD7-6B7) & BioLegend & 343123; RRID: AB_2734345 \\
\hline TotalSeq-A0068 anti-human CD105 (43A3) & BioLegend & 323221; RRID: AB_2750350 \\
\hline TotalSeq-A0069 anti-human CD201 (RCR-401) & BioLegend & 351907; RRID: AB_2749978 \\
\hline TotalSeq-A0070 anti-human/mouse CD49f (GoH3) & BioLegend & 313633; RRID; AB_2734291 \\
\hline TotalSeq-A0073 anti-mouse/human CD44 (IM7) & BioLegend & 103045; RRID: AB_2734154 \\
\hline TotalSeq-A0074 anti-mouse CD54 (YN1/1.7.4) & BioLegend & 116127; RRID: AB_2734177 \\
\hline TotalSeq-A0074 anti-mouse CD90.2 (30-H12) & Biolegend & 105345; RRID: AB_2734166 \\
\hline TotalSeq-A0076 anti-mouse/human CD15 (SSEA-1) (MC-480) & BioLegend & 125615; RRID: AB_2800603 \\
\hline TotalSeq-A0077 anti-mouse CD73 (TY/11.8) & BioLegend & 127227; RRID: AB_2749980 \\
\hline TotalSeq-A0078 anti-mouse CD49d (R1-2) & BioLegend & 103623; RRID: AB_2734159 \\
\hline TotalSeq-A0079 anti-mouse CD200 (OX2) (OX-90) & BioLegend & 123811; RRID: AB_2734191 \\
\hline TotalSeq-A0080 anti-human CD8a (RPA-T8) & BioLegend & 301067; RRID: AB_2734248 \\
\hline TotalSeq-A0081 anti-human CD14 (M5E2) & BioLegend & 301855: RRID: AB_2734254 \\
\hline TotalSeq-A0083 anti-human CD16 (3G8) & BioLegend & 302061; RRID: AB_2734255 \\
\hline TotalSeq-A0085 anti-human CD25 (BC96) & BioLegend & 302643; RRID: AB_2734258 \\
\hline TotalSeq-A0087 anti-human CD45RO (UCHL1) & BioLegend & 304255; RRID: AB_2734268 \\
\hline TotalSeq-A0088 anti-human CD279 (EH12.2H7) & BioLegend & 329955; RRID: AB_2734322 \\
\hline TotalSeq-A0089 anti-human TIGIT (A15153G) & BioLegend & 372725; RRID: AB_2734426 \\
\hline TotalSeq-A0090 Mouse IgG1, $\kappa$ isotype Ctrl (MOPC-21) & BioLegend & 400199; RRID: AB_2868412 \\
\hline TotalSeq-A0091 Mouse IgG2a, $\kappa$ isotype Ctrl (MOPC-173) & BioLegend & 400285 \\
\hline TotalSeq-A0092 Mouse IgG2b, $\kappa$ isotype Ctrl (MPC-11) & BioLegend & 400373; \\
\hline TotalSeq-A0093 anti-mouse CD19 (B4) & BioLegend & 115559; RRID: AB_2749981 \\
\hline TotalSeq-A0094 anti-mouse CD3e (145-2C11) & BioLegend & Barcode: TAATGCCAGTTGTGC \\
\hline TotalSeq-A0095 Rat IgG2b, к Isotype Ctrl (RTK4530) & BioLegend & 400673; \\
\hline TotalSeq-A0097 anti-mouse CD25 (PC61) & BioLegend & 102055; RRID: AB_2749982 \\
\hline TotalSeq-A0098 anti-mouse CD135 (A2F10) & BioLegend & 135316; RRID: AB_2749983 \\
\hline TotalSeq-A0100 anti-human CD20 (2H7) & BioLegend & 302359; RRID: AB_2749984 \\
\hline TotalSeq-A0101 anti-human CD335 (900) & BioLegend & 331943; RRID: AB_2800875 \\
\hline TotalSeq-A0102 anti-human CD294 (BM16) & BioLegend & 350127; RRID: AB_2734360 \\
\hline TotalSeq-A0103 anti-mouse/human CD45R/B220 & BioLegend & 103263; RRID: AB_2734158 \\
\hline TotalSeq-A0104 anti-mouse CD102 (3C4 (MIC2/4)) & BioLegend & 105613; RRID: AB_2734167 \\
\hline TotalSeq-A0105 anti-mouse CD115 (CSF-1R) (AFS98) & BioLegend & 135533; RRID: AB_2734198 \\
\hline TotalSeq-A0106 anti-mouse CD11c (N418) & BioLegend & 117355; RRID: AB_2750352 \\
\hline TotalSeq-A0107 anti-mouse CD21/CD35 (CR2/CR1) (7E9) & BioLegend & 123427; RRID: AB_2750540 \\
\hline TotalSeq-A0108 anti-mouse CD23 (B3B4) & BioLegend & 101635; RRID: AB_2750358 \\
\hline TotalSeq-A0109 anti-mouse CD16/32 (93) & BioLegend & 101343; RRID: AB_2750532 \\
\hline TotalSeq-A0110 anti-mouse CD43 (S11) & BioLegend & 143211; RRID: AB_2750541 \\
\hline TotalSeq-A0111 anti-mouse CD5 (53-7.3) & BioLegend & 100637; RRID: AB_2749985 \\
\hline
\end{tabular}




\begin{tabular}{|c|c|c|}
\hline \multicolumn{3}{|l|}{ Continued } \\
\hline REAGENT or RESOURCE & SOURCE & IDENTIFIER \\
\hline TotalSeq-A0112 anti-mouse CD62L (MEL-14) & BioLegend & 104451; RRID: AB_2750364 \\
\hline $\begin{array}{l}\text { TotalSeq-A0113 anti-mouse CD93 (AA4.1, early } \\
\text { B lineage) (AA4.1) }\end{array}$ & BioLegend & 136513; RRID: AB_2750375 \\
\hline TotalSeq-A0114 anti-mouse F4/80 (BM8) & BioLegend & 123153; RRID: AB_2749986 \\
\hline TotalSeq-A0115 anti-mouse FceRl $\alpha$ (MAR-1) & BioLegend & 134333; RRID: AB_2749987 \\
\hline TotalSeq-A0117 anti-mouse I-A/I-E (M5/114.15.2) & BioLegend & 107653; RRID: AB_2750505 \\
\hline TotalSeq-A0118 anti-mouse NK-1.1 (PK136) & BioLegend & 108755; RRID: AB_2750536 \\
\hline TotalSeq-A0119 anti-mouse Siglec H (551) & BioLegend & 129615; RRID: AB_2750537 \\
\hline TotalSeq-A0120 anti-mouse TCRb (H57-597) & Biolegend & 109247; RRID: AB_2750538 \\
\hline TotalSeq-A0121 anti-mouse TCRgd (GL3) & Biolegend & 118137; RRID: AB_2749988 \\
\hline TotalSeq-A0122 anti-mouse Ter-119 (Ter-119) & Biolegend & 116247; RRID: AB_2749989 \\
\hline TotalSeq-A0123 anti-human CD326 (9C4) & BioLegend & 324241; RRID: AB_2750362 \\
\hline TotalSeq-A0124 anti-human CD31 (WM59) & BioLegend & 303137: RRID: AB_2750360 \\
\hline TotalSeq-A0126 anti-human CD133 (clone 7) & BioLegend & 372815; RRID: AB_2750353 \\
\hline TotalSeq-A0127 anti-human Podoplanin (NC-08) & BioLegend & 337019; RRID; AB_2749990 \\
\hline TotalSeq-A0128 anti-human CD140a (16A1) & BioLegend & 323509; RRID: AB_2750354 \\
\hline TotalSeq-A0129 anti-human CD140b (18A2) & BioLegend & 323609; RRID: AB_2783192 \\
\hline TotalSeq-A0130 anti-mouse Ly-6A/E (Sca-1) (D7) & BioLegend & 108147; RRID: AB_2750535 \\
\hline TotalSeq-A0131 anti-human Cadherin (16G5) & BioLegend & 368715; RRID: AB_2749991 \\
\hline TotalSeq-A0132 anti-human EGFR (AY13) & BioLegend & 352923; RRID: AB_2734373 \\
\hline TotalSeq-A0134 anti-human CD146 (P1H12) & BioLegend & 361017: RRID: AB_2750355 \\
\hline TotalSeq-A0136 anti-human IgM (MHM-88) & BioLegend & 314541; RRID: AB_2749992 \\
\hline TotalSeq-A0138 anti-human CD5 (UCHT2) & BioLegend & 300635; RRID: AB_2750346 \\
\hline TotalSeq-A0139 anti-human TCR (B1) & BioLegend & 331229; RRID: AB_2734325 \\
\hline TotalSeq-A0140 anti-human CD183 (G025H7) & BioLegend & 353745; RRID: AB_2749993 \\
\hline TotalSeq-A0141 anti-human CD195 (J418F1) & BioLegend & 359135; RRID: AB_2749994 \\
\hline TotalSeq-A0142 anti-human CD32 (FUN-2) & BioLegend & 303223; RRID: AB_2749995 \\
\hline TotalSeq-A0143 anti-human CD196 (G034E3) & BioLegend & 353437; RRID: AB_2750534 \\
\hline TotalSeq-A0144 anti-human CD185 (J252D4) & BioLegend & 356937; RRID: AB_2750356 \\
\hline TotalSeq-A0145 anti-human CD103 (Ber-ACT8) & BioLegend & 350231; RRID: AB_2749996 \\
\hline TotalSeq-A0146 anti-human CD69 (FN50) & BioLegend & 310947; RRID: AB_2749997 \\
\hline TotalSeq-A0147 anti-human CD62L (DREG-56) & BioLegend & 304847; RRID: AB_2750365 \\
\hline TotalSeq-A0148 anti-human CD197 (G043H7) & BioLegend & 353247; RRID: AB_2750357 \\
\hline TotalSeq-A0149 anti-human CD161 (HP-3G10) & BioLegend & 339945; RRID: AB_2749998 \\
\hline TotalSeq-A0151 anti-human CD152 (BNI3) & BioLegend & 369619; RRID: AB_2734423 \\
\hline TotalSeq-A0152 anti-human CD223 (11C3C65) & BioLegend & 369333; RRID: AB_2749999 \\
\hline TotalSeq-A0153 anti-human KLRG1 (SA231A2) & BioLegend & 367721; RRID: AB_2750373 \\
\hline TotalSeq-A0155 anti-human CD107a (H4A3) & BioLegend & 328647; RRID: AB_2750351 \\
\hline TotalSeq-A0156 anti-human CD95 (DX2) & BioLegend & 305649; RRID: AB_2750368 \\
\hline TotalSeq-A0158 anti-human CD134 (Ber-ACT35) & BioLegend & 350033; RRID: AB_2783245 \\
\hline TotalSeq-A0159 anti-human HLA-DR (L243) & BioLegend & 307659; RRID: AB_2750001 \\
\hline TotalSeq-A0160 anti-human CD1c (L161) & BioLegend & 331539; RRID: AB_2734326 \\
\hline TotalSeq-A0162 anti-human CD64 (10.1) & BioLegend & 305037; RRID: AB_2750366 \\
\hline TotalSeq-A0163 anti-human CD141 (M80) & BioLegend & 344121; RRID: AB_2783229 \\
\hline TotalSeq-A0164 anti-human CD1d (51.1) & BioLegend & 350317; RRID: AB_2750370 \\
\hline TotalSeq-A0165 anti-human CD314 (1D11) & BioLegend & 320835; RRID: AB_2734298 \\
\hline TotalSeq-A0166 anti-human CD66b (6/40c) & BioLegend & 392905; RRID: AB_2750372 \\
\hline TotalSeq-A0167 anti-human CD35 (E11) & BioLegend & 333407; RRID: AB_2783217 \\
\hline TotalSeq-A0168 anti-human CD57 (QA17A04) & BioLegend & 393319; RRID: AB_2810588 \\
\hline
\end{tabular}

(Continued on next page) 


\begin{tabular}{|c|c|c|}
\hline \multicolumn{3}{|l|}{ Continued } \\
\hline REAGENT or RESOURCE & SOURCE & IDENTIFIER \\
\hline TotalSeq-A0169 anti-human CD366 (F38-2E2) & BioLegend & 345047; RRID: AB_2800924 \\
\hline TotalSeq-A0170 anti-human CD272 (MIH26) & BioLegend & 344525; RRID: AB_2750002 \\
\hline $\begin{array}{l}\text { TotalSeq-A0171 anti-human/mouse/rat CD278 (ICOS) } \\
\text { (C398.4A) }\end{array}$ & BioLegend & 313555; RRID: AB_2800824 \\
\hline TotalSeq-A0173 anti-mouse CD206 (MMR) (C068C2) & BioLegend & Barcode: TCAACTCGGTGTTGC \\
\hline TotalSeq-A0174 anti-human CD58 (TS2/9) & BioLegend & 330919; RRID: AB_2750003 \\
\hline TotalSeq-A0175 anti-human CD96 (NK92.39) & BioLegend & 338419; RRID: AB_2750004 \\
\hline TotalSeq-A0176 anti-human CD39 (A1) & BioLegend & 328233; RRID: AB_2750005 \\
\hline TotalSeq-A0177 anti-human CD178 (NOK-1) & BioLegend & 306413; RRID: AB_2750500 \\
\hline TotalSeq-A0180 anti-human CD24 (ML5) & BioLegend & 311137: RRID: AB_2750374 \\
\hline TotalSeq-A0181 anti-human CD21 (Bu32) & BioLegend & 354915; RRID: AB_2750006 \\
\hline TotalSeq-A0182 anti-mouse CD3 (17A2) & Biolegend & 100251; RRID: AB_2750533 \\
\hline TotalSeq-A0184 anti-mouse CD335 (NKp46) (29A1.4) & BioLegend & 137633; RRID: AB_2734199 \\
\hline TotalSeq-A0185 anti-human CD11a (TS2/4) & BioLegend & 350615; RRID: AB_2734365 \\
\hline TotalSeq-A0186 anti-human IgA (HP6123) & BioLegend & Barcode: AAGATGTCCGAGCAA \\
\hline TotalSeq-A0187 anti-human CD79b (CB3-1) & BioLegend & 341415; RRID: AB_2750347 \\
\hline TotalSeq-A0188 anti-human CD66a_c_e (ASL-32) & BioLegend & 342319; RRID: AB_2783223 \\
\hline TotalSeq-A0189 anti-human CD244 (C1.7) & BioLegend & 329527; RRID: AB_2750007 \\
\hline TotalSeq-A0190 anti-mouse CD274 (B7-H1, PD-L1) (MIH6) & BioLegend & 153604; RRID: AB_2783125 \\
\hline TotalSeq-A0191 anti-mouse/rat/human CD27 (LG.3A10) & BioLegend & 124235; RRID: AB_2750344 \\
\hline TotalSeq-A0192 anti-mouse CD20 (SA275A11) & BioLegend & 150423; RRID: AB_2734214 \\
\hline TotalSeq-A0193 anti-mouse CD357 (GITR) (DTA-1) & BioLegend & 126319; RRID: AB_2734195 \\
\hline TotalSeq-A0194 anti-mouse CD137 (17B5) & BioLegend & 106111; RRID: AB_2783048 \\
\hline TotalSeq-A0195 anti-mouse CD134 (OX-40) (OX-86) & BioLegend & 119426; RRID: AB_2750376 \\
\hline TotalSeq-A0196 anti-human CD235ab (HIR2) & BioLegend & 306623; RRID: AB_2750008 \\
\hline TotalSeq-A0197 anti-mouse CD69 (H1.2F3) & BioLegend & 104546; RRID: AB_2750539 \\
\hline TotalSeq-A0198 anti-mouse CD127 (IL-7R $\alpha$ ) (A7R34) & BioLegend & 135045; RRID: AB_2750009 \\
\hline TotalSeq-A0200 anti-mouse CD86 (GL-1) & BioLegend & 105047; RRID: AB_2750348 \\
\hline TotalSeq-A0201 anti-mouse CD103 (2E7) & BioLegend & 121437; RRID: AB_2750349 \\
\hline TotalSeq-A0202 anti-mouse CD64 (FcrRI) (X54-5/7.1) & BioLegend & 139325; RRID: AB_2750367 \\
\hline TotalSeq-A0203 anti-mouse CD150 (SLAM) (TC15-12F12.2) & BioLegend & 115945; RRID: AB_2783055 \\
\hline TotalSeq-A0204 anti-mouse CD28 (37.51) & BioLegend & Barcode: ATTAAGAGCGTGTTG \\
\hline TotalSeq-A0205 anti-human CD206 (15-2) & BioLegend & 321143; RRID: AB_2750010 \\
\hline TotalSeq-A0206 anti-human CD169 (7-239) & BioLegend & 346011; RRID: AB_2750011 \\
\hline TotalSeq-A0207 anti-human CD370 (8F9) & BioLegend & 353807; RRID: AB_2814293 \\
\hline TotalSeq-A0208 anti-human XCR1 (S15046E) & BioLegend & 372613; RRID: AB_2783286 \\
\hline TotalSeq-A0209 anti-mouse TCR (2.11) & BioLegend & 141113; RRID: AB_2800654 \\
\hline TotalSeq-A0210 anti-mouse TCR (536) & BioLegend & 137507; RRID: AB_2810404 \\
\hline TotalSeq-A0211 anti-mouse TCR (UC3-10A6) & BioLegend & 137709; RRID: AB_2783100 \\
\hline TotalSeq-A0212 anti-mouse CD24 (M1/69) & BioLegend & 101841; RRID: AB_2750380 \\
\hline TotalSeq-A0213 anti-human Notch (MHN1-519) & BioLegend & 352109; RRID: AB_2783247 \\
\hline TotalSeq-A0214 anti-human/mouse integrin $\beta 7$ (FIB504) & BioLegend & 321227; RRID: AB_2750504 \\
\hline TotalSeq-A0215 anti-human CD268 (11C1) & BioLegend & 316925; RRID: AB_2750502 \\
\hline TotalSeq-A0216 anti-human CD42b (HIP1) & BioLegend & 303937; RRID: AB_2783163 \\
\hline TotalSeq-A0217 anti-human CD54 (HA58) & BioLegend & 353123; RRID: AB_2750384 \\
\hline TotalSeq-A0218 anti-human CD62P (AK4) & BioLegend & 304933; RRID: AB_2750386 \\
\hline TotalSeq-A0219 anti-human CD119 (GIR-208) & BioLegend & 308607; RRID: AB_2750385 \\
\hline TotalSeq-A0221 anti-human IRF5 (11F4A09) & BioLegend & Barcode: GGTAGCCCTAGAGTA \\
\hline TotalSeq-A0224 anti-human TCR (IP26) & BioLegend & 306737: RRID: AB_2783167 \\
\hline
\end{tabular}




\begin{tabular}{|c|c|c|}
\hline \multicolumn{3}{|l|}{ Continued } \\
\hline REAGENT or RESOURCE & SOURCE & IDENTIFIER \\
\hline TotalSeq-A0225 anti-mouse CD196 (CCR6) (29-2L17) & BioLegend & 129825; RRID: AB_2783083 \\
\hline TotalSeq-A0226 anti-mouse CD106 (429 (MVCAM.A)) & BioLegend & 105725; RRID: AB_2783044 \\
\hline TotalSeq-A0227 anti-mouse CD122 (IL-2Rb) (5H4) & BioLegend & Barcode: GGTATGCGACACTTA \\
\hline TotalSeq-A0228 anti-mouse CD183 (CXCR3) (CXCR3-173) & BioLegend & Barcode: GTTCACGCCGTAGACT \\
\hline TotalSeq-A0229 anti-mouse CD62P (P-selectin) (RMP-1) & BioLegend & Barcode: TGTGTGCCGTAGACT \\
\hline TotalSeq-A0230 anti-mouse CD8b (Ly-3) (YTS156.7.7) & BioLegend & 126623; RRID: AB_2800615 \\
\hline TotalSeq-A0232 anti-mouse MAdCAM-1 (MECA-367) & BioLegend & 120713; RRID: AB_2783058 \\
\hline TotalSeq-A0235 anti-mouse TCR (KJ16-133.18) & BioLegend & 118415; RRID: AB_2783056 \\
\hline TotalSeq-A0236 Rat IgG1, к Isotype Ctrl (RTK2071) & BioLegend & 400459 \\
\hline TotalSeq-A0237 Rat IgG1, $\lambda$ Isotype Ctrl (G0114F7) & BioLegend & 401919; \\
\hline TotalSeq-A0238 Rat IgG2a, к Isotype Ctrl (RTK2758) & BioLegend & 400571; \\
\hline TotalSeq-A0240 Purified Rat IgG2c, к Isotype Ctrl (RTK4174) & BioLegend & 400739; \\
\hline TotalSeq-A0241 Armenian Hamster IgG Isotype Ctrl (HTK888) & BioLegend & 400973; \\
\hline TotalSeq-A0242 anti-human CD192 (K036C2) & BioLegend & 357229; RRID: AB_2750501 \\
\hline TotalSeq-A0244 anti-human CD102 (CBR-IC2/2) & BioLegend & 328509; RRID: AB_2750381 \\
\hline TotalSeq-A0245 anti-human CD106 (STA) & BioLegend & 305813; RRID: AB_2800788 \\
\hline TotalSeq-A0247 anti-human CD267 (1A1) & BioLegend & 311913; RRID: AB_2783181 \\
\hline TotalSeq-A0248 anti-human CD62E (HAE-1f) & BioLegend & 336017; RRID: AB_2783218 \\
\hline TotalSeq-A0249 anti-mouse/human IRF4 (IRF4.3E4) & BioLegend & Barcode: GGATTTGTATCTCCC \\
\hline $\begin{array}{l}\text { TotalSeq-A0250 anti-mouse/human KLRG1 (MAFA) } \\
\text { (2F1/KLRG1) }\end{array}$ & BioLegend & 138431; RRID: AB_2800648 \\
\hline TotalSeq-A0351 anti-human CD135 (BV10A4H2) & BioLegend & 313317; RRID: AB_2783188 \\
\hline TotalSeq-A0352 anti-human FCeR1a (AER-37) & BioLegend & 334641; RRID: AB_2750503 \\
\hline TotalSeq-A0353 anti-human CD41 (HIP8) & BioLegend & 303737; RRID: AB_2783162 \\
\hline TotalSeq-A0354 anti-mouse TCR (MR9-4) & BioLegend & 139517; RRID: AB_2810408 \\
\hline TotalSeq-A0355 anti-human CD137 (4B4-1) & BioLegend & 309835; RRID: AB_2783173 \\
\hline TotalSeq-A0356 anti-human CD254 (MIH24) & BioLegend & 347509; RRID: AB_2750377 \\
\hline TotalSeq-A0357 anti-human CD43 (CD43-10G7) & BioLegend & 343209; RRID: AB_2800915 \\
\hline TotalSeq-A0358 anti-human CD163 (GHI/61) & BioLegend & 333635; RRID: AB_2750343 \\
\hline TotalSeq-A0359 anti-human CD83 (HB15e) & BioLegend & 305339; RRID: AB_2800784 \\
\hline TotalSeq-A0360 anti-human CD357 (108-17) & BioLegend & 371225; RRID: AB_2801016 \\
\hline TotalSeq-A0361 anti-human CD59 (p282 (H19)) & BioLegend & 304709; RRID: AB_2750371 \\
\hline TotalSeq-A0362 anti-human CD309 (7D4-6) & BioLegend & 359919; RRID: AB_2810571 \\
\hline TotalSeq-A0364 anti-human CD13 (WM15) & BioLegend & 301729; RRID: AB_2783151 \\
\hline TotalSeq-A0366 anti-human CD184 (12G5) & BioLegend & 306531; RRID: AB_2800790 \\
\hline TotalSeq-A0367 anti-human CD2 (TS1/8) & BioLegend & Barcode: TACGATTTGTCAGGG \\
\hline TotalSeq-A0368 anti-human CD226 (11A8) & BioLegend & 338335; RRID: AB_2783220 \\
\hline TotalSeq-A0369 anti-human CD29 (TS2/16) & BioLegend & 303027; RRID: AB_2783160 \\
\hline TotalSeq-A0370 anti-human CD303 (201A) & BioLegend & 354239; RRID: AB_2800952 \\
\hline TotalSeq-A0371 anti-human CD49b (P1E6-C5) & BioLegend & 359311; RRID: AB_2750363 \\
\hline TotalSeq-A0373 anti-human CD81 (5A6) & BioLegend & 349521; RRID: AB_2800930 \\
\hline TotalSeq-A0374 anti-human CD98 (MEM-108) & BioLegend & 315605; RRID: AB_2750369 \\
\hline TotalSeq-A0375 anti-human IgG (M1310G05) & BioLegend & 410725; RRID: AB_2783329 \\
\hline TotalSeq-A0376 anti-mouse CD195 (CCR5) (HM-CCR5) & BioLegend & 107019; RRID: AB_2783049 \\
\hline TotalSeq-A0377 anti-mouse CD197 (CCR7) (4B12) & BioLegend & Barcode: TTATTAACAGCCCAC \\
\hline TotalSeq-A0378 anti-mouse CD223 (LAG-3) (C9B7W) & BioLegend & 125229; RRID: AB_2783078 \\
\hline $\begin{array}{l}\text { TotalSeq-A0379 anti-mouse CD62E (E-selectin) } \\
\text { (RME-1/CD62E) }\end{array}$ & BioLegend & Barcode: CTCCCTTTGTAACAT \\
\hline TotalSeq-A0381 anti-mouse Panendothelial Cell & BioLegend & 120507; RRID: AB_2783057 \\
\hline
\end{tabular}




\begin{tabular}{|c|c|c|}
\hline \multicolumn{3}{|l|}{ Continued } \\
\hline REAGENT or RESOURCE & SOURCE & IDENTIFIER \\
\hline TotalSeq-A0382 anti-human CD177 (MEM-166) & BioLegend & 315811: RRID: AB_2750554 \\
\hline TotalSeq-A0383 anti-human CD55 (JS11) & BioLegend & 311317; RRID: AB_2750378 \\
\hline TotalSeq-A0384 anti-human IgD (IA6-2) & BioLegend & 348243; RRID: AB_2783238 \\
\hline TotalSeq-A0385 anti-human CD18 (TS1/18) & BioLegend & 302121; RRID: AB_2750382 \\
\hline TotalSeq-A0386 anti-human CD28 (CD28.2) & BioLegend & 302955; RRID: AB_2783159 \\
\hline TotalSeq-A0387 anti-human TSLPR (1D3) & BioLegend & 322907; RRID: AB_2800845 \\
\hline TotalSeq-A0388 anti-mouse CD152 (UC10-4B9) & BioLegend & 106325; RRID: AB_2876417 \\
\hline TotalSeq-A0389 anti-human CD38 (HIT2) & BioLegend & 303541; RRID: AB_2783161 \\
\hline TotalSeq-A0390 anti-human CD127 (A019D5) & BioLegend & 351352; RRID: AB_2734366 \\
\hline TotalSeq-A0392 anti-human CD15 (W6D3) & BioLegend & 323046; RRID: AB_2734304 \\
\hline TotalSeq-A0393 anti-human CD22 (S-HCL-1) & BioLegend & 363514; RRID: AB_2734404 \\
\hline TotalSeq-A0394 anti-human CD71 (CY1G4) & BioLegend & 334123; RRID: AB_2800884 \\
\hline TotalSeq-A0395 anti-human B7H4 (MIH43) & BioLegend & 358114; RRID: AB_2734386 \\
\hline TotalSeq-A0396 anti-human CD26 (BA5b) & BioLegend & 302720; RRID: AB_2734261 \\
\hline TotalSeq-A0397 anti-human CD193 (5e7) & BioLegend & 310729; RRID: AB_2783174 \\
\hline TotalSeq-A0398 anti-human CD115 (9-4D2-1E4) & BioLegend & 347325; RRID: AB_2783237 \\
\hline TotalSeq-A0399 anti-human CD204 (7C9C20) & BioLegend & 371909; RRID: AB_2810584 \\
\hline TotalSeq-A0400 anti-human CD144 (BV9) & BioLegend & 348517; RRID: AB_2783239 \\
\hline TotalSeq-A0401 anti-human CD301 (H037G3) & BioLegend & 354707; RRID: AB_2810566 \\
\hline TotalSeq-A0402 anti-human CD1a (HI149) & BioLegend & 300133; RRID: AB_2783146 \\
\hline TotalSeq-A0404 anti-human CD63 (H5C6) & BioLegend & 353035; RRID: AB_2783249 \\
\hline TotalSeq-A0405 anti-human CD284 (HTA125) & BioLegend & 312817; RRID: AB_2783183 \\
\hline TotalSeq-A0406 anti-human CD304 (12C2) & BioLegend & 354525; RRID: AB_2783261 \\
\hline TotalSeq-A0407 anti-human CD36 (5-271) & BioLegend & 336225; RRID: AB_2800892 \\
\hline TotalSeq-A0408 anti-human CD172a (15-414) & BioLegend & 372109; RRID: AB_2783285 \\
\hline TotalSeq-A0409 anti-human CD85g (17G10.2 & BioLegend & 326411; RRID: AB_2750555 \\
\hline TotalSeq-A0411 Polyclonal Clec4F (Custom) & BioLegend & Barcode: TATGCTGTGGCTATG \\
\hline TotalSeq-A0414 Polyclonal VSIG4 (Custom) & BioLegend & Barcode: ACGTATATCACGTTG \\
\hline TotalSeq-A0415 anti-P2RY12 (S16007D) & BioLegend & 848009; RRID: AB_2783419 \\
\hline TotalSeq-A0416 anti-mouse CD300LG (Nepmucin) (ZAQ5) & BioLegend & 147105; RRID: AB_2783116 \\
\hline TotalSeq-A0417 anti-mouse CD163 (S16007D) & BioLegend & 155303; RRID: AB_2814058 \\
\hline TotalSeq-A0418 anti-human CD243 (4E3.16) & BioLegend & 919407: RRID: AB_2810817 \\
\hline TotalSeq-A0419 anti-human CD72 (3F3) & BioLegend & 316205; RRID: AB_2783189 \\
\hline TotalSeq-A0420 anti-human CD158 (HP-MA4) & BioLegend & 339515; RRID: AB_2800901 \\
\hline TotalSeq-A0421 anti-mouse CD49b (HMa2) & BioLegend & 103523; RRID: AB_2819796 \\
\hline TotalSeq-A0422 anti-mouse CD172a (SIRP $\alpha$ ) (P84) & BioLegend & 144033; RRID: AB_2800670 \\
\hline TotalSeq-A0423 anti-human MerTK (5900H11G1E3) & BioLegend & 367617; RRID: AB_2801011 \\
\hline TotalSeq-A0424 anti-mouse CD14 (Sa14-2) & BioLegend & 123333; RRID: AB_2800591 \\
\hline TotalSeq-A0426 anti-mouse CD192 (CCR2) (SA203G11) & BioLegend & 150625; RRID: AB_2783122 \\
\hline TotalSeq-A0427 anti-human FOLR2 (94b/FOLR2) & BioLegend & 391707; RRID: AB_2783289 \\
\hline TotalSeq-A0428 anti-human TIM4 (9F4) & BioLegend & 354009; RRID: AB_2783258 \\
\hline TotalSeq-A0429 anti-mouse CD48 (HM48.1) & BioLegend & 103447; RRID: AB_2800558 \\
\hline TotalSeq-A0430 anti-human CD171 (L1-OV198.5) & BioLegend & 371609; RRID: AB_2801019 \\
\hline TotalSeq-A0432 anti-human CD320 (3F4) & BioLegend & 800319; RRID: AB_2801138 \\
\hline TotalSeq-A0433 anti-human CD325 (8C11) & BioLegend & 350817; RRID: AB_2810555 \\
\hline TotalSeq-A0434 anti-mouse/human ReceptorD4 & BioLegend & Barcode: TCTCTGCACCGCTTT \\
\hline TotalSeq-A0435 anti-mouse/human GABRB3 & BioLegend & Barcode: GGTGTGAGCAGTTCT \\
\hline TotalSeq-A0437 anti-mouse/human CD207 (4C7) & BioLegend & Barcode: CGATTTGTATTCCCT \\
\hline TotalSeq-A0438 anti-mouse/rat KKC2 & BioLegend & Barcode: GAGCTTGTACCGCTT \\
\hline
\end{tabular}




\begin{tabular}{|c|c|c|}
\hline \multicolumn{3}{|l|}{ Continued } \\
\hline REAGENT or RESOURCE & SOURCE & IDENTIFIER \\
\hline TotalSeq-A0439 anti-mouse CD201 (RCR-16) & BioLegend & 141509; RRID: AB_2800655 \\
\hline TotalSeq-A0440 anti-mouse CD169 (Siglec-1) (N1/12) & BioLegend & 142425; RRID: AB_2783106 \\
\hline TotalSeq-A0441 anti-mouse CD71 (3D6.112) & BioLegend & 113824; RRID: AB_2800574 \\
\hline TotalSeq-A0442 anti-mouse Notch (HMN1-12) & BioLegend & 130617; RRID: AB_2783085 \\
\hline TotalSeq-A0443 anti-mouse CD41 (MWReg30) & BioLegend & 133937; RRID: AB_2800635 \\
\hline TotalSeq-A0444 anti-mouse CXCR4 (L276F12) & BioLegend & 146520; RRID: AB_2800682 \\
\hline TotalSeq-A0445 anti-human TMEM119 (A16075D) & BioLegend & 853303; RRID: AB_2801201 \\
\hline TotalSeq-A0446 anti-human CD93 (VIMD2) & BioLegend & 336121; RRID: AB_2750379 \\
\hline TotalSeq-A0448 anti-mouse CD204 (Msr1) (1F8C33) & BioLegend & 154703; RRID: AB_2783126 \\
\hline TotalSeq-A0449 anti-mouse CD326 (Ep-CAM) (G8.8) & BioLegend & 118237; RRID: AB_2800586 \\
\hline TotalSeq-A0450 anti-mouse IgM (RMM-1) & BioLegend & 406535; RRID: AB_2783322 \\
\hline TotalSeq-A0551 anti-mouse CD301a (MGL1) (LOM-8.7) & BioLegend & 145611; RRID: AB_2783114 \\
\hline TotalSeq-A0552 anti-mouse CD304 (Neuropilin-1) (3E12) & BioLegend & 145215; RRID: AB_2750383 \\
\hline TotalSeq-A0554 anti-mouse CD309 (VEGFR2, Flk-1) (89B3A5) & BioLegend & 121921; RRID: AB_2783066 \\
\hline TotalSeq-A0555 anti-mouse CD36 (HM36) & BioLegend & 102621; RRID: AB_2800557 \\
\hline TotalSeq-A0556 anti-mouse CD370 (CLEC9A-DNGR1) (7H11) & BioLegend & Barcode: AACTCAGTTGTGCCG \\
\hline TotalSeq-A0557 anti-mouse CD38 (90) & BioLegend & 102733; RRID: AB_2750556 \\
\hline TotalSeq-A0558 anti-mouse CD55 (DAF) ( RIKO-3) & BioLegend & 131809; RRID: AB_2783086 \\
\hline TotalSeq-A0559 anti-mouse CD63 (NVG-2) & BioLegend & 143915; RRID: AB_2783109 \\
\hline TotalSeq-A0560 anti-mouse CD68 (FA-11) & BioLegend & 137031; RRID: AB_2783099 \\
\hline TotalSeq-A0561 anti-mouse CD79b (lg $\beta)(H M 79-12)$ & BioLegend & 132811; RRID: AB_2783087 \\
\hline TotalSeq-A0562 anti-mouse CD83 (Michel-19) & BioLegend & 121519; RRID: AB_2783061 \\
\hline TotalSeq-A0563 anti-mouse CX3CR1 (SA011F11) & BioLegend & 149041; RRID: AB_2783121 \\
\hline TotalSeq-A0564 anti-mouse Folate Receptor $\beta$ (FR- $\beta$ ) (10/FR2) & BioLegend & 153307; RRID: AB_2800690 \\
\hline TotalSeq-A0565 anti-mouse MERTK (Mer) (2B10C42) & BioLegend & Barcode: AGTAGAGCAACTCGT \\
\hline TotalSeq-A0566 anti-mouse CD301b (MGL2) (URA-1) & BioLegend & 146817; RRID: AB_2783115 \\
\hline TotalSeq-A0567 anti-mouse Tim-4 (RMT4-54) & BioLegend & 130011; RRID: AB_2783084 \\
\hline TotalSeq-A0568 anti-mouse/rat XCR1 (ZET) & BioLegend & 148227; RRID: AB_2783120 \\
\hline TotalSeq-A0569 anti-human CD338 (5D3) & BioLegend & 332021; RRID: AB_2783216 \\
\hline TotalSeq-A0570 anti-mouse/rat CD29 (HM $\beta 1-1)$ & BioLegend & 102233; RRID: AB_2783042 \\
\hline TotalSeq-A0571 anti-mouse IgD (11-26c.2a) & BioLegend & 405745; RRID: AB_2783321 \\
\hline TotalSeq-A0572 anti-human C5L2 (1D(-M12) & BioLegend & 342407; RRID: 2783226 \\
\hline TotalSeq-A0573 anti-mouse CD140a (APA5) & BioLegend & 135917; RRID: AB_2783094 \\
\hline TotalSeq-A0574 anti-human CD235a (HI264) & BioLegend & 349117; RRID: AB_2783242 \\
\hline TotalSeq-A0575 anti-human CD49a (TS2/7) & BioLegend & 328315; RRID: AB_2783195 \\
\hline TotalSeq-A0576 anti-human CD49d (9F10) & BioLegend & 304337; RRID: AB_2783166 \\
\hline TotalSeq-A0577 anti-human CD73 (AD2) & BioLegend & 344029; RRID: AB_2783228 \\
\hline TotalSeq-A0578 anti-human CD79a & BioLegend & Barcode: CTTATCACCCGCTIT \\
\hline TotalSeq-A0579 anti-human CD9 (H19a) & BioLegend & 312119; RRID: AB_2783182 \\
\hline TotalSeq-A0580 anti-human mast cell tryptase & BioLegend & Barcode: ACTGATAGACCCGCT \\
\hline TotalSeq-A0581 anti-human TCR (3C10) & BioLegend & 351733; RRID: AB_2783246 \\
\hline TotalSeq-A0582 anti-human TCR (B6) & BioLegend & 331433; RRID: AB_2800863 \\
\hline TotalSeq-A0583 anti-human TCR (B3) & BioLegend & 331311; RRID: AB_2783207 \\
\hline TotalSeq-A0584 anti-human TCR (6B11) & BioLegend & 342923; RRID: AB_2783227 \\
\hline TotalSeq-A0586 anti-human CD354 (Trem-26) & BioLegend & 314910 \\
\hline TotalSeq-A0588 anti-human CD202b (HIT2) & BioLegend & 334213; RRID: AB_2810511 \\
\hline TotalSeq-A0590 anti-human CD305 (NKTA255) & BioLegend & 342805; RRID: AB_2800911 \\
\hline TotalSeq-A0591 anti-human LOX1 (15C4) & BioLegend & 358611; RRID: AB_2800987 \\
\hline TotalSeq-A0592 anti-human CD158b (DX27) & BioLegend & 312615; RRID: AB_2800818 \\
\hline
\end{tabular}




\begin{tabular}{|c|c|c|}
\hline \multicolumn{3}{|l|}{ Continued } \\
\hline REAGENT or RESOURCE & SOURCE & IDENTIFIER \\
\hline TotalSeq-A0593 anti-human CD203c (NP4D6) & BioLegend & 324627; RRID: AB_2800849 \\
\hline TotalSeq-A0595 anti-mouse CD11a (M17/4) & BioLegend & 101125; RRID: AB_2783036 \\
\hline TotalSeq-A0596 anti-mouse ESAM (1G8/ESAM) & BioLegend & 136209; RRID: AB_2800642 \\
\hline TotalSeq-A0597 anti-human CD209 (9E9A8) & BioLegend & 330119; RRID: AB_2783206 \\
\hline TotalSeq-A0599 anti-human CD158e1 (DX9) & BioLegend & 312723; RRID: AB_2800819 \\
\hline TotalSeq-A0600 anti-human CD158f (UP-R1) & BioLegend & 341307; RRID: AB_2800904 \\
\hline TotalSeq-A0801 anti-human CD337 (P30-15) & BioLegend & 325221; RRID: AB_2800852 \\
\hline TotalSeq-A0803 anti-human CD253 (RIK-2) & BioLegend & 308211; RRID: AB_2800803 \\
\hline TotalSeq-A0804 anti-human CD186 (K041E5) & BioLegend & 356021; RRID: AB_2800961 \\
\hline TotalSeq-A0807 anti-mouse CD200R (OX-110) & BioLegend & 123913; RRID: AB_2800594 \\
\hline TotalSeq-A0808 anti-mouse CD193 (J073E5) & BioLegend & 144523; RRID: AB_2800673 \\
\hline TotalSeq-A0809 anti-mouse CD200R3 (Ba13) & BioLegend & 142209; RRID: AB_2800657 \\
\hline TotalSeq-A0810 anti-mouse CD138 (281-2) & BioLegend & 142532; RRID: AB_2800658 \\
\hline TotalSeq-A0811 anti-mouse CD317 (27) & BioLegend & 127027; RRID: AB_2800623 \\
\hline TotalSeq-A0812 anti-mouse CD105 (MJ7/18) & BioLegend & 120421; RRID: AB_2800587 \\
\hline TotalSeq-A0813 anti-mouse CD9 (MZ3) & BioLegend & 124819; RRID: AB_2800600 \\
\hline TotalSeq-A0814 anti-human CD205 (HD30) & BioLegend & 342211; RRID: AB_2800908 \\
\hline TotalSeq-A0816 anti-human CD271 (ME20.4) & BioLegend & 345123; RRID: AB_2814273 \\
\hline TotalSeq-A0817 anti-human CD109 (W7C5) & BioLegend & 323307; RRID: AB_2800848 \\
\hline TotalSeq-A0819 anti-human CD126 (UV4) & BioLegend & 352813; RRID: AB_2800939 \\
\hline TotalSeq-A0821 anti-human CD164 (67D2) & BioLegend & 324809; RRID: AB_2800850 \\
\hline TotalSeq-A0822 anti-human CD142 (NY2) & BioLegend & 365207; RRID: AB_2801007 \\
\hline TotalSeq-A0824 anti-mouse P2X7R (1F11) & BioLegend & 148711; RRID: AB_2800683 \\
\hline TotalSeq-A0825 anti-mouse CD371 (5D3/Clec12a) & BioLegend & 143407; RRID: AB_2800668 \\
\hline TotalSeq-A0826 anti-human CD307c (H5/FcRL3) & BioLegend & 374411; RRID: AB_2801022 \\
\hline TotalSeq-A0827 anti-mouse CD22 (OX-97) & BioLegend & 126113; RRID: AB_2800614 \\
\hline TotalSeq-A0828 anti-human CD307d (413D12) & BioLegend & 340209; RRID: AB_2800902 \\
\hline TotalSeq-A0829 anti-human CD307e (509f6) & BioLegend & 340307; RRID: AB_2800903 \\
\hline TotalSeq-A0830 anti-human CD319 (162.1) & BioLegend & 331821; RRID: AB_2800872 \\
\hline TotalSeq-A0831 anti-human CD138 (DL-101) & BioLegend & 352325; RRID: AB_2800938 \\
\hline TotalSeq-A0834 anti-mouse CD39 (Duha59) & BioLegend & 143813; RRID: AB_2800669 \\
\hline TotalSeq-A0835 anti-mouse CD314 (NKG2D) (CX5) & BioLegend & 130215; RRID: AB_2814023 \\
\hline TotalSeq-A0836 anti-mouse DR3 (4C12) & BioLegend & 144413; RRID: AB_2814048 \\
\hline TotalSeq-A0837 anti-mouse IL33RA (DIH9) & BioLegend & 145317; RRID: AB_2800680 \\
\hline TotalSeq-A0839 anti-mouse Ly49H (3D10) & BioLegend & 144715; RRID: AB_2814049 \\
\hline TotalSeq-A0841 anti-mouse Ly49D (4E5) & BioLegend & 138309; RRID: AB_2800647 \\
\hline TotalSeq-A0843 anti-human CD199 (L053E8) & BioLegend & 358919; RRID: AB_2810569 \\
\hline TotalSeq-A0844 anti-human CD45RB (MEM-55) & BioLegend & 310209; RRID: AB_2810471 \\
\hline TotalSeq-A0845 anti-human CD99 (3B2/TA8) & BioLegend & 371317: RRID: AB_2801017 \\
\hline TotalSeq-A0846 anti-mouse CD185 (L138D7) & BioLegend & 145535; RRID: AB_2800681 \\
\hline TotalSeq-A0848 anti-mouse TIGIT (1G9) & BioLegend & 142115; RRID: AB_2800656 \\
\hline TotalSeq-A0849 anti-mouse CD80 (16-10A1) & BioLegend & 104745; RRID: AB_2813935 \\
\hline TotalSeq-A0850 anti-mouse CD49a (Hma1) & BioLegend & 142613; RRID: AB_2800659 \\
\hline TotalSeq-A0851 anti-mouse CD1d (1B1) & BioLegend & 123529; RRID: AB_2800593 \\
\hline TotalSeq-A0852 anti-mouse CD226 (10E5) & BioLegend & 128823; RRID: AB_2810393 \\
\hline TotalSeq-A0853 anti-human CD371 (50C1) & BioLegend & 353613; RRID: AB_2800948 \\
\hline TotalSeq-A0857 anti-mouse CD34 (HM34) & BioLegend & 128619; RRID: AB_2810392 \\
\hline TotalSeq-A0858 anti-human CD46 (TRA-2-10) & BioLegend & 352415; RRID: AB_2810557 \\
\hline TotalSeq-A0861 anti-human CD151 (50-6) & BioLegend & 350409; RRID: AB_2810554 \\
\hline
\end{tabular}




\begin{tabular}{|c|c|c|}
\hline \multicolumn{3}{|l|}{ Continued } \\
\hline REAGENT or RESOURCE & SOURCE & IDENTIFIER \\
\hline TotalSeq-A0862 anti-human CD218a (H44) & BioLegend & 313815; RRID: AB_2810476 \\
\hline TotalSeq-A0863 anti-human CD257 (1D6) & BioLegend & 366509; RRID: AB_2810575 \\
\hline TotalSeq-A0866 anti-human CLEC1B (AYP1) & BioLegend & 372009; RRID: AB_2814333 \\
\hline TotalSeq-A0867 anti-human CD94 (DX22) & BioLegend & 305521; RRID: AB_2814142 \\
\hline TotalSeq-A0868 anti-human IgE (MHE-18) & BioLegend & 325517; RRID: AB_2814186 \\
\hline TotalSeq-A0869 anti-human CD365 (1D12) & BioLegend & 353907; RRID: AB_2814294 \\
\hline TotalSeq-A0870 anti-human CD150 (A12) & BioLegend & 306313; RRID: AB_2814146 \\
\hline TotalSeq-A0871 anti-human CD162 (KPL-1) & BioLegend & 328821; RRID: AB_2814192 \\
\hline TotalSeq-A0875 anti-mouse TLR4 (MTS510) & BioLegend & 117614; RRID: AB_2810352 \\
\hline TotalSeq-A0876 anti-mouse CD300c_d (TX52) & BioLegend & 148005; RRID: AB_2810413 \\
\hline TotalSeq-A0877 anti-mouse JAML (4E+10) & BioLegend & 128507; RRID: AB_2810391 \\
\hline TotalSeq-A0881 anti-mouse CD272 (6A6) & BioLegend & 139113; RRID: AB_2814041 \\
\hline TotalSeq-A0882 anti-mouse PIRA_PIRB (6C1) & BioLegend & 144105; RRID: AB_2810412 \\
\hline TotalSeq-A0883 anti-mouse CD26 (H194-112) & BioLegend & 137811; RRID: AB_2810405 \\
\hline TotalSeq-A0884 anti-mouse DLL1 (HMD1-3) & BioLegend & 128315; RRID: AB_2810390 \\
\hline TotalSeq-A0885 anti-mouse CD270 (HMHC-1B18) & BioLegend & 136307; RRID: AB_2810403 \\
\hline TotalSeq-A0890 anti-mouse 4_1BB (TKS-1) & BioLegend & 107109: RRID: AB_2813955 \\
\hline TotalSeq-A0891 anti-mouse ENPP (YE1/19.1) & BioLegend & 149209; RRID: AB_2814052 \\
\hline TotalSeq-A0892 anti-mouse CD2 (RM2-5) & BioLegend & 100117; RRID: AB_2810312 \\
\hline TotalSeq-A0894 anti-human Ig (MHL-38) & BioLegend & 316531; RRID: AB_2810479 \\
\hline TotalSeq-A0895 anti-mouse/human Mac2 (LM3/38) & BioLegend & 125421; RRID: AB_281384 \\
\hline TotalSeq-A0896 anti-human CD85j (GHI/75) & BioLegend & 333723; RRID: AB_2814225 \\
\hline TotalSeq-A0897 anti-human CD23 (EBVCS-5) & BioLegend & 338523; RRID: AB_2814235 \\
\hline TotalSeq-A0898 anti-human Ig (MHK-49) & BioLegend & 316627; RRID: AB_2814170 \\
\hline TotalSeq-A0899 anti-human HLA-A2 (BB7.2) & BioLegend & 343331; RRID: AB_2810540 \\
\hline TotalSeq-A0900 anti-human CD198 (L263G8) & BioLegend & 360607; RRID: AB_2810572 \\
\hline TotalSeq-A0901 anti-human GARP (7B11) & BioLegend & 352515; RRID: AB_2814283 \\
\hline TotalSeq-A0904 anti-mouse CD31 (390) & BioLegend & 102437; RRID: AB_2810335 \\
\hline TotalSeq-A0905 anti-mouse CD107a (1D4B) & BioLegend & 121635; RRID: AB_2810369 \\
\hline TotalSeq-A0916 anti-mouse CD124 (I015F8) & BioLegend & 144809; RRID: AB_2814050 \\
\hline TotalSeq-A0917 anti-mouse CD95 (SA367H8) & BioLegend & 152614; RRID: AB_2810418 \\
\hline TotalSeq-A0923 anti-human NKp80 (5D12) & BioLegend & 346709; RRID: AB_2814274 \\
\hline TotalSeq-A5106 anti-mouse Ly6D & BioLegend & Barcode: ATGTCCTACCTCAAA \\
\hline \multicolumn{3}{|l|}{ Chemicals, peptides, and recombinant proteins } \\
\hline Antigenfix & Diapath & P0014 \\
\hline$\beta$-mercaptoethanol & Sigma-Aldrich & M3148 \\
\hline Calcium chloride dihydrate & Merck & 1023821000 \\
\hline Collagenase A & Sigma-Aldrich & 11088793001 \\
\hline D-(-)-Fructose & Merck-Millipore & 1040071000 \\
\hline $\mathrm{D}(+)$-Saccharose & VWR International & PROL27483.294 \\
\hline DAPI & Invitrogen & D1306; RIDD: AB_2629482 \\
\hline DMEM & Invitrogen & $41965-039$ \\
\hline Dnase I & Sigma-Aldrich & 04536282001 \\
\hline Donkey Serum & Abcam & $a b 7475$ \\
\hline EDTA & Westburg & 51234 \\
\hline EGTA & Sigma-Aldrich & E3889 \\
\hline Eosin & VWR International & MERC 1.15935 \\
\hline FcBlock 2.4G2 & Bioceros & N/A \\
\hline FCS & Bodinco & 5010 \\
\hline
\end{tabular}




\begin{tabular}{|c|c|c|}
\hline \multicolumn{3}{|l|}{ Continued } \\
\hline REAGENT or RESOURCE & SOURCE & IDENTIFIER \\
\hline Fixable Viability due Live/Dead - eFluor506 & eBioscience & $65-0866-18$ \\
\hline Fixable Viability due Live/Dead - eFluor780 & eBioscience & $65-0865-18$ \\
\hline Fixation/Permeabilization Solution Kit & BD Cytofix/Cytoperm & 554714 \\
\hline FoxP3 Transcription factor staining buffer kit & eBioscience & $00-5523-00$ \\
\hline Gentamicin & Gibco & $15710-049$ \\
\hline GlutaMAX & Thermo Fisher & $35050-038$ \\
\hline Gluteraldehyde $25 \%$ & Sigma-Aldrich & G5882 \\
\hline Goat Serum & Sigma-Aldrich & G9023 \\
\hline Hematoxylin & VWR International & MERC1.05174 \\
\hline HEPES & Sigma-Aldrich & H3375 \\
\hline Isopropanol & Vel & T0108 \\
\hline Methanol & Merck Millipore & 13680502 \\
\hline Paraformaldehyde $10 \%$ & EMS & 15712 \\
\hline Phenol Red & Sigma-Aldrich & P3532 \\
\hline ProLong Diamond & Thermo Fisher & P36970 \\
\hline Rat Serum & Sigma-Aldrich & R9759 \\
\hline Ribonucleoside Vanadyl Complexes & Sigma-Aldrich & R3380-5ML \\
\hline Roche Protector RNAse inhibitor & Sigma-Aldrich & 3335399001 \\
\hline RPMI 1640 & Gibco & $52400-025$ \\
\hline Saponin & Sigma-Aldrich & 4521 \\
\hline $10 \times$ SDS & Sigma-Aldrich & 71736-100ML \\
\hline Sodium bicarbonate & Sigma-Aldrich & 792519 \\
\hline Sodium chloride & Sigma-Aldrich & 746398 \\
\hline Sodium Dihydrogen Phosphate Monohydrate & Sigma-Aldrich & 1063461000 \\
\hline Sodium phosphate dibasic dihydrate & Sigma-Aldrich & 71643 \\
\hline Tissue-Tek O.C.T & Sakura Finetek & 4583 \\
\hline UltraPure ${ }^{\mathrm{TM}}$ Salmon Sperm DNA Solution & Thermo Fisher Scientific & 15632011 \\
\hline Xylene & Prolabo & PROL28973.363 \\
\hline \multicolumn{3}{|l|}{ Critical commercial assays } \\
\hline ALLin HS Red Taq Mastermix $2 x$ & highQu & HQ.HSM0350 \\
\hline RNEasy Plus Micro Kit & QIAGEN & 74034 \\
\hline SensiFAST cDNA Synthesis Kit & Bioline & $\mathrm{BIO}-65054$ \\
\hline SensiFAST SYBR No-ROX Kit & Bioline & BIO-98020 \\
\hline Visium Spatial Gene Expression Slide and Reagent Kit & 10X Genomics & 1000184 \\
\hline \multicolumn{3}{|l|}{ Oligonucleotides: qPCR } \\
\hline Flt3 - qPCR FWD & IDT & GTGACTGGCCCCCTGGATAACGAG \\
\hline Flt3 - qPCR REV & IDT & TCCAAGGGCGGGTGTAACTGAACT \\
\hline Xcr1 - qPCR FWD & IDT & AGAGACACCGAACAGTCAGGCT \\
\hline Xcr1 - qPCR REV & IDT & TGTCCAGTTGCTGAAGGCTCTC \\
\hline Cd209a - qPCR FWD & IDT & GCACTCCATCAAAGGCTTTGGC \\
\hline Cd209a - qPCR REV & IDT & CAAACAGCTAGGAAGAGCACCTG \\
\hline Ccr7 - qPCR FWD & IDT & AGAGGCTCAAGACCATGACGGA \\
\hline Ccr7 - qPCR REV & IDT & TCCAGGACTTGGCTTCGCTGTA \\
\hline Mafb - qPCR FWD & IDT & TGAATTTGCTGGCACTGCTG \\
\hline Mafb - qPCR REV & IDT & AAGCACCATGCGGTTCATACA \\
\hline Cd5I - qPCR FWD & IDT & GAGGACACATGGATGGAATGT \\
\hline Cd5I - qPCR REV & IDT & ACCCTTGTGTAGCACCTCCA \\
\hline
\end{tabular}




\begin{tabular}{|c|c|c|}
\hline \multicolumn{3}{|l|}{ Continued } \\
\hline REAGENT or RESOURCE & SOURCE & IDENTIFIER \\
\hline Cd207 - qPCR FWD & IDT & CCGAAGCGCACTTCACAGT \\
\hline Cd207 - qPCR REV & IDT & GCAGATACAGAGAGGTTTCCTCA \\
\hline Spp1 - qPCR FWD & IDT & ССАTCTCAGAAGCAGAATCTCCTT \\
\hline Spp1 - qPCR REV & IDT & GGTCATGGCTTTCATTGGAATT \\
\hline Gata6 - qPCR FWD & IDT & CAGCAGGACCCTTCGAAAC \\
\hline Gata6 - qPCR REV & IDT & CCATTCATCTTGCTGTAGAGACC \\
\hline Gpnmb - qPCR FWD & IDT & AGCACAACCAATTACGTGGC \\
\hline Gpnmb - qPCR REV & IDT & CTTCCCAGGAGTCCTTCCA \\
\hline Pla2g7 - qPCR FWD & IDT & CTTCAAGCCCTTAGTGAGGACC \\
\hline Pla2g7 - qPCR REV & IDT & TGCGATGTCCTTTGGAGTCTGG \\
\hline Cd9 - qPCR FWD & IDT & $\begin{array}{l}\text { GCTACTCGAGCCATGCCGGTCA } \\
\text { AAGGAGGTAGC }\end{array}$ \\
\hline Cd9 - qPCR REV & IDT & $\begin{array}{l}\text { CACTTGGTACCGACCATTTCTC } \\
\text { GGCTCCTGCG }\end{array}$ \\
\hline Cd36 - qPCR FWD & IDT & GGAGCCATCTTTGAGCCTTCA \\
\hline Cd36 - qPCR REV & IDT & GAACCAAACTGAGGAATGGATCT \\
\hline$\| / 1 b-q P C R$ FWD & IDT & GCAACTGTTCCTGAACTCAACT \\
\hline II1b - qPCR REV & IDT & ATCTITTGGGGTCCGTCAACT \\
\hline Tnf - qPCR FWD & IDT & TCTTCTCATTCCTGCTTGTGG \\
\hline Tnf - qPCR REV & IDT & GGTCTGGGCCATAGAACTGA \\
\hline II10 - qPCR FWD & IDT & AAGGCAGTGGAGCAGGTGAA \\
\hline II10 - qPCR REV & IDT & CCAGCAGACTCAATACACAC \\
\hline II18 - qPCR FWD & IDT & ACTGTACAACCGCAGTAATACGC \\
\hline II18 - qPCR REV & IDT & AGTGAACATTACAGATTTATCCC \\
\hline Actb - qPCR FWD & IDT & GCTTCTAGGCGGACTGTTACTGA \\
\hline Actb - qPCR REV & IDT & GCCATGCCAATGTTGTCTCTTAT \\
\hline \multicolumn{3}{|c|}{ Oligonucleotides: Molecular cartography probes } \\
\hline FLT3 & Resolve Bio. & P0C6W \\
\hline PDGFRB & Resolve Bio. & P0D6V \\
\hline LGR5 & Resolve Bio. & P0E6T \\
\hline OTOA & Resolve Bio. & PON7M \\
\hline CLEC10A & Resolve Bio. & P1C6X \\
\hline COLEC11 & Resolve Bio. & P1D6W \\
\hline GLS2 & Resolve Bio. & P1E6V \\
\hline KRT19 & Resolve Bio. & P1N6M \\
\hline$S D S$ & Resolve Bio. & P1N7N \\
\hline SIRPA & Resolve Bio. & P2C6Y \\
\hline NGFR & Resolve Bio. & P2D6X \\
\hline$H A L$ & Resolve Bio. & P2E6W \\
\hline GNLY & Resolve Bio. & P2K1J \\
\hline SLC16A9 & Resolve Bio. & P2N7P \\
\hline CD1E & Resolve Bio. & P3C6Z \\
\hline$H G F$ & Resolve Bio. & P3D6Y \\
\hline SLC38A1 & Resolve Bio. & P3N7Q \\
\hline ITGAX & Resolve Bio. & P4422 \\
\hline$C D 4$ & Resolve Bio. & P4A2X \\
\hline ADAMTSL2 & Resolve Bio. & P4D6Z \\
\hline SLC4OA1 & Resolve Bio. & P4N7R \\
\hline CDKN1C & Resolve Bio. & P5A1X \\
\hline
\end{tabular}




\begin{tabular}{|c|c|c|}
\hline \multicolumn{3}{|l|}{ Continued } \\
\hline REAGENT or RESOURCE & SOURCE & IDENTIFIER \\
\hline СРАЗ & Resolve Bio. & P5C60 \\
\hline MFAP4 & Resolve Bio. & P5D6L \\
\hline CXCR6 & Resolve Bio. & P6C61 \\
\hline COL1A1 & Resolve Bio. & P6D60 \\
\hline$C D 3 E$ & Resolve Bio. & P6J1Q \\
\hline TIMD4 & Resolve Bio. & P6N7T \\
\hline NCR1 & Resolve Bio. & P7A2L \\
\hline CXCL12 & Resolve Bio. & P7C62 \\
\hline MARCO & Resolve Bio. & P8C63 \\
\hline MYH11 & Resolve Bio. & P8D62 \\
\hline$C D 5 L$ & Resolve Bio. & P9C64 \\
\hline ACTA2 & Resolve Bio. & P9D63 \\
\hline IGFBP3 & Resolve Bio. & PAA11 \\
\hline VSIG4 & Resolve Bio. & PAC65 \\
\hline F8 & Resolve Bio. & PAD64 \\
\hline NKG7 & Resolve Bio. & PC13E \\
\hline GPNMB & Resolve Bio. & PCC66 \\
\hline CMTM2 & Resolve Bio. & PDC67 \\
\hline FLT4 & Resolve Bio. & PDD66 \\
\hline CTSG & Resolve Bio. & PDN7L \\
\hline CSF1R & Resolve Bio. & PDR2R \\
\hline$C D 79 A$ & Resolve Bio. & PE13G \\
\hline EPCAM & Resolve Bio. & PEW3P \\
\hline FCGR3B & Resolve Bio. & PFC69 \\
\hline FCGR3A & Resolve Bio. & PFT1Q \\
\hline FOLR2 & Resolve Bio. & PFW6T \\
\hline LILRA4 & Resolve Bio. & PGC6A \\
\hline FCGBP & Resolve Bio. & PGN61 \\
\hline PLA2G7 & Resolve Bio. & PGN72 \\
\hline$D P T$ & Resolve Bio. & PGX6T \\
\hline CLEC4C & Resolve Bio. & PHC6C \\
\hline WNT2 & Resolve Bio. & PHD6A \\
\hline$S P N$ & Resolve Bio. & PHM1Z \\
\hline LHX6 & Resolve Bio. & PJD6C \\
\hline$C D 8 A$ & Resolve Bio. & PJJ11 \\
\hline$A X L$ & Resolve Bio. & PJR2X \\
\hline$V W F$ & Resolve Bio. & PKD6D \\
\hline$D C N$ & Resolve Bio. & PKY4T \\
\hline FCN1 & Resolve Bio. & PMC6F \\
\hline RSPO3 & Resolve Bio. & PMD6E \\
\hline CCR7 & Resolve Bio. & PMH14 \\
\hline HTRA3 & Resolve Bio. & PMJ68 \\
\hline CCL21 & Resolve Bio. & PMM77 \\
\hline CLEC12A & Resolve Bio. & PNC6G \\
\hline GJA5 & Resolve Bio. & PND6F \\
\hline STAB2 & Resolve Bio. & PNJ69 \\
\hline$C D 36$ & Resolve Bio. & PNM78 \\
\hline$C D 68$ & Resolve Bio. & PPC6H \\
\hline LYVE1 & Resolve Bio. & PPJ6A \\
\hline
\end{tabular}




\begin{tabular}{|c|c|c|}
\hline \multicolumn{3}{|l|}{ Continued } \\
\hline REAGENT or RESOURCE & SOURCE & IDENTIFIER \\
\hline IL7R & Resolve Bio. & PPK14 \\
\hline$C D 9$ & Resolve Bio. & PPM79 \\
\hline MSLN & Resolve Bio. & PQJ6C \\
\hline ENHO & Resolve Bio. & PQM7A \\
\hline TREM2 & Resolve Bio. & PR32Q \\
\hline sox9 & Resolve Bio. & PRD6J \\
\hline RELN & Resolve Bio. & PRM15 \\
\hline$F 13 A 1$ & Resolve Bio. & PRM7C \\
\hline SPP1 & Resolve Bio. & PSD6K \\
\hline FCER1A & Resolve Bio. & PSM7D \\
\hline MAFB & Resolve Bio. & PTA6P \\
\hline CD19 & Resolve Bio. & PTH1A \\
\hline FCGR1A & Resolve Bio. & PTM7E \\
\hline C5AR1 & Resolve Bio. & PVA6Q \\
\hline GDF15 & Resolve Bio. & PVM7F \\
\hline$C D 1 C$ & Resolve Bio. & PWA6R \\
\hline FCGR2B & Resolve Bio. & PWC2K \\
\hline WT1 & Resolve Bio. & PWD6P \\
\hline GLUL & Resolve Bio. & PWG1E \\
\hline IGSF21 & Resolve Bio. & PWM7G \\
\hline XCR1 & Resolve Bio. & PXA6S \\
\hline CYP2E1 & Resolve Bio. & PXD6Q \\
\hline$C D 14$ & Resolve Bio. & PXS37 \\
\hline CLEC9A & Resolve Bio. & PYA6T \\
\hline LILRB5 & Resolve Bio. & PYM7J \\
\hline THBD & Resolve Bio. & PZA6V \\
\hline$D E S$ & Resolve Bio. & PZC6T \\
\hline GHR & Resolve Bio. & PZD6S \\
\hline NDST3 & Resolve Bio. & PZM7K \\
\hline Trem2 & Resolve Bio. & P0E2P \\
\hline Wt1 & Resolve Bio. & P0E4R \\
\hline Adgrg6 & Resolve Bio. & POF4Q \\
\hline Lilra5 & Resolve Bio. & P0M7N \\
\hline $\operatorname{ltgax}$ & Resolve Bio. & P1C1R \\
\hline Dcn & Resolve Bio. & P1E4S \\
\hline Mmrn1 & Resolve Bio. & P1F4R \\
\hline$L p l$ & Resolve Bio. & P1M7P \\
\hline$C d 79 a$ & Resolve Bio. & P2662 \\
\hline Cd36 & Resolve Bio. & P2A5Y \\
\hline Gpnmb & Resolve Bio. & $\mathrm{P} 2 \mathrm{H} 7 \mathrm{~T}$ \\
\hline Marco & Resolve Bio. & P2M7Q \\
\hline Cd19 & Resolve Bio. & P3663 \\
\hline Pdgfrb & Resolve Bio. & P3E4V \\
\hline Adgre1 & Resolve Bio. & P3F4T \\
\hline Adamts/2 & Resolve Bio. & P3K7S \\
\hline Mfap4 & Resolve Bio. & P3M7R \\
\hline Lyve1 & Resolve Bio. & P4961 \\
\hline$A \times I$ & Resolve Bio. & P4C1V \\
\hline Olfm/3 & Resolve Bio. & P4E2T \\
\hline Atp6v0d2 & Resolve Bio. & $\mathrm{P} 4 \mathrm{~K} 7 \mathrm{~T}$ \\
\hline
\end{tabular}




\begin{tabular}{|c|c|c|}
\hline \multicolumn{3}{|l|}{ Continued } \\
\hline REAGENT or RESOURCE & SOURCE & IDENTIFIER \\
\hline Mmp12 & Resolve Bio. & P4M7S \\
\hline C5ar1 & Resolve Bio. & P5K7V \\
\hline Msln & Resolve Bio. & P5M7T \\
\hline Lhx6 & Resolve Bio. & P5T2F \\
\hline Fn1 & Resolve Bio. & P6952 \\
\hline Ccr7 & Resolve Bio. & P6K7W \\
\hline Cd14 & Resolve Bio. & P7K7X \\
\hline Nrxn1 & Resolve Bio. & P7M7W \\
\hline Acta2 & Resolve Bio. & P8668 \\
\hline Cd2O7 & Resolve Bio. & P8K7Y \\
\hline$C d 5 /$ & Resolve Bio. & P9K7Z \\
\hline Rspo3 & Resolve Bio. & P9M7Y \\
\hline Ccr2 & Resolve Bio. & PAF40 \\
\hline Slc40a1 & Resolve Bio. & PAM7Z \\
\hline Gls2 & Resolve Bio. & PAN7Y \\
\hline Sds & Resolve Bio. & PCG40 \\
\hline Clec10a & Resolve Bio. & PCK70 \\
\hline Spon2 & Resolve Bio. & PCM7L \\
\hline Pck1 & Resolve Bio. & PCN7Z \\
\hline Sirpa & Resolve Bio. & $\mathrm{PDH} 73$ \\
\hline Clec4f & Resolve Bio. & PDK71 \\
\hline Stab2 & Resolve Bio. & PDM70 \\
\hline Krt19 & Resolve Bio. & PE07N \\
\hline Siglech & Resolve Bio. & PEF43 \\
\hline Clic5 & Resolve Bio. & PEK72 \\
\hline Svep1 & Resolve Bio. & PEM71 \\
\hline$C d 9$ & Resolve Bio. & PFH75 \\
\hline Col1a1 & Resolve Bio. & PFK73 \\
\hline Timd4 & Resolve Bio. & PFM72 \\
\hline Pecam1 & Resolve Bio. & PFP5Z \\
\hline$C \times 3 c r 1$ & Resolve Bio. & PG35J \\
\hline Ncam1 & Resolve Bio. & PGE46 \\
\hline Colec11 & Resolve Bio. & PGK74 \\
\hline Tmem119 & Resolve Bio. & PGM73 \\
\hline Pdgfra & Resolve Bio. & PHG45 \\
\hline Cox6a2 & Resolve Bio. & PHK75 \\
\hline Upk3b & Resolve Bio. & PHM74 \\
\hline Cxc/12 & Resolve Bio. & PJK76 \\
\hline Wnt2 & Resolve Bio. & PJM75 \\
\hline Reln & Resolve Bio. & PK54J \\
\hline Fcgr1 & Resolve Bio. & PK86H \\
\hline Myh11 & Resolve Bio. & PK95F \\
\hline Des & Resolve Bio. & PKK77 \\
\hline Wnt9b & Resolve Bio. & PKM76 \\
\hline Sox9 & Resolve Bio. & PKT6L \\
\hline Chil3 & Resolve Bio. & PMH7A \\
\hline$D p t$ & Resolve Bio. & PMK78 \\
\hline Grip1 & Resolve Bio. & PND2A \\
\hline Ms4a7 & Resolve Bio. & $\mathrm{PNH7C}$ \\
\hline
\end{tabular}




\begin{tabular}{|c|c|c|}
\hline \multicolumn{3}{|l|}{ Continued } \\
\hline REAGENT or RESOURCE & SOURCE & IDENTIFIER \\
\hline F13a1 & Resolve Bio. & PNK79 \\
\hline Vsig4 & Resolve Bio. & PPH7D \\
\hline Flt3 & Resolve Bio. & PPK7A \\
\hline Spp1 & Resolve Bio. & PQD4F \\
\hline Flt4 & Resolve Bio. & PQK7C \\
\hline Epcam & Resolve Bio. & PRD4G \\
\hline Plpp1 & Resolve Bio. & PRE4F \\
\hline Folr2 & Resolve Bio. & PRK7D \\
\hline Cd3e & Resolve Bio. & PS56T \\
\hline Clec9a & Resolve Bio. & PS66S \\
\hline Ghr & Resolve Bio. & PSF4F \\
\hline Mafb & Resolve Bio. & PSS66 \\
\hline Gja5 & Resolve Bio. & PTK7F \\
\hline Сyp2e1 & Resolve Bio. & PVF4H \\
\hline Sept3 & Resolve Bio. & PW66W \\
\hline$V w f$ & Resolve Bio. & PWE4K \\
\hline Lgr5 & Resolve Bio. & PWF4J \\
\hline $\mathrm{Hal}$ & Resolve Bio. & PWK7H \\
\hline Prox1 & Resolve Bio. & PWS25 \\
\hline Xcr1 & Resolve Bio. & PX86V \\
\hline$H g f$ & Resolve Bio. & PXK7J \\
\hline Itgae & Resolve Bio. & PY86W \\
\hline Spn & Resolve Bio. & PYE7R \\
\hline Igfbp3 & Resolve Bio. & PYK7K \\
\hline Cd209a & Resolve Bio. & PYM6H \\
\hline Glul & Resolve Bio. & PZ81R \\
\hline $\operatorname{ltg} b 7$ & Resolve Bio. & PZK7M \\
\hline Mgl2 & Resolve Bio. & PZM6J \\
\hline \multicolumn{3}{|l|}{ Software and algorithms } \\
\hline Adobe Illustrator & Adobe & www.adobe.com \\
\hline Cell Ranger & 10X Genomics & $\begin{array}{l}\text { https://support.10xgenomics.com/single- } \\
\text { cell-gene-expression/software/pipelines/ } \\
\text { latest/what-is-cell-ranger }\end{array}$ \\
\hline FastCAR R Package & N/A & https://github.com/LungCellAtlas/FastCAR \\
\hline Seurat R Package V3 & (Stuart et al., 2019) & https://satijalab.org/seurat/ \\
\hline Scater R Package & (McCarthy et al., 2017) & $\begin{array}{l}\text { https://bioconductor.org/packages/release/ } \\
\text { bioc/html/scater.html }\end{array}$ \\
\hline BioMart & $\mathrm{N} / \mathrm{A}$ & $\begin{array}{l}\text { https://www.ensembl.org/biomart/martview/ } \\
\text { 3e2c65a5e3f783f8c9e5d648e4b64126 }\end{array}$ \\
\hline pheatmap R package & $\mathrm{N} / \mathrm{A}$ & https://rdrr.io/cran/pheatmap/ \\
\hline ggplot2 & (Wickham 2016) & https://ggplot2.tidyverse.org \\
\hline Scanpy & (Wolf et al., 2018) & https://scanpy.readthedocs.io/en/stable/ \\
\hline PyTorch & $\mathrm{N} / \mathrm{A}$ & https://pytorch.org \\
\hline TotalVI & (Gayoso et al., 2021) & $\begin{array}{l}\text { https://docs.scvi-tools.org/en/stable/ } \\
\text { user_guide/models/totalvi.html }\end{array}$ \\
\hline ScVI & (Lopez et al., 2018) & $\begin{array}{l}\text { https://docs.scvi-tools.org/en/stable/ } \\
\text { user_guide/models/totalvi.html }\end{array}$ \\
\hline NicheNet & (Browaeys et al. 2019) & https://github.com/saeyslab/nichenetr \\
\hline Enrichr & (Kuleshov et al., 2016) & http://amp.pharm.mssm.edu/Enrichr/ \\
\hline FlowJo v10.6.1 & FlowJo & https://www.flowjo.com \\
\hline
\end{tabular}




\begin{tabular}{|c|c|c|}
\hline \multicolumn{3}{|l|}{ Continued } \\
\hline REAGENT or RESOURCE & SOURCE & IDENTIFIER \\
\hline GeneOntology & $\begin{array}{l}\text { (Ashburner et al., } \\
\text { 2000) }\end{array}$ & http://geneontology.org/ \\
\hline GraphPad Prism 9 & GraphPad & https://www.graphpad.com/ \\
\hline Harmony & (Korsunsky et al., 2019) & $\begin{array}{l}\text { https://www.github.com/ } \\
\text { immunogenomics/harmony }\end{array}$ \\
\hline llastik & (Berg et al., 2019) & https://www.ilastik.org/ \\
\hline ImageJ & (Schneider et al., 2012) & https://imagej.nih.gov/ij \\
\hline Qi-Tissue & $\begin{array}{l}\text { Quantitative Imagining } \\
\text { Systems }\end{array}$ & https://www.qi-tissue.com \\
\hline genexyz Polylux & Resolve Biosciences & \\
\hline Single-Cell Signature Explorer algorithm & (Pont et al., 2019) & $\begin{array}{l}\text { https://sites.google.com/site/ } \\
\text { fredsoftwares/products/single- } \\
\text { cell-signature-explorer }\end{array}$ \\
\hline WEB-based GEne SeT AnaLysis Toolkit & (Liao et al., 2019) & http://www.webgestalt.org/ \\
\hline Venn Diagram Generator & PSB, VIB-Ghent University & $\begin{array}{l}\text { https://bioinformatics.psb. } \\
\text { ugent.be/webtools/Venn/ }\end{array}$ \\
\hline Zen Black & ZEISS Microscopy & www.zeiss.com \\
\hline \multicolumn{3}{|l|}{ Experimental models } \\
\hline Mouse: B6(C)-Ccr2 ${ }^{\operatorname{tm} 1.1 \mathrm{Cln} / \mathrm{J}}$ & The Jackson Laboratory & JAX: 027619 \\
\hline Mouse: C57BL/6j SPF & Janvier Labs & $\mathrm{N} / \mathrm{A}$ \\
\hline Mouse: B6-Clec4f ${ }^{\text {hDTR/YFP-CIPHE }}$ & (Scott et al., 2016) & $\mathrm{N} / \mathrm{A}$ \\
\hline Mouse: B6-Clec4f ${ }^{\text {Cre-CIPHE }}$ & (Scott et al., 2018) & N/A \\
\hline Mouse: Alk1-flox & (Park et al., 2008) & $\mathrm{N} / \mathrm{A}$ \\
\hline
\end{tabular}

\section{RESOURCE AVAILABILITY}

\section{Lead contact}

Further information and requests for resources and reagents should be directed to and will be fulfilled by the lead contact, Martin Guilliams (martin.guilliams@irc.vib-ugent.be).

\section{Materials availability}

This study did not generate new unique reagents.

Data and code availability

The datasets generated during this study have been deposited in the Gene Expression Omnibus public database under accession number GSE192742.

\section{EXPERIMENTAL MODEL AND SUBJECT DETAILS}

\section{In vivo animal studies}

Mice

WT C57BI/6J mice (Janvier) were used for this study. Male and Female mice were used for all experiments, with the exception of NAFLD experiments were only male mice were used. For NAFLD experiments, mice were put on the SD or WD at 5 weeks of age and sacrificed after either 24 or 36 weeks on the diet as indicated. Fcgr1-Cre mice (Scott et al., 2018) were obtained from Prof. Bernard Malissen, CIML, Marseille and Clec4f-Cre mice (Scott et al., 2018) were crossed with Acvrl $1^{\mathrm{fl} / \mathrm{fl}}$ mice (Park et al., 2008) obtained from Paul Oh, Barrow Neurological Institute, Florida, USA. Clec4f-Dtr mice (Scott et al., 2016) were crossed to CD45.1 mice (Janvier) for $\mathrm{KC}$ depletion and development experiments All mice were used between 6 and 12 weeks of age unless otherwise stated. All mice maintained at the VIB (Ghent University) under specific pathogen free conditions. All animals were randomly allocated to experimental groups. All experiments were performed in accordance with the ethical committee of the Faculty of Science, UGent and VIB. 


\section{Pig}

Piglets (female, 10 weeks old) were purchased at a local farm and transported to the animal facilities of the Faculty of Veterinary Medicine. The animals were housed in isolation units as blood donors and had access to feed and water ad libitum. At 30 weeks of age, the animals were euthanized by intravenous injection of sodium pentobarbital $20 \%(60 \mathrm{mg} / 2.5 \mathrm{~kg})$ and livers were collected. The animal study was reviewed and approved by the Ethical Committee of the Faculty of Veterinary Medicine (EC2018/55).

Chicken

Study animals were clinically healthy Leghorn hens of approximately 58 weeks old collected from a commercial farm. The hens were housed at the Faculty of Veterinary Medicine according to acceptable welfare standards and were observed at least twice daily for health problems. Feed and water was offered ad libitum. The chickens were euthanized through intravenous injection (in the wing) with sodium pentobarbital. The EC approval number of this trial was EC2019/015.

\section{Macaque}

Male Cynomolgus macaques ( $\geq 2$ years) were sourced from China and supplied by Guangzhou Xiangguan Biotech Co., Ltd and confirmed healthy before being assigned to the study. Animal handling, husbandry and euthanasia was performed by WuxiAppTec Co., Ltd., China according to local ethical guidelines (AAALAC accredited 2010). Study animals consists of 4 groups, orally dosed once with a Janssen proprietary immune modulator or vehicle control. Vehicle control animals only were used in this study. Liver tissue samples were snap-frozen immediately after euthanasia. Samples were thawed once before shipping to Ghent for snRNA-seq analysis.

\section{Hamster}

Female syrian hamsters (Janvier) were housed per one or two in ventilated isolator cages at a temperature of $21^{\circ} \mathrm{C}$, humidity of $55 \%$ and 12:12 dark/light cycles, with access to food and water ad libitum and cage enrichment. All hamsters had SPF status at arrival and manipulations were performed in a laminar flow cabinet. Housing conditions and experimental procedures were approved by the ethical committee of KU Leuven (license P015-2020). Animals were euthanized at 6-8 weeks of age by intraperitoneal injection of $200 \mathrm{mg} / \mathrm{mL}$ sodium pentobarbital and livers were collected for analysis.

Zebrafish

Zebrafish were maintained under standard conditions, according to FELASA guidelines (Alestrom et al., 2019). All experimental procedures were approved by the ethical committee for animal welfare (CEBEA) from the ULB (Université Libre de Bruxelles) (Protocol \#594N). The following transgenic lines at 6 months of age were used: $T g$ (mpeg1:EGFP)gl22 (Ellett et al., 2011); Tg(kdrl:Cre) ${ }^{\text {s89 }}$ (Bertrand et al., 2010); Tg(actb2:IoxP-STOP-loxP-DsRed $\left.{ }^{\text {express}}\right)^{\text {sd5 }}$ (Bertrand et al., 2010) enabling macs to be sorted for sequencing as DsRed, GFP double positive cells.

\section{Patient studies}

Patient studies were run in collaboration with Ghent University Hospital. Liver biopsies $\left(1-2 \mathrm{~mm}^{3}\right)$ were isolated with informed consent from patients undergoing cholecystectomy or gastric bypass. In addition, liver biopsies were isolated from healthy adjacent tissue removed during liver resection due to colorectal cancer metastasis. In most cases, a second biopsy was also taken to evaluate liver histology. A full overview of all patient samples used in this study can be found in Table S6. Paraffin-embedded human liver samples were obtained through collaboration with Dr. Jan Lerut (Université Catholique de Louvain, UCL). All studies were performed in accordance with the ethical committee of the Ghent University Hospital (study numbers: 2015/1334 and 2017/0539).

\section{METHOD DETAILS}

\section{Isolation of liver cells}

Liver cells were isolated by either ex vivo digestion (all species, except zebrafish) or in vivo liver perfusion (mice only) and digestion as described previously (Bonnardel et al., 2019; Scott et al., 2016). Briefly, for ex vivo digestion, livers were isolated, cut into small pieces and incubated with $1 \mathrm{mg} / \mathrm{ml}$ Collagenase $A$ and $10 \mathrm{U} / \mathrm{ml}$ DNAse at $37^{\circ} \mathrm{C}$ for 20 mins with shaking. For in vivo digestion, after retrograde cannulation, livers were perfused for $1-2 \mathrm{mins}$ with an EGTA-containing solution, followed by a $5 \mathrm{~min}(6 \mathrm{ml} / \mathrm{min})$ perfusion with $0.2 \mathrm{mg} /$ $\mathrm{ml}$ collagenase A. Livers were then removed, minced and incubated for $20 \mathrm{mins}$ with $0.4 \mathrm{mg} / \mathrm{ml}$ collagenase A and $10 \mathrm{U} / \mathrm{ml} \mathrm{DNase}$ at $37^{\circ} \mathrm{C}$. All subsequent procedures were performed at $4^{\circ} \mathrm{C}$. Samples were filtered over a $100 \mu \mathrm{m}$ mesh filter and red blood cells were lysed. Samples were again filtered over a $40 \mu \mathrm{m}$ mesh filter. At this point in vivo digestion samples only were subjected to two centrifugation steps of $1 \mathrm{~min}$ at $50 \mathrm{~g}$ to isolate hepatocytes. Remaining liver cells (leukocytes, LSECs and HSCs; in vivo protocol) and total cells from the ex vivo digests were centrifuged at $400 \mathrm{~g}$ for $5 \mathrm{mins}$ before proceeding to antibody staining for flow cytometry. A combination of Collagenase $A$ and DNase were used to digest livers in both protocols to minimize cleavage of surface epitopes.

Dissected livers from 6 months old transgenic zebrafish were triturated and treated with Liberase TM at $33^{\circ} \mathrm{C}$ for 20 min. Cells were then filtered through $40 \mu$ m nylon mesh and washed with $2 \%$ FBS in PBS by centrifugation. Sytox Red was then added to the samples at a final concentration of $5 \mathrm{nM}$ to exclude nonviable cells before proceeding to flow cytometry. DsRed ${ }^{+} \mathrm{GFP}^{+}$cells were then FACS-purified.

Isolation of liver nuclei

Nuclei were isolated from snap frozen liver tissue with a sucrose gradient as previously described (Habib et al., 2016). Briefly, frozen liver tissue is homogenized using Kimble Douncer grinder set in $1 \mathrm{ml}$ homogenization buffer with RNAse inhibitors. Homogenised 
tissue is then is then subjected to density gradient (29\% cushion - Optiprep) ultracentrifugation (7700rpm, $\left.4^{\circ} \mathrm{C}, 30 \mathrm{mins}\right)$. After resuspension, nuclei are stained with DAPI and intact nuclei were FACS-purified from remaining debris.

\section{Diet induced murine model of NAFLD/NASH}

To induce NAFLD and NASH, mice were fed a western diet (WD) high in fat, sugar and cholesterol for 24 or 36 weeks as described previously (Remmerie et al., 2020). This consisted of $58 \%$ fat, $1 \%$ cholesterol (Research Diets; D09061703i) and drinking water was supplemented with $23.1 \mathrm{~g} / \mathrm{L}$ fructose (MPBio) and $18.9 \mathrm{~g} / \mathrm{L}$ sucrose (VWR). Control mice were fed a standard diet with $11 \mathrm{kcal} \%$ fat with corn starch (D12328i; Research Diets).

\section{Flow cytometry and cell sorting}

Cells were pre-incubated with 2.4G2 antibody (Bioceros) to block Fc receptors and stained with appropriate antibodies at $4{ }^{\circ} \mathrm{C}$ in the dark for 30-45 minutes. Cell viability was assessed using Fixable Viability dyes (eFluor780 or eFluor506; Thermo Fischer) and cell suspensions were analyzed with a BD FACSymphony or purified using a BD Symphony S6, BD FACSAria II or III. Nuclei were sorted on basis of DAPI positivity and size. Analysis was performed with FlowJo software (BD). Intracellular staining for CD207 was performed by fixing and permeabilizing extracellularly stained cells according to the manufacturer's instructions using the FoxP3 Fixation/Permeabilization Kit (Thermo Fischer).

\section{Confocal microscopy}

Confocal staining was performed as described previously (Bonnardel et al., 2019). Immediately after sacrificing mice with $\mathrm{CO}_{2}$, inferior vena cava were cannulated and livers were perfused $(4 \mathrm{~mL} / \mathrm{min}$ ) with Antigenfix (Diapath) for 5 min at room temperature. After excision, 2-3 $\mathrm{mm}$ slices of livers were fixed further by immersion in Antigenfix for $1 \mathrm{~h}$ at $4^{\circ} \mathrm{C}$, washed in PBS, infused overnight in $34 \%$ sucrose and frozen in Tissue-Tek OCT compound (Sakura Finetek). $20 \mu \mathrm{m}$-thick slices cut on a cryostat (Microm HM 560, Thermo Scientific) were rehydrated in PBS for 5 min, permeabilized with 0,5\% saponin and non-specific binding sites were blocked for 30 min with $2 \%$ bovine serum albumin, $1 \%$ fetal calf serum and $1 \%$ donkey or goat serum for 30 minutes. Tissue sections were labeled overnight at $4^{\circ} \mathrm{C}$ with primary antibodies followed by incubation for $1 \mathrm{~h}$ at room temperature with secondary antibodies. When two rat antibodies were used on the same section, the directly conjugated rat antibody was incubated for $1 \mathrm{~h}$ after staining with the unconjugated and anti-rat secondary and after an additional blocking step with $1 \%$ rat serum for 30 minutes. Slides were mounted in ProLong Diamond, imaged with a Zeiss LSM780 confocal microscope (Carl Zeiss, Oberkochen, Germany) with spectral detector and using spectral unmixing and analyzed using ImageJ and QuPath software.

\section{Confocal microscopy combined with RNAScope}

Experiments were performed using the RNAScope Multiplex Fluorescent V2 Assay kit (ACDBio 323100). Probes targeting intronic regions for Hs-Cd5I (ACDBio 850511), Mfa-Cd5I (ACDBio 873211), Mm-Cd5I (ACDBio 573271), Mm-Flt3 (ACDBio 487861), MmXcr1 (ACDBio 562371), Mm-Mafb (ACDBio 438531) and Mm-Mgl2-O1 (ACDBio 822901) were custom-designed and synthesized. They were then labelled with TSA opal 520 (PerkinElmer FP1487001KT), TSA opal 540 (PerkinElmer FP1494001KT), TSA opal 570 (PerkinElmer FP1488001KT), TSA opal 620 (PerkinElmer FP1495001KT) or TSA opal 650 (PerkinElmer FP1496001KT). Tissues were fixed for 16 hours in AntigenFix (Diapath P0016), dehydrated and embedded in OCT as described above. Slices were pretreated with hydrogen peroxide for $10 \mathrm{~min}$ and protease III for $20 \mathrm{~min}$. The recommended Antigen retrieval step was not performed in order to preserve epitope integrity. Probes were hybridized and amplified according to the manufacturer's instructions. Slides were then stained for protein markers as described above.

\section{Visium}

Mice were euthanized by means of carbon dioxide $\left(\mathrm{CO}_{2}\right)$ overdose. The liver was excised and consequently trimmed, on ice, to smaller tissue pieces fitting the 10X Visium capture area. Trimmed tissue pieces were embedded in Tissue-Tek ${ }^{\circledR}$ O.C.T. ${ }^{\mathrm{TM}}$ Compound (Sakura) and snap frozen in isopentane (Sigma) chilled by liquid nitrogen. Embedded tissue pieces where stored at $-80^{\circ} \mathrm{C}$ until cryosectioning.

A 10X Visium Spatial Gene expression slide was placed in the cryostat (Cryostar NX70 Thermo Fisher) 30 minutes prior to cutting. $10 \mu \mathrm{m}$ sections where cut and placed within the capture area. Single 10X Visium Spatial Gene expression slides were stored in an airtight container at $-80^{\circ} \mathrm{C}$ until further processing.

10X Visium cDNA libraries were generated according the manufacturer's instructions. In short: Tissue sections where fixed in chilled Methanol. A H\&E staining was performed to assess tissue morphology and quality. Tissue was lysed and reverse transcription was performed followed by second strand synthesis and cDNA denaturation. cDNA was transferred to a PCR tube and concentration was determined by qPCR. Spatially barcoded, full length cDNA was amplified by PCR. Indexed sequencing libraries where generated via End Repair, A-tailing, adaptor ligation and sample index PCR. Full length cDNA and indexed sequencing libraries were analyzed using the Qubit 4 fluorometer (Thermo Fisher) and Agilent 2100 BioAnalyzer.

\section{Visium highly multiplexed protein}

Liver slices were prepared as described above for the classical Visium protocol. Slices were dried for 1 min at $37^{\circ} \mathrm{C}$ and subsequently fixed using 1\% paraformaldehyde in PBS. Next, slices were blocked for 30 min (2\% BSA, 0.1ug/ul Salmon Sperm, 0.5\% Saponin, 
$1 \mathrm{U} / \mu \mathrm{l}$ protector RNase inhibitor (Roche) in 3X SSC) and incubated with the oligo-conjugated antibody staining mix (2\% BSA, $0.1 \mu \mathrm{g} / \mu \mathrm{l}$ Salmon Sperm, $0.5 \%$ Saponin, $1 \mathrm{U} / \mu$ l protector RNase inhibitor, 10uM polyT-blocking oligo (TTTTTTTTTTTTTTTTT $\mathrm{T}^{\star} \mathrm{T}^{\star} / 3$ InvdT/), in $3 \mathrm{X} \mathrm{SSC}$ ) for $1 \mathrm{~h}$ at $4^{\circ} \mathrm{C}$. Slides were mounted ( $90 \%$ glycerol, $1 \mathrm{U} / \mu$ l protector RNase inhibitor) and imaged on Zeiss Axioscan Z1 at 20X magnification. Samples were then processed for a transcriptomic experiment as per manufacturer's instructions (Visium, 10X Genomics) with modifications to also capture antibody tags. In short, tissue was permeabilized using Tissue Removal Enzyme (Tissue Optimization kit, 10x Genomics) for 9 minutes, as determined by a tissue optimization experiment (10X Genomics, Visium Spatial Tissue Optimization). After reverse transcription, $2 \mu \mathrm{l}$ of $100 \mu \mathrm{M}$ FB additive primer (CCTTGGCACCCGAGAATT ${ }^{\star} \mathrm{C}^{\star}$ $\left.C^{\star} A\right)$ per sample was added to the second strand synthesis mix. During cDNA amplification $1 \mu$ of $0,2 \mu \mathrm{M}$ FB additive primer (CCTTGGCACCCGAGAATT ${ }^{*} \mathrm{C}^{*} \mathrm{C}^{*} \mathrm{~A}$ ) was added. After cDNA amplification, antibody products and mRNA derived cDNA were separated by $0.6 \mathrm{X}$ SPRI select. The purified full-length cDNA fraction was quantified by qRT-PCR using KAPA SYBR FAST-qPCR kit on a PCR amplification and detection instrument. After enzymatic fragmentation indexed sequencing libraries were generated via End Repair, A-Tailing, adaptor ligation and sample index PCR. The supernatant containing antibody product was cleaned up by two rounds of $1.9 \mathrm{X}$ SPRI select. Next, $45 \mu \mathrm{l}$ of the purified antibody fraction was amplified with a 96 deep well reaction module: $95^{\circ} \mathrm{C}$ for $3 \mathrm{~min}$; cycled 8 times: $95^{\circ} \mathrm{C}$ for $20 \mathrm{~s}, 60^{\circ} \mathrm{C}$ for $30 \mathrm{~s}$, and $72^{\circ} \mathrm{C}$ for $20 \mathrm{~s} ; 72^{\circ} \mathrm{C}$ for $5 \mathrm{~min}$; end at $4^{\circ} \mathrm{C}$. ADT libraries were purified once more with 1.6X SPRI select. Full length cDNA, indexed cDNA libraries and antibody libraries were analyzed using the Qubit 4 fluorometer (Thermo Fisher) and Agilent 2100 Bioanalyzer. The separation of the cDNA and ADT libraries were performed according to the manufacturer's instructions (10X genomics).

\section{MICS (MACSima ${ }^{\top}$ Imaging Cyclic Staining) technology on the MACSima ${ }^{\top}$ Imaging System by Miltenyi Biotec B.V. \&}

\section{Co. KG}

The MACSima ${ }^{\mathrm{TM}}$ Imaging System is a fully automated instrument combining liquid handling with widefield microscopy for cyclic immunofluorescence imaging. In brief, staining cycles consisted of the following automated steps: immunofluorescent staining, sample washing, multi-field imaging, and signal erasure (photobleaching or REAlease).

Cryosectioned slices on slides were taken out of the $-80^{\circ} \mathrm{C}$ storage and the appropriate MACSWell ${ }^{\mathrm{TM}}$ imaging frame was mounted immediately on the slide. An appropriate volume of ice-cold 4\% PFA solution was added (according to the MACSWell ${ }^{\mathrm{TM}}$ imaging frames datasheet) and incubated for 10 minutes at room temperature. The slide was washed three times with MACSima Running Buffer. After washing the appropriate initial sample volume of MACSima Running Buffer was added (according to the MACSWell ${ }^{\mathrm{TM}}$ imaging frames datasheet). Right before the start of the MACSima ${ }^{{ }^{T M}}$ instrument a DAPI pre-staining was performed: the MACSima Running Buffer was removed from the sample to be analysed and stained for 10 min with a 1:10 dilution of a DAPI staining solution (volume depends on working volume for the different MACSwell ${ }^{\mathrm{TM}}$ formats, see datasheet). The DAPI staining solution was removed and 3 washing steps were performed (MACSima Running Buffer). Finally, the initial sample volume of MACSima Running Buffer was added. Details of the antibodies used can be found in the key resources table.

\section{Molecular cartography ${ }^{\mathrm{TM}}$ \\ Tissue sections}

Liver was frozen and sectioned as described above for Visium analysis and liver slices were placed within capture areas on Resolve BioScience slides. Samples were then sent to Resolve BioSciences on dry ice for analysis. Upon arrival, tissue sections were thawed and fixed with 4\% v/v Formaldehyde (Sigma-Aldrich F8775) in 1x PBS for 30 min at $4{ }^{\circ} \mathrm{C}$. After fixation, sections were washed twice in 1x PBS for two min, followed by one min washes in 50\% Ethanol and $70 \%$ Ethanol at room temperature. Fixed samples were used for Molecular Cartography ${ }^{\mathrm{TM}}$ (100-plex combinatorial single molecule fluorescence in-situ hybridization) according to the manufacturer's instructions (protocol 3.0; available for download from Resolve's website to registered users), starting with the aspiration of ethanol and the addition of buffer BST1 (step 6 and 7 of the tissue priming protocol). Briefly, tissues were primed followed by overnight hybridization of all probes specific for the target genes (see below for probe design details and target list). Samples were washed the next day to remove excess probes and fluorescently tagged in a two-step color development process. Regions of interest were imaged as described below and fluorescent signals removed during decolorization. Color development, imaging and decolorization were repeated for multiple cycles to build a unique combinatorial code for every target gene that was derived from raw images as described below.

\section{Probe Design}

The probes for 100 genes were designed using Resolve's proprietary design algorithm. Briefly, the probe-design was performed at the gene-level. For every targeted gene all full-length protein-coding transcript sequences from the ENSEMBL database were used as design targets if the isoform had the GENCODE annotation tag 'basic' (Frankish et al., 2019; Yates et al., 2020). To speed up the process, the calculation of computationally expensive parts, especially the off-target searches, the selection of probe sequences was not performed randomly, but limited to sequences with high success rates. To filter highly repetitive regions, the abundance of $k$-mers was obtained from the background transcriptome using Jellyfish (Marçais and Kingsford, 2011). Every target sequence was scanned once for all $k$-mers, and those regions with rare $k$-mers were preferred as seeds for full probe design. A probe candidate was generated by extending a seed sequence until a certain target stability was reached. A set of simple rules was applied to discard sequences that were found experimentally to cause problems.

After these fast screens, every kept probe candidate was mapped to the background transcriptome using ThermonucleotideBLAST (Gans and Wolinsky, 2008) and probes with stable off-target hits were discarded. Specific probes were then scored based 
on the number of on-target matches (isoforms), which were weighted by their associated APPRIS level (Rodriguez et al., 2018), favoring principal isoforms over others. A bonus was added if the binding-site was inside the protein-coding region. From the pool of accepted probes, the final set was composed by greedily picking the highest scoring probes. Probe details are included in the key resources table.

Imaging

Samples were imaged on a Zeiss Celldiscoverer 7, using the 50x Plan Apochromat water immersion objective with an NA of 1.2 and the $0.5 x$ magnification changer, resulting in a 25x final magnification. Standard CD7 LED excitation light source, filters, and dichroic mirrors were used together with customized emission filters optimized for detecting specific signals. Excitation time per image was $1000 \mathrm{~ms}$ for each channel (DAPI was $20 \mathrm{~ms}$ ). A z-stack was taken at each region with a distance per z-slice according to the NyquistShannon sampling theorem. The custom CD7 CMOS camera (Zeiss Axiocam Mono 712, $3.45 \mu \mathrm{m}$ pixel size) was used.

For each region, a z-stack per fluorescent color (two colors) was imaged per imaging round. A total of 8 imaging rounds were done for each position, resulting in $16 z$-stacks per region. The completely automated imaging process per round (including water immersion generation and precise relocation of regions to image in all three dimensions) was realized by a custom python script using the scripting API of the Zeiss ZEN software (Open application development).

Spot segmentation

The algorithms for spot segmentation were written in Java and are based on the ImageJ library functionalities. Only the iterative closest point algorithm is written in $\mathrm{C}++$ based on the libpointmatcher library (https://github.com/ethz-asl/libpointmatcher).

Preprocessing

As a first step all images were corrected for background fluorescence. A target value for the allowed number of maxima was determined based upon the area of the slice in $\mu \mathrm{m}^{2}$ multiplied by the factor 0.5 . This factor was empirically optimized. The brightest maxima per plane were determined, based upon an empirically optimized threshold. The number and location of the respective maxima was stored. This procedure was done for every image slice independently. Maxima that did not have a neighboring maximum in an adjacent slice (called z-group) were excluded. The resulting maxima list was further filtered in an iterative loop by adjusting the allowed thresholds for (Babs-Bback) and (Bperi-Bback) to reach a feature target value (Babs: absolute brightness, Bback: local background, Bperi: background of periphery within 1 pixel). This feature target values were based upon the volume of the 3D-image. Only maxima still in a z-group of at least 2 after filtering were passing the filter step. Each z-group was counted as one hit. The members of the z-groups with the highest absolute brightness were used as features and written to a file. They resemble a 3D-point cloud.

Final signal segmentation and decoding

To align the raw data images from different imaging rounds, images had to be corrected. To do so the extracted feature point clouds were used to find the transformation matrices. For this purpose, an iterative closest point cloud algorithm was used to minimize the error between two point-clouds. The point clouds of each round were aligned to the point cloud of round one (reference point cloud). The corresponding point clouds were stored for downstream processes. Based upon the transformation matrices the corresponding images were processed by a rigid transformation using trilinear interpolation.

The aligned images were used to create a profile for each pixel consisting of 16 values (16 images from two color channels in 8 imaging rounds). The pixel profiles were filtered for variance from zero normalized by total brightness of all pixels in the profile. Matched pixel profiles with the highest score were assigned as an ID to the pixel.

Pixels with neighbors having the same ID were grouped. The pixel groups were filtered by group size, number of direct adjacent pixels in group, number of dimensions with size of two pixels. The local 3D-maxima of the groups were determined as potential final transcript locations. Maxima were filtered by number of maxima in the raw data images where a maximum was expected. Remaining maxima were further evaluated by the fit to the corresponding code. The remaining maxima were written to the results file and considered to resemble transcripts of the corresponding gene. The ratio of signals matching to codes used in the experiment and signals matching to codes not used in the experiment were used as estimation for specificity (false positives).

Downstream analysis

Final image analysis was performed in ImageJ using genexyz Polylux tool plugin from Resolve BioSciences to examine specific Molecular Cartography ${ }^{\mathrm{TM}}$ signals.

RNA sequencing, CITE-seq, and qPCR

Sorting and RNA Isolation

40000-160000 cells of interest from livers of the different species were FACS-purified and pelleted by centrifugation at $400 \mathrm{~g}$ for 5 mins. To ensure sufficient numbers of all cell types were present in our analyses, depending on the sample distinct populations of cells including Live CD45 ${ }^{+}$, Live CD45- Hepatocytes, Myeloid cells and Stromal cells were FACS-purified. When CITE-seq was to be performed, cells were then stained with $2.4 \mathrm{G} 2$ antibody to block Fc receptors and CITE-seq antibodies for $20 \mathrm{mins}$ at $4{ }^{\circ} \mathrm{C}$, before being washed in excess PBS with $2 \%$ FCS and 2mM EDTA. Antibody details are included in the key resources table. 40000-100000 nuclei were also FACS-purified based on DAPI expression. These were sorted into BSA coated tubes and pelleted by centrifuging for 3 mins at $400 \mathrm{~g}$ and 5 mins at $600 \mathrm{~g}$ sequentially. Cells/Nuclei were then resuspended in PBS with $0.04 \% B S A$ at $\sim 1000$ cells $/ \mathrm{ml}$. Cell suspensions (target recovery of 8000-10000 cells) were loaded on a GemCode Single-Cell Instrument (10x Genomics, Pleasanton, CA, USA) to generate single-cell Gel Bead-in-Emulsions (GEMs). Single-cell RNA-Seq libraries were prepared using GemCode Single-Cell 3'Gel Bead and Library Kit (10x Genomics, V2 and V3 technology) according to the manufacturer's 
instructions. Briefly, GEM-RT was performed in a 96-Deep Well Reaction Module: $55^{\circ} \mathrm{C}$ for $45 \mathrm{~min}, 85^{\circ} \mathrm{C}$ for 5 min; end at $4^{\circ} \mathrm{C}$. After RT, GEMs were broken down and the cDNA was cleaned up with DynaBeads MyOne Silane Beads (Thermo Fisher Scientific, 37002D) and SPRIselect Reagent Kit (SPRI; Beckman Coulter; B23318). cDNA was amplified with 96-Deep Well Reaction Module: $98^{\circ} \mathrm{C}$ for $3 \mathrm{~min}$; cycled 12 times : $98^{\circ} \mathrm{C}$ for $15 \mathrm{~s}, 67^{\circ} \mathrm{C}$ for $20 \mathrm{~s}$, and $72^{\circ} \mathrm{C}$ for $1 \mathrm{~min} ; 72^{\circ} \mathrm{C}$ for $1 \mathrm{~min}$; end at $4^{\circ} \mathrm{C}$. Amplified cDNA product was cleaned up with SPRIselect Reagent Kit prior to enzymatic fragmentation. Indexed sequencing libraries were generated using the reagents in the GemCode Single-Cell 3' Library Kit with the following intermediates: (1) end repair; (2) A-tailing; (3) adapter ligation; (4) post-ligation SPRlselect cleanup and (5) sample index PCR. Pre-fragmentation and post-sample index PCR samples were analyzed using the Agilent 2100 Bioanalyzer.

qPCR

RNA was extracted from 10000 sorted cells (gated using strategies shown) from livers of C57BL/6 mice using a RNeasy Plus micro kit (QIAGEN). Sensifast cDNA synthesis kit (Bioline) was used to transcribe total RNA to cDNA. Real-time RT-PCR using SensiFast SYBR No-Rox kit (Bioline) was performed to determine gene expression, therefore a PCR amplification and detection instrument LightCycler 480 (Roche) was used. Gene expression was normalized to $\beta$-actin gene expression. Primers used in the study can be found in the key resources table.

RNA sequencing analysis

sc/snRNA-seq libraries were loaded on an Illumina HiSeq or Illumina NovaSeq 6000 with sequencing settings recommended by $10 \mathrm{X}$ Genomics (26/8/0/98 - 2.1pM loading concentration, ADT and cDNA libraries were pooled in a 25:75 ratio). Visium sequencing libraries were loaded on an Illumina NovaSeq 6000 with sequencing settings recommended by 10X Genomics (28/10/10/75 $2.1 \mathrm{pM}$ loading concentration). Sequencing was performed at the VIB Nucleomics Core (VIB, Leuven). The demultiplexing of the raw data was performed using CellRanger software (10x - version 3.1.0; cellranger mkfastq which wraps Illumina's bcl2fastq). The reads obtained from the demultiplexing were used as the input for 'cellranger count' (CellRanger software), which aligns the reads to the mouse reference genome $(\mathrm{mm} 10)$ using STAR and collapses to unique molecular identifier (UMI) counts. The result is a large digital expression matrix with cell barcodes as rows and gene identities as columns.

Preprocessing data

To remove ambient RNA, the FastCAR R package (v0.1.0) with a contamination chance cutoff of 0.05 was run on the samples separately before merging them. The UMI cut off was determined individually for the different samples, using the CellRanger web_summary output plot (see GitHub). The Scater R package (v1.14.6) was used for the preprocessing of the data. The workflow to identify the outliers, based on 3 metrics (library size, number of expressed genes and mitochondrial proportion) described by the Marioni lab (Lun et al., 2016) was followed. As a first step cells with a value $x$ median absolute deviation (MADs) higher or lower than the median value for each metric were removed. This value was determined individually for the different datasets (see github). Secondly, the runPCA function (default parameters) of the Scater R package was used to generate a principal component analysis (PCA) plot. The outliers in this PCA plot were identified by the R package mvoutlier. By creating the Seurat object, genes that didn't have an expression in at least 3 cells were removed. To normalize, scale and detecting the highly variable genes, the R package SCTransform (v0.2.1) was used. If batch correction (on sample level) was needed, the NormalizeData (log2 transformation), FindVariableFeatures and ScaleData functions of the Seurat R package (v3.1.2) were used in combination with the Harmony R package (v1.0). The Seurat pipeline was followed to find the clusters and create the UMAP plots. The number of principal components used for the clustering and the resolution were determined individually for the different datasets (see GitHub). On these initial UMAP plots we did multiple rounds of cleaning by removing proliferating and contaminating (e.g. doublets) cells. For non CITE-seq datasets the count data for the clean cells acquired by the previous steps were further processed with the scVI model (scvi Python package v0.6.7) (Lopez et al., 2018). Datasets including Cite-seq samples were further processed with the TotalVI model (Gayoso et al., 2021). The workflows described on scvi-tools.org were followed to generate new UMAPs, DEGs and DEPs. This information was further processed with the pheatmap R package (v1.0.12) to create heatmaps using the normalized values (denoised genes) calculated in the scVI/TotalVI workflow. The plots showing the expression of certain genes or proteins are created with the ggplot2 R package (v3.2.1) with a quantile cut off of 0.01 .

For mouse all the ABs from the whitelist (181 ABs) were loaded into TotalVI, while for the other species only the added ABs were loaded into TotalVI. For the 'human liver-pool of techniques and patients' we noticed that the batch correction (between samples) faced difficulties for the hepatocytes and stellate cells as the cells all originated from snRNA-Seq samples, while the other cell types originated from both snRNA-seq and scRNA-seq samples. To overcome this issue we randomly allocated $30 \%$ of the hepatocytes to scRNA-seq samples which were not CITE-seq samples. We did the same for $30 \%$ of the stellate cells.

Heatmaps were made by scaling the normalized values (denoised values; calculated in the scVl/TotalVI workflow) using the scale_quantile function of the SCORPIUS R package (v1.0.7) and the pheatmap R package (v1.0.12). The plots showing the expression of certain genes or proteins were created based on the normalized values (denoised values) using a quantile cutoff of 0.99 and via either the ggplot2 R package (v3.2.1) or the scanpy.pl.umap function of the Scanpy Python package (v1.5.1).

Conserved human-mouse KC signature

To find the conserved human and mouse $\mathrm{KC}$ markers we started by identifying the human KC markers. We mapped the annotation of the human myeloid UMAP on the human pool of techniques/patients UMAP to identify the real KCs in this last UMAP. The real KCs were identified as the top part of the mac cluster. Using this new annotation we then calculated the DE genes and DE proteins for each cluster. Some genes are listed as marker for multiple clusters, only for the cluster where the gene had the highest score (raw_normalized_mean1/raw_normalized_mean2*Ifc_mean), the gene was kept as marker. This way we found 110 potential human KC 
markers. We then created a heatmap of these 110 genes (using denoised gene values scaled between 0 and 1 ) and filtered this heatmap by removing the genes where the scaled normalized value was higher than 0.50 in more than $30 \%$ of the cells of a certain cell type other than KCs. Except for the macs, we only removed a gene when it had a scaled normalized value higher than 0.50 in more than $70 \%$ of the macs. After this filtering we ended up with 36 human KC markers. Next we converted these human gene symbols into MGI IDs via the BioMart tool on the HGNC website (https://biomart.genenames.org/martform/\#!/default/HGNC?datasets=hgnc_ gene_mart). We found a MGI ID for 30 genes. We then converted these MGI IDs into mouse gene symbols via the MGI webtool (http://www.informatics.jax.org/batch/).

To identify the mouse KC markers we similarly mapped the annotation of the mouse myeloid UMAP on the mouse pool of techniques UMAP to identify the real KCs in this last UMAP. The real KCs matched with the mac cluster. Similarly as in human, the DE genes for each cluster was calculated and genes listed as marker for multiple clusters were dealt with in a similar way. This way we found 264 potential mouse KC markers. We then removed the genes that had a score (raw_normalized_mean1/ raw_normalized_mean2*Ifc_mean) lower than 10 and ended up with 214 genes. We then created a heatmap of these 214 genes (using denoised gene values scaled between 0 and 1) and filtered this heatmap by removing the genes where the scaled normalized value was higher than 0.50 in more than $30 \%$ of the cells of a certain cell type other than KCs. After this filtering we ended up with 68 mouse KC markers. Next we converted these mouse gene symbols into MGI IDs via the MGI webtool (http://www.informatics.jax.org/batch/). We then converted these MGI IDs into human gene symbols via the BioMart tool on the HGNC website (https://biomart.genenames.org/martform/\#!/default/HGNC?datasets=hgnc_gene_mart) and ended up with 60 genes.

At this point we found 30 human $\mathrm{KC}$ markers and 60 mouse $\mathrm{KC}$ markers. In a next step, we only kept the human $\mathrm{KC}$ markers that we identified as a Highly Variable Gene (HVG) in the mouse pool of techniques UMAP (20 genes) and the mouse KC markers that were identified as HVGs in the human pool of the techniques UMAP (30 genes). We next put these 20 mouse KC markers in SingleCellSignatureExplorer (Pont et al., 2019) to see where these genes are enriched in the mouse pool of techniques UMAP. In order to only get an enrichment in the KCs we decided to only use top 10 mouse KC markers (ordered on score), together with S/c40a1 and Hmox1. We then started to add the top human KC markers as long as we keep the enrichment solely in the KCs. This way we ended up with final list of 15 human-mouse conserved $\mathrm{KC}$ markers.

We next converted these KC markers into the monkey, pig, chicken or zebrafish orthologs by looking up the human gene symbol on NCBI (https://www.ncbi.nlm.nih.gov/search/) and checking if there is an ortholog of the species of interest listed under the 'Ortholog' tab. The found orthologs were then used as input for the SingleCellSignatureExplorer tool.

Conversion of the CITE-seq data into a flow cytometry file

The protein normalized values (denoised values; calculated in the TotalVI workflow) were converted into an FCS file using the write.FCS function of the flowCore R package (v1.50.0).

\section{Preprocessing Visium data}

We first removed per sample all spots that were clear outliers compared to the location of the tissue. Each sample was then normalized individually using the SCTransform function of the Seurat R package (v3.2.3) with default parameters. All samples were then merged with the merge function of the Seurat R package (v3.2.3) with default parameters. Next, we determined the HVGs, created a PCA plot, performed clustering and created an UMAP plot as described in the spatial workflow available on the Seurat website (https://satijalab.org/seurat/articles/spatial_vignette.html). Clusters which showed high mitochondrial gene expression were removed. Spots located at the darker parts of the tissue were also removed as these parts are considered to be dead tissue or of bad quality.

\section{Modelling of Visium data}

\section{Probabilistic graphical modeling}

For modelling the cell type composition and zonation, spatial CITE-seq and transcriptomics data were analyzed using probabilistic graphical models, similar to what is used in tools such as cell2location and scVI. In brief, transcriptomics data was modelled as a NegativeBinomial distribution, parameterized with a mean $\mu$ and dispersion $\theta$, the latter optimized as a free parameter for each gene. Visium Highly Multiplexed Protein data was modelled as a mixture of NegativeBinomials, with a $\mu_{\text {background }}$ and $\mu_{\text {foreground }}$ and a shared dispersion $\theta$. The actual foreground/background signal within a modality was modelled as a $\rho$ that depends on the latent space, and which is multiplied with the empirical library size to get $\mu$. For Visium Highly Multiplexed Protein, $\rho_{\text {background }}$ was modelled as a latent variable specific for each gene. $\rho_{\text {foreground }}$ for Visium Highly Multiplexed Protein and $\rho$ for RNA-seq were modelled as deterministic functions depending on the use case as described in the following paragraphs. The posterior of the probabilistic graphical model was inferred using black-box variational inference (Ranganath et al., 2013), in which the variational distribution was specified as a diagonal Normal distribution, transformed into the correct domain using transforms $\left(\mathbb{R} \rightarrow \mathbb{R}^{+}: e^{x}, \mathbb{R} \rightarrow \Delta: \frac{e^{x_{i}}}{\sum_{i} e^{x_{i}}}, \mathbb{R} \rightarrow\right.$ $\left.[0,1]: \frac{e^{x}}{e^{x}+1}\right)$. Free parameters within this model were optimized using gradient descent, with the ELBO as loss function and Adam as optimizer as implemented in Pytorch (Paszke et al., 2019) (pytorch.org). We used a learning rate of 0.01 for variational parameters, and 0.001 for parameters of the amortization functions. 


\section{Reference for deconvolution}

To calculate the average expression of each gene within a cell type, we used a linear model in which both $\rho$ and $\theta$ were modelled as a latent variable specific for each gene and cell type. The $\rho$ for nuclei were multiplied with a gene-specific correction factor (optimized as a latent variable) that corrected for differences between scRNA-seq and snRNA-seq. Given that spatial transcriptomics data sequences the whole cell, the uncorrected $\rho$ values were used for spatial deconvolution.

\section{Deconvolution}

To infer the proportions of each cell type within a spot, we used a model in which the gene expression is modelled as a linear combination of cell type proportions and average expression in each cell type:

$\rho_{\text {spot,gene }}=\nu_{\text {spot,celltype }} \times \rho_{\text {celltype,gene }}$

For $\rho_{\text {celltype.gene }}$ we adapted the values from the reference, but included

- A capture bias per gene, which corrects for technical and biological differences between spatial and sc/sn-RNA-seq. The capture bias was modelled as a latent variable with prior $\operatorname{Normal}(0,1)$

- A red blood cell cell type, which was not included in the reference dataset but nonetheless had a dominant presence in the spatial data. The $\rho$ of this cell type was set to zero for all genes except $H b b-b t, H b b-b s, H b a-a 1, H b a-a 2$ for mouse and $H B B, H B A 1, H B A 2$ for human, which were modelled as free parameters.

- Similarly, the expression of complement factors $(C 3, C 2, C 4 B / C 4 b)$ within hepatocytes was modelled as free parameters.

A background signal shared for all spots was also modelled as follows:

$\mu_{\text {spot,gene }}=\rho_{\text {spot,gene }} \times$ lib $\times$ foreground $+\mu_{\text {background }}$

With foreground $\in[0,1]$ a latent variable specific to each spot and $\mu_{\text {background }} \in \mathbb{R}$ a latent variable specific to each gene.

A likelihood ratio test was used to assess whether a cell type was significantly present in a spot. Specifically, if $x$ is the gene expression of all genes at a particular spot, we used Monte Carlo samples from the posterior to estimate:

$$
\frac{P\left(x \mid \nu_{\text {celltype }}\right)}{P\left(x \mid \nu_{\text {celltype }}=0\right)}
$$

A cell type was deemed significantly present if the log-likelihood was higher than 10.

\section{Zonation}

The zonation of spots was modelled as a univariate latent variable $z \sim$ Uniform $(0,1)$ specific to each spot. This latent variable influenced the gene expression $\rho$ using a spline function by using a gaussian basis function $(\sigma=0.05)$ with 10 knots at uniform fixed positions. The coefficients of this spline were modelled as a latent variable specific for each gene, with prior a Gaussian random walk distribution, and the step $\sim \operatorname{Normal}\left(0, \sigma_{\text {gene }}\right) . \sigma_{\text {gene }}$ was determined empirically as 2 times the standard deviation of the log1p transformed expression values in the whole dataset. The variational parameters of the zonation $\mu_{z}$ and $\sigma_{z}$ were not optimized directly but were estimated using an amortization function. This amortization function used the count matrix as input, and estimated the variational parameters using the following layers: Linear (with 100 output dimensions), BatchNorm, ReLU, Linear (again with 100 output dimensions), ReLU, and a final Linear layer. This amortization function was used to transfer the zonation onto a different dataset, i.e., 1) to transfer the zonation trained on mouse spatial transcriptomics onto mouse Visium highly multiplexed protein and 2) to transfer the zonation trained on human low steatosis $(<10 \%)$ onto human high steatosis $(>30 \%)$.

\section{Differential abundance along zonation}

To determine the differential abundance of a cell type across zonation, the significant presence of a cell type within a spot $\in\{0,1\}$ was modelled using a spline function with the zonation of a cell type as input. The coefficients of this spline function were modelled as a latent variable with the step size $\sim \operatorname{Normal}(0,1)$. To determine differences in abundance between patients with high and low steatosis, we first modelled the zonation on human data on patients with steatosis $<10 \%$. Potential interaction effects between zonation and steatosis status were then modelled using a spline function as before, but with a separate set of coefficients for both high and low steatosis. A likelihood ratio test was then used to determine whether this interaction was present significantly, by comparing the likelihood of this model with a model with shared coefficients.

\section{Differential NicheNet}

To analyze cell-cell communication in the hepatic mac niches, we applied Differential NicheNet, which is an extension of the default NicheNet pipeline to compare cell-cell interactions between different niches and better predict niche-specific ligand-receptor (L-R) pairs. It uses a flexible prioritization scheme that allows ranking $L-R$ pairs according to several properties, such as niche- and regionspecific expression of the L-R pair, ligand activity, and level of database curation. This in contrast to the default NicheNet pipeline which prioritizes expressed L-R pairs solely based on ligand activity predictions. All analyses were conducted according to the Differential NicheNet tutorial (https://github.com/saeyslab/nichenetr/blob/master/vignettes/differential_nichenet.md). As input to the Differential NicheNet pipeline, we used the data after normalization via SCTransform and integration of scRNA-seq and snRNAseq according to the Seurat procedure for integration (Stuart et al., 2019). 
For the mouse analyses, Differential NicheNet was first performed for the following 3 niche comparisons: 1) KCs versus central vein macs; 2) KCs versus capsule macs; 3) KCs versus LAMs. Following sender cell types were considered for these niches: KC niche: periportal hepatocytes, periportal LSECs, and periportal stellate cells; Central vein mac niche: central vein ECs and central vein fibroblasts; Capsule mac niche: mesothelial cells and capsule fibroblasts; LAM niche: cholangiocytes and bile duct fibroblasts.

Because of the preferentially periportal localization of KCs in the mouse liver, we also included a 'region specificity' factor in the Differential NicheNet prioritization framework. This was done to increase the ranking of ligands that are more strongly expressed in periportal than pericentral niche cells. Periportal sender cells were determined after subclustering based on the following markers: $\mathrm{Hal}$ and Sds for hepatocytes; Mecom, Msr1, and Efnb2 for LSECs; Ngfr, Igfbp3, and Dach1 for stellate cells.

In the heatmap (Figure S8G), we show the prioritization scores of the top 40 ligands (and their highest scoring receptor) in the KC niche (score averaged over the 3 analyses), and of all the non-KC niche L-R pairs with a prioritization score $\geq$ the score of the lowest scoring KC L-R pair of this top 40. For each L-R pair/niche combination, we only displayed the score of the sender cell with the highest score (e.g. for the Csf1-Csf1r interaction in the KC niche, the score is shown for the LSEC-KC interaction because that score was higher than for Stellate-KC and Hepatocyte-KC; in the LAM niche, the score of Csf1-Csf1r is shown for the bile duct fibroblast LAM interaction and not for the cholangiocyte-LAM interaction, etc.).

Because of the strong concordance between the top-ranked L-R pairs in these 3 non-KC mac niches, it was decided to also conduct a subsequent analysis in which the $\mathrm{KC}$ niche is compared against all non-KC hepatic mac niches combined. For this final 'KC versus all non-KC mac analysis', KCs were compared to central vein macs, capsule macs, and LAMs together, with the same sender cell types as described here above (but now analyzed together).

For the human analyses, Differential NicheNet was performed to compare the KC niche with the non-KC mac niches (similarly as the final analysis in mouse). For the KC niche, all hepatocytes, LSECs, and stellate cells were selected as sender cells; and KCs as receiver cells. For the non-KC mac niche, cholangiocytes, fibroblasts, and central vein ECs were considered as the sender cells; Mat. LAMs, Imm. LAMs, and Mac1s as the receiver cells (Figure 4H).

To find KC-niche-specific L-R pairs that are conserved across mouse and human, the individual mouse and human prioritization scores were averaged to form a 'conservation score'. The 40 ligands (and maximally 3 of their highest scoring receptors) with the highest conservation score were selected for further analysis (note: the L-R pair should be expressed by the same sender-receiver pair in both species). In the circos plot (Figure 6C; Gu et al., 2014), only a subset of these top L-R pairs is shown to keep the figure clearly interpretable. Following ligands were not shown: ITGA9, SEMA6D, JAM3, ITGB1 (stellate cells); ITGA9, F8, CD274, HSP90B1 (LSECs); C5, F9, F2, FGA, TF, TTR, COL18A1, COL5A3, SERPINA1, SERPINC1 (hepatocytes). The depicted target genes are KC-specific in both mouse and human, and a top-predicted target according to the NicheNet ligandtarget regulatory potential scores. $\mathrm{NR} 1 \mathrm{H} 3$ was manually added as a NOTCH2 target based on recent studies (Bonnardel et al., 2019).

\section{Staining of human liver paraffin sections}

Resected human liver was fixed in $4 \%$ formalin for $24-48 \mathrm{~h}$ and subsequently embedded in paraffin. Samples were stored for $10-15$ years at RT before analysis. Sections of $6 \mu \mathrm{m}$ thick were cut using a Microm HM360 and mounted on a polarized glass slide. These sections were deparaffinized in xylene and rehydrated in a graded ethanol series. Antigen retrieval was performed by immersing the samples for $5 \mathrm{~min}$ in $\mathrm{pH} 8.3$ TRIS-EDTA at $98^{\circ} \mathrm{C}$. Slides were then cooled to RT and washed in PBS. Confocal staining was performed as described above.

Isolation and culture of BM monocytes with acetylated LDL

BM was isolated from the tibia and femur of mice by centrifugation. Red blood cells were lysed and single cell suspensions were stained with antibodies for flow cytometry. BM monocytes were sorted as live CD45+ CD11b+ Ly6G- Ly6C+ CD115+ cells using a BD FACSAria III. Monocytes were resuspended in DMEM/F12 media supplemented with 10\% FCS, 30ng/ml CSF1, 2mM Glutamine and $100 \mathrm{U} / \mathrm{ml}$ penicillin and streptomycin. 150,000 monocytes were seeded in each well of an adherent 24-well plate pre-coated with bovine collagen type I and cultured overnight (37C, $5 \%$ CO2). The following day 0, 25 or 50ng/ml of ac-LDL was added. Ac-LDL was kindly provided by Sophie Janssens, Ghent, Belgium who received the material from Wilfried Le Goff, Paris, France. 14 hours later cells were harvested and live F4/80+ cells were FACS-purified in RLT plus buffer containing $1 \% \beta$-mercaptoethanol. RNA isolation, cDNA synthesis and qPCR were performed as described above.

\section{Generation of bone marrow chimeras}

Bone marrow chimeras were generated as described previously (Scott et al., 2016). Briefly, 6-12 week old Clec4f-Dtr mice (CD45.1) were anaesthetized by intraperitoneal administration of Ketamine $(150 \mathrm{mg} / \mathrm{kg})$ and Xylazine (10 mg/kg). Mice were lethally irradiated with $8 \mathrm{~Gy}$, with the livers being protected with a 3-cm-thick lead cover. Once recovered from the anesthesia, mice were reconstituted by intravenous administration of $5-10 \times 10^{6}$ BM cells from CD 45.2 Acvrl $1^{f / / f l}$ or Fcgr1-CrexAcvrl ${ }^{f / / f} /$ mice. 4 weeks after reconstitution mice were administrated a single dose of 500ng DT via intraperitoneal injection to deplete KCs. Chimerism was assessed 7 or 13 days later by flow cytometry and compared with chimerism levels in blood Ly6C ${ }^{\text {hi }}$ monocytes. 
Administration of Fc traps

Clec4f-Dtr mice were administered 10mg/kg ALK1Fc, TGF $\beta$ RIIFc or appropriate isotype controls (hlgG1 and mlgG2a; Acceleron Pharma) by intraperitoneal injection on days $-1,2,3$ and 5. On Day 0 mice were also administered a single dose of 500ng DT i.p. to deplete KCs. Livers were harvested at day 7 to assess $\mathrm{KC}$ development.

\section{QUANTIFICATION AND STATISTICAL ANALYSIS}

In all experiments, data are presented as mean \pm SEM and/or individual data points are presented unless stated otherwise. Statistical tests were selected based on appropriate assumptions with respect to data distribution and variance characteristics. Details of the precise test used for each analysis can be found in the figure legends. Statistical significance was defined as $p<0.05$. Sample sizes were chosen according to standard guidelines. Number of animals/patients is indicated as " $n$ ". The investigators were not blinded to the group allocation, unless otherwise stated.

\section{ADDITIONAL RESOURCES}

The sc/snRNA-sequencing, CITE-seq FCS files and spatial transcriptomics datasets will be made available for visualization, analysis and download at www.livercellatlas.org. 


\section{Supplementall figures}

A

A ExVivo Digest + scRNA-seg 4
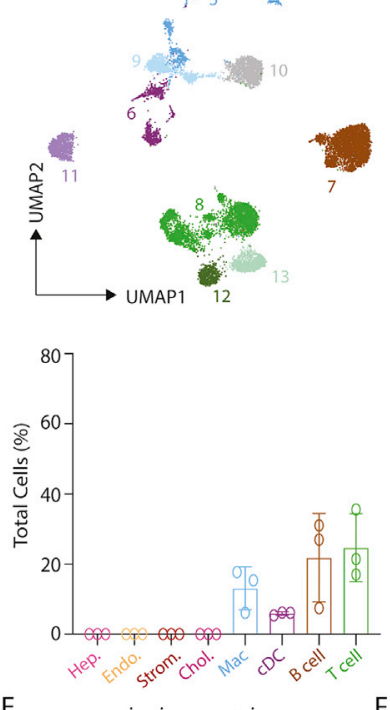

E

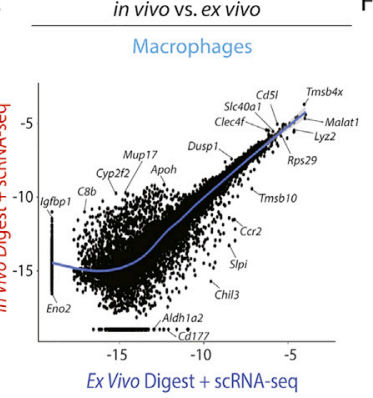

G
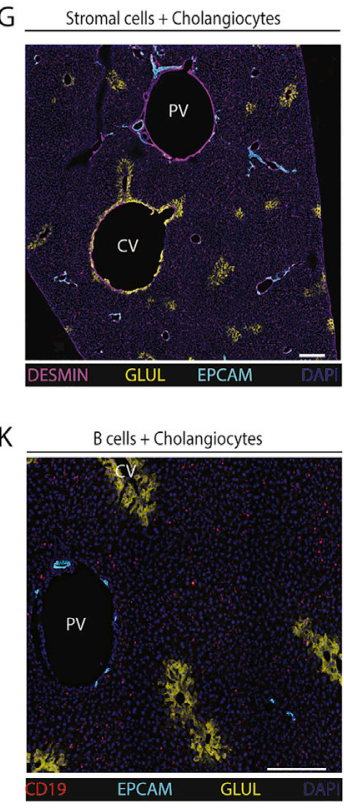

B
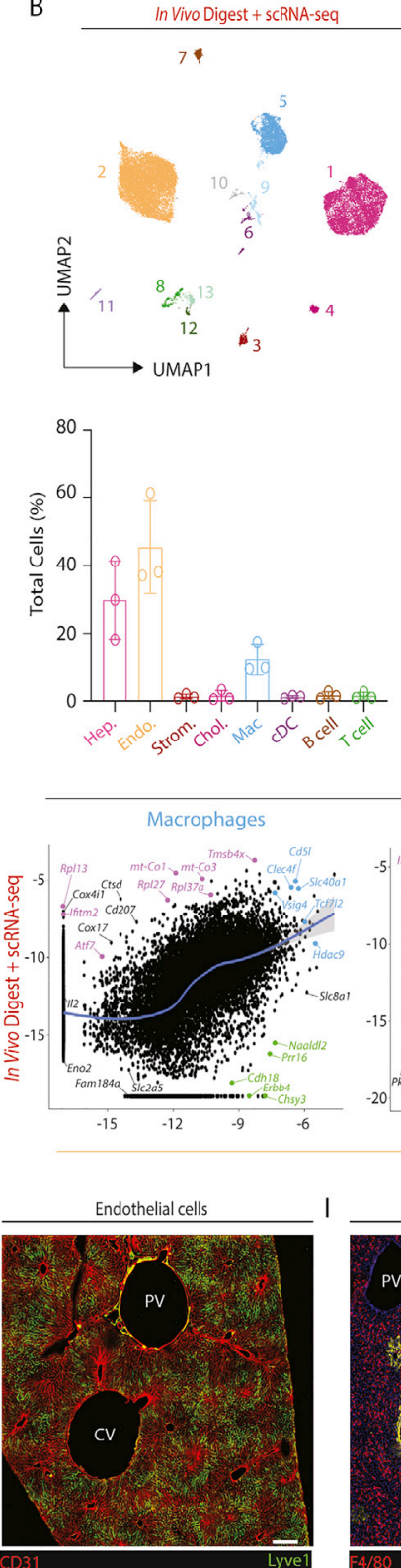

T cells + Cholangiocytes

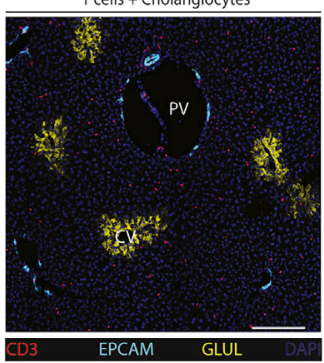

$c$
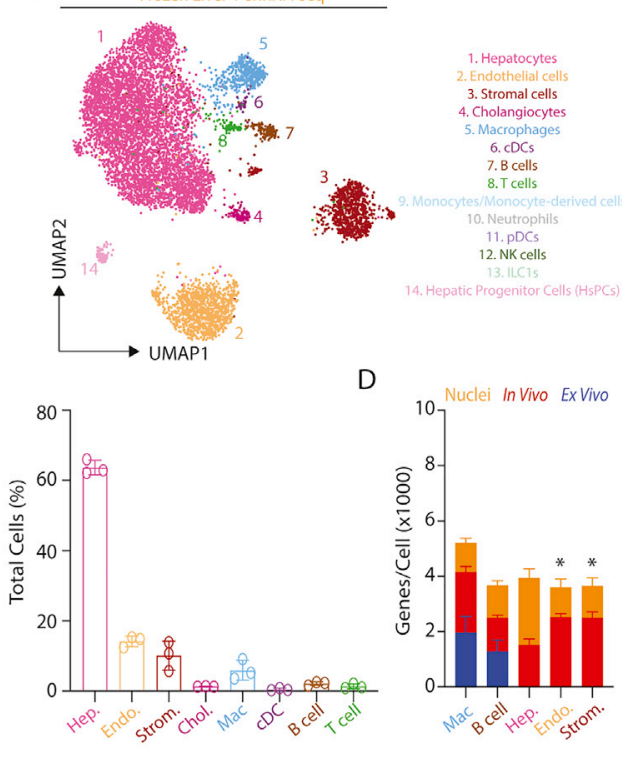
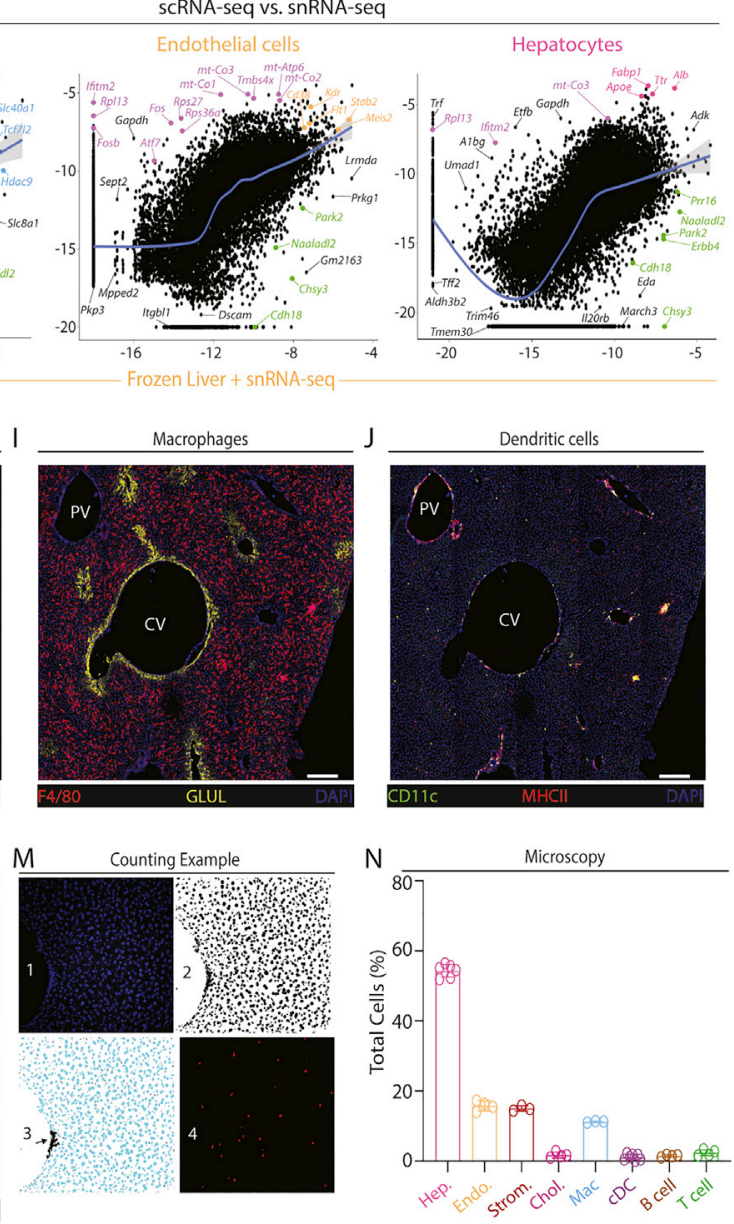

(legend on next page) 
Figure S1. Cell types identified in transcriptomic studies depend upon cell/nuclei isolation technique used, related to Figure 1 Cells were isolated from livers of healthy C57B/l6 mice by either ex vivo or in vivo enzymatic digestion. Alternatively, livers were snap frozen and nuclei subsequently isolated following tissue homogenization by a sucrose gradient ( 3 mice per isolation method). Live cells/intact nuclei were identified and purified using flow cytometry. For the cells, either live $\mathrm{CD} 45^{+}$, live $\mathrm{CD} 45^{-}$or live hepatocytes were sorted. 1 ex vivo digested sample and 1 in vivo digested sample were also stained with a panel of 107 (ex vivo cells) or 161 (in vivo cells) oligo-conjugated antibodies for CITE-seq analysis. FACS-purified cells/nuclei were loaded onto the 10x chromium platform and scRNA-seq, CITE-seq, or snRNA-seq performed. Following clean up and QC, cells from the same mice were pooled together in the same ratios $\left(\mathrm{CD} 45^{+}: \mathrm{CD} 45^{-}: \mathrm{Heps}\right)$ as found in the tissue as a whole before sorting, different mice were then pooled together and the data were analyzed using scVl.

(A-C) UMAPs showing annotations of cell types and proportions of each cell type as a $\%$ of total cells in the UMAP isolated using (A) ex vivo digestion; 13,144 cells, (B) in vivo digestion; 24,014 cells and (C) nuclei; 8,583 nuclei.

(D) Average number of genes/cell in the annotated mac, B cell, hepatocyte, endothelial, and stromal cell populations following each isolation method. ${ }^{*} p<0.05$ one-way ANOVA with Bonferroni post-test per cell type.

(E) Correlation plot showing genes captured within the mac population when the liver is digested with the in vitro versus the in vivo digestion protocol.

(F) Correlation plots showing genes captured within the mac, endothelial cell, and hepatocyte populations when cells are isolated using the in vivo digestion protocol or nuclei are isolated.

(G-L) Confocal microscopy images to determine true abundance of (G) stromal cells and cholangiocytes (H) endothelial cells, (I) macs, (J) dendritic cells, (K) B cells, and (L) T cells in vivo. Scale bars, $200 \mu \mathrm{m}$.

(M) The percentage of each population was calculated based on the percentage of a given population divided by the total number of nuclei. A threshold was applied to the DAPI channel (picture 1) in ImageJ (picture 2) and nuclei were automatically counted based on the ImageJ "analyze particles" plugin (size ). Due to the density of some liver zones, some nuclei were not automatically counted (arrow, picture 3). Those were then manually counted and added to the total number of nuclei. For the populations of interest, cells were counted manually based on specific markers (for example, CD3 for T cells, picture 4). Counting was performed blinded prior to analysis of the sequencing results.

(N) Proportion of indicated cell types as a \% of total cells identified in confocal microscopy images. Data are from 3-7 images per cell type taken from 2-4 mice. 

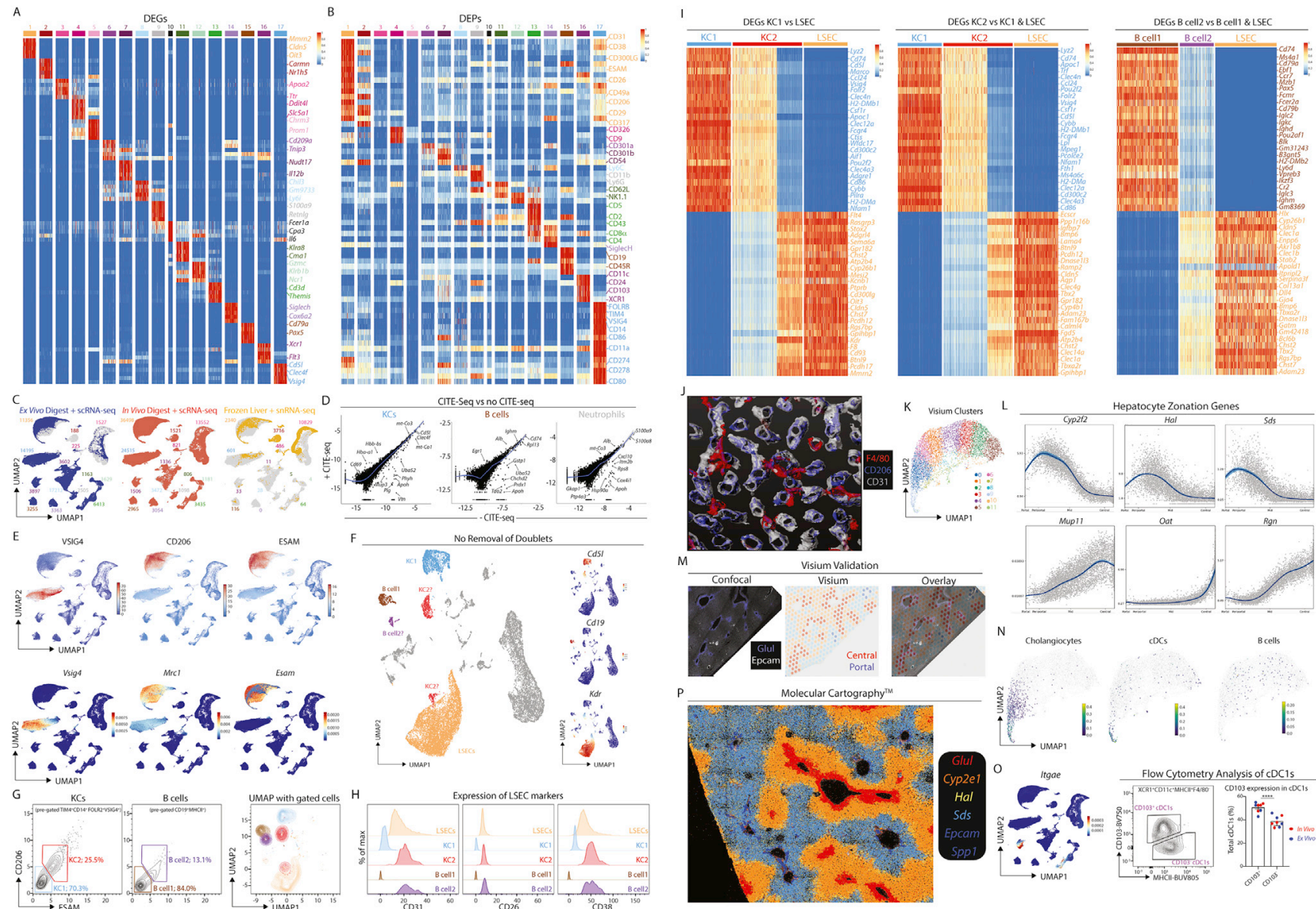

Figure S2. Combination of CITE-seq, scRNA-seq, snRNA-seq, and spatial analyses enables identification of all hepatic cell types including bona fide cell doublets, related to Figure 1

(A and B) Top DEGs (A) and DEPs (B) for cell types from Figure 1B.

(C) Distinct profiles of cells or nuclei within the UMAP depending on isolation protocols; 71,162 cells from ex vivo digestions, 96,066 cells from in vivo digestions, and 18,666 nuclei. Numbers on plots represent numbers of cells/nuclei per population.

(D) Correlation plots showing genes captured within the KC, B cell and neutrophil populations with and without addition of CITE-seq antibodies.

(E) Expression of VSIG4, CD206, and ESAM (protein, top) and Vsig4, Mrc1, and Esam (mRNA, bottom).

(F) UMAP showing clusters of cells when only minimal QC for gene number and \% mitochondrial genes is performed; 17,669 cells pooled from 3 samples. Expression of $C d 51, C d 19$, and $K d r$ by the clusters facilitating identification of cell types per annotation.

(G) CITE-seq data from (F) in Flow-Jo showing expression of CD206 and ESAM in total KCs (left) and total B cells (middle). Numbers represent \% of entire KC or B cell population. Identified populations were then mapped back onto the original UMAP (right).

(H) Expression of CD31, CD26, and CD38 by indicated populations.

(I) Heatmaps showing expression of top DEGs between KC1s and LSECs (left), KC2s and KC1s + LSECs (middle) and B cell2s and B cell1s + LSECs (right).

(J) 3D reconstruction of murine liver following perfusion with antigen fix to inflate endothelial cells and staining with antibodies against CD31, CD206, and F4/80.

(K) UMAP showing clusters generated from Visium analysis of liver tissue (4 samples) and liver capsule (1 sample).

(L) Top unbiased genes defining zonation trajectory from portal to central vein in Visium.

(M) Expression of Glul and Epcam by confocal microscopy (left), annotation of portal, periportal, mid, and central regions on same tissue section (middle) and overlay of both datasets (right).

(N) Identification of cholangiocyte (left) and cDC (right) signatures on zonated Visium spots.

(P) Molecular Cartography showing expression of indicated zonated hepatocyte mRNAs in liver tissue. Data are representative of 2 mice.

(O) Expression of Itgae (encoding CD103) in the UMAP of the total liver (left) and flow cytometric analysis of total cDC1s for CD103 and MHCII expression in the healthy murine liver (right). 
A

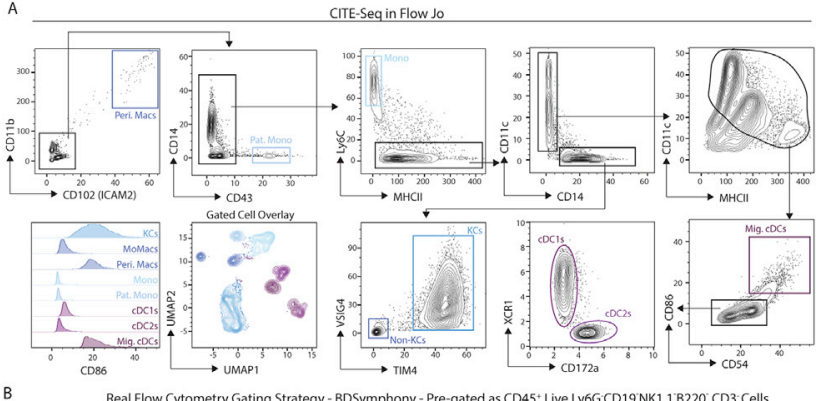

B Real Flow Cytometry Gating Strategy - BDSymphony - Pre-gated as CD45 L Live Ly6G CD 19 NK1.1 B B220 CD3 Cells
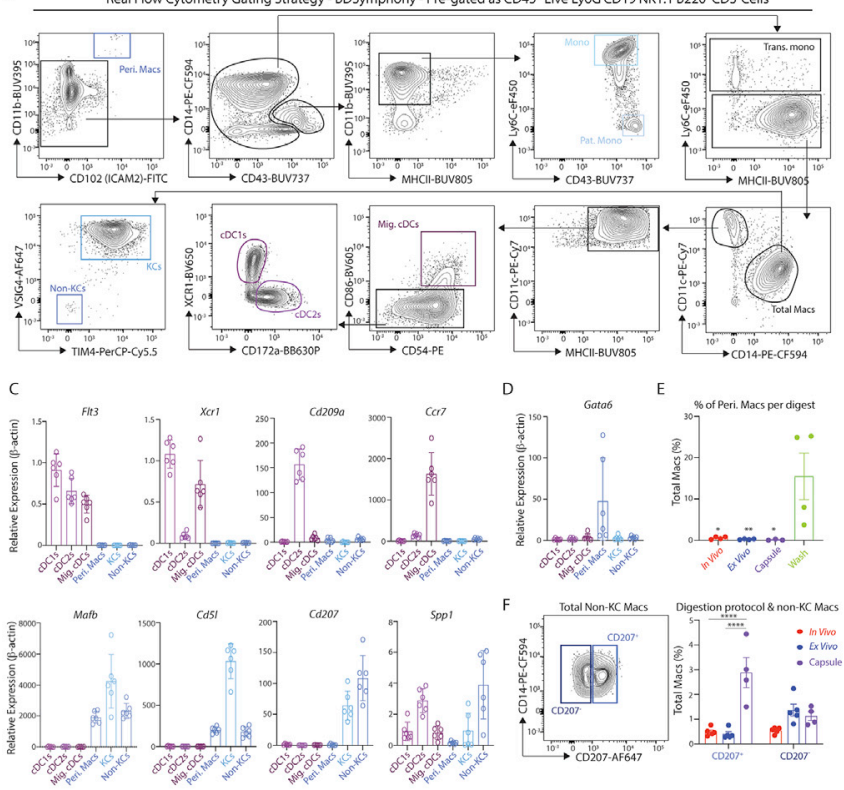

G
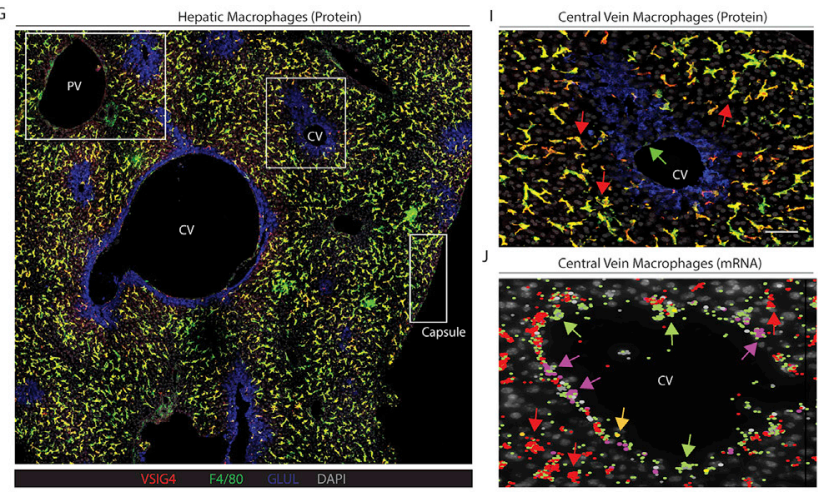

Central Vein Macrophages (mRNA)
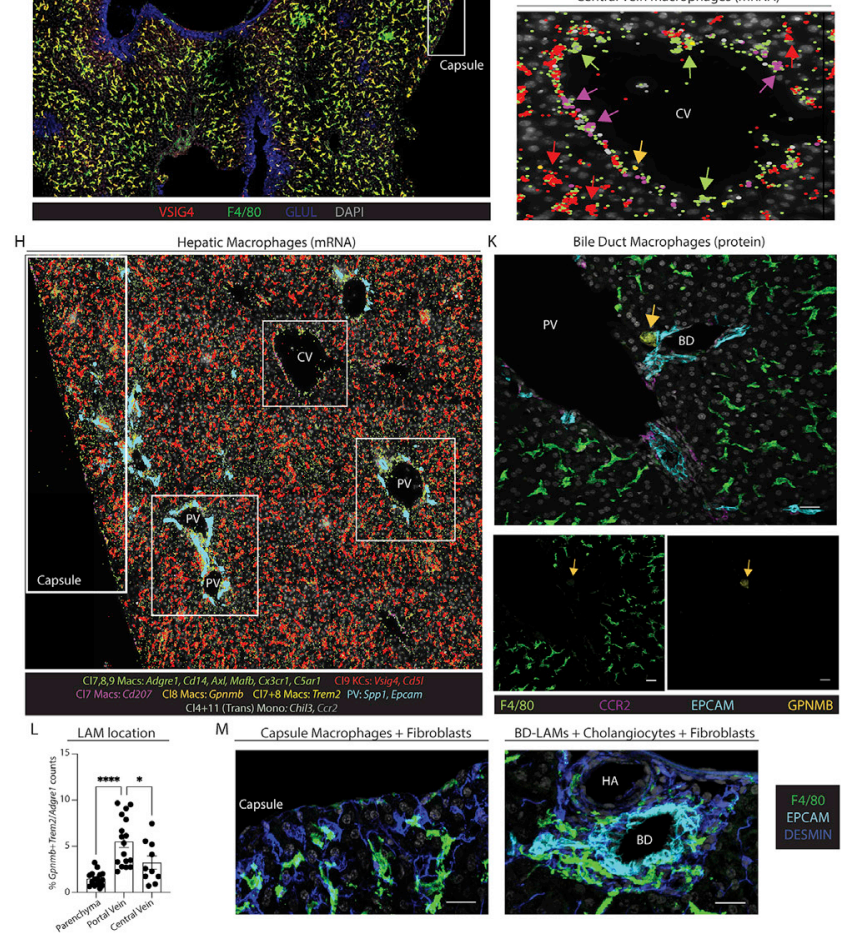

Figure S3. Validated flow cytometry gating strategy for murine myeloid cells, related to Figure 2

(A) CITE-seq data from the murine myeloid cells in Figure $2 \mathrm{~A}$ were exported as an FCS file and an in silico gating strategy identified in FlowJo software.

(B) Application of the in silico gating strategy with a 21-color flow cytometry panel. Myeloid cells were pre-gated as live CD45 lineage cells (Ly6G $\left.{ }^{-} \mathrm{CD}_{19}^{-} \mathrm{NK} 1.1^{-} \mathrm{B} 220^{-} \mathrm{CD}^{-}\right)$. Data are representative of 3 experiments with 3-6 mice per experiment.

(C) cDC1s, cDC2s, migratory cDCs (Mig. cDCs), peritoneal macs (Peri. Macs), KCs, and non-KC macs (non-KCs) were FACS-purified using gating strategy in (B), mRNA was isolated and qPCR performed to examine expression of indicated genes defining each population to validate their identity. Data are representative of 2 experiments with $n=3-6$.

(D) Putative peritoneal macs were FACS-purified using gating strategy in (B) and expression of Gata6 was examined by qPCR compared with other hepatic myeloid populations. Data are from a single experiment with $\mathrm{n}=6$.

(E) Peritoneal macs as a \% of total macs recovered from the liver using different digestion techniques (in vivo, ex vivo, or capsule) or in supernatants in which livers were washed following removal from the mouse but prior to digestion (wash). Data are from a single experiment with $n=4$. ${ }^{*} p<0.05$, ${ }^{\star \star} p<0.01$ one-way ANOVA with Bonferroni post-test compared with wash data.

(F) Expression of CD14 and CD207 within the non-KC mac population from (B) (left) and \% of CD207 ${ }^{+}$and CD207 $^{-}$populations among total macs in livers digested using the ex vivo or in vivo protocols or in dissected and digested liver capsule (right). Data are representative of two experiments with $\mathrm{n}=4-5 \mathrm{mice}$ per experiment. ${ }^{\star \star \star \star} \mathrm{p}<0.0001$ mixed effects analysis with Tukey's multiple comparison test.

(G) Expression of VSIG4, F4/80, GLUL, and DAPI by confocal microscopy. Insets represent zones featured in Figures 2E, 2G, and S3I.

(H) Molecular Cartography of indicated genes and cell types. Insets represent zones featured in Figures $2 \mathrm{~F}, 2 \mathrm{H}$, and $\mathrm{S} 3 \mathrm{~J}$.

(I) Expression of VSIG4, F4/80, GLUL, and DAPI by confocal microscopy at the central vein. Scale bars, $50 \mu \mathrm{m}$.

(J) Molecular Cartography of indicated genes and cell types at central vein.

(K) Expression of F4/80, EPCAM, CCR2, GPNMB, and DAPI by confocal microscopy at a portal vein (top) or F4/80 or GPNMB alone (bottom). Scale bars, $25 \mu \mathrm{m}$. (L) Quantification of \% of Gpnmb \& Trem2 counts over Adgre1 counts in indicated regions of tissue as assessed using Molecular Cartography data. Each dot represents an individual region. ${ }^{*} p<0.05,{ }^{* \star *} p>0.0001$ one-way ANOVA with Bonferroni post-test.

(M) Expression of DESMIN and F4/80 at the liver capsule and underlying parenchyma (left) or EPCAM, DESMIN and F4/80 at the bile duct by confocal microscopy. PV, portal vein; CV, central vein; HA, hepatic artery; BD, bile duct. Arrows indicate specific cell types, where color corresponds to cell type/markers. All images are representative of 2-6 mice. 

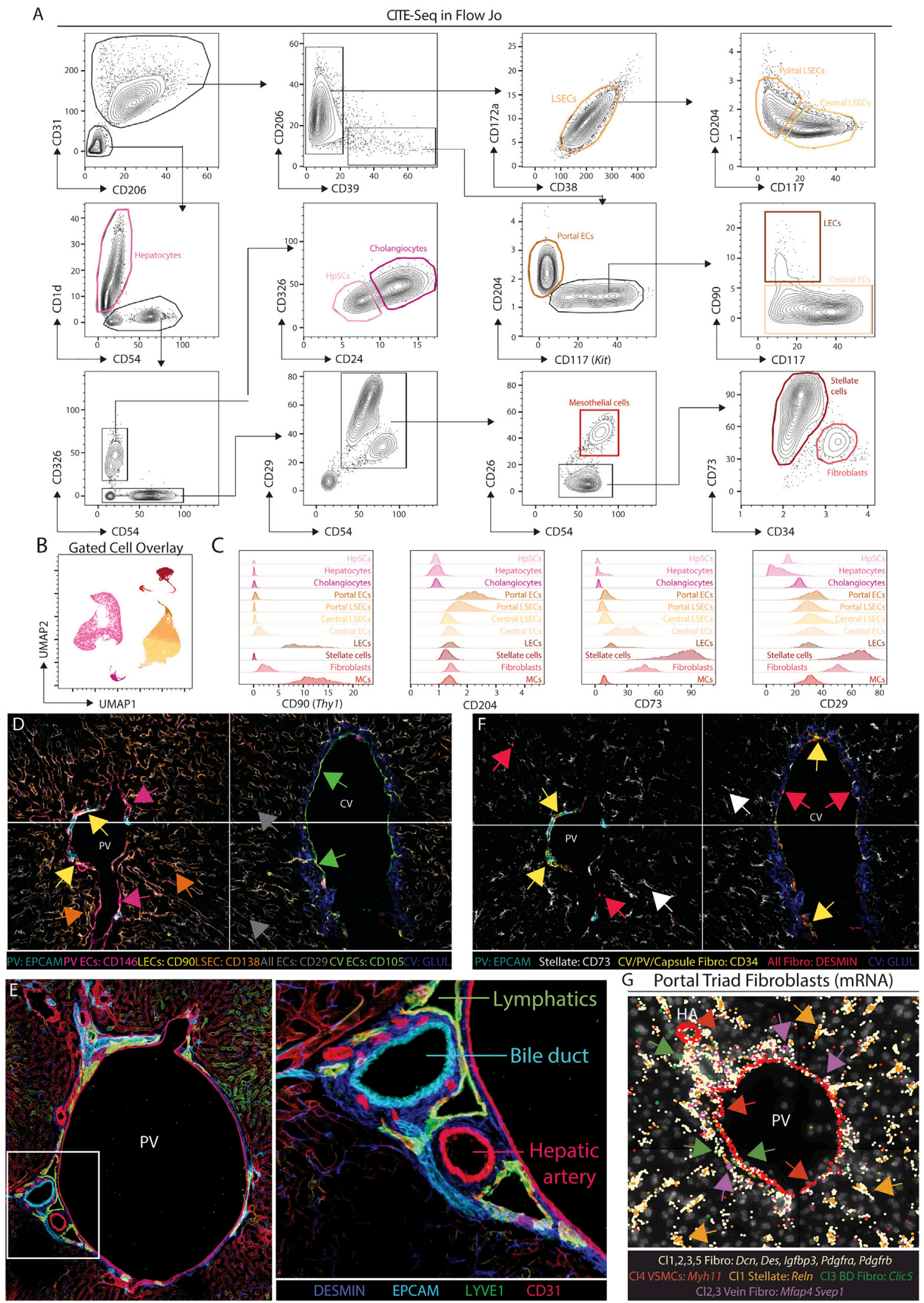
Figure S4. Protein markers of murine $\mathrm{CD} 45^{-}$cell subsets, related to Figure 3

(A) CITE-seq data from the murine CD45- cells in Figure 3 A were exported as an FCS file and an in silico gating strategy identified in FlowJo.

(B) Gated cell overlay of populations identified using strategy in (A).

(C) Expression of CD90, CD204, CD73, and CD29 markers by indicated cell types.

(D) Expression of indicated protein markers in 60-plex MICS analysis in endothelial cells.

(E) Expression of DESMIN, EPCAM, LYVE1, and CD31 at a portal triad (left) with inset (right).

(F) Expression of indicated protein markers in 60-plex MICS analysis in stromal cells.

(G) Molecular Cartography of indicated genes and cell types at portal vein. PV, portal vein; CV, central vein. Arrows indicate specific cell types, where color corresponds to cell type/markers. All images are representative of 2-6 mice. 

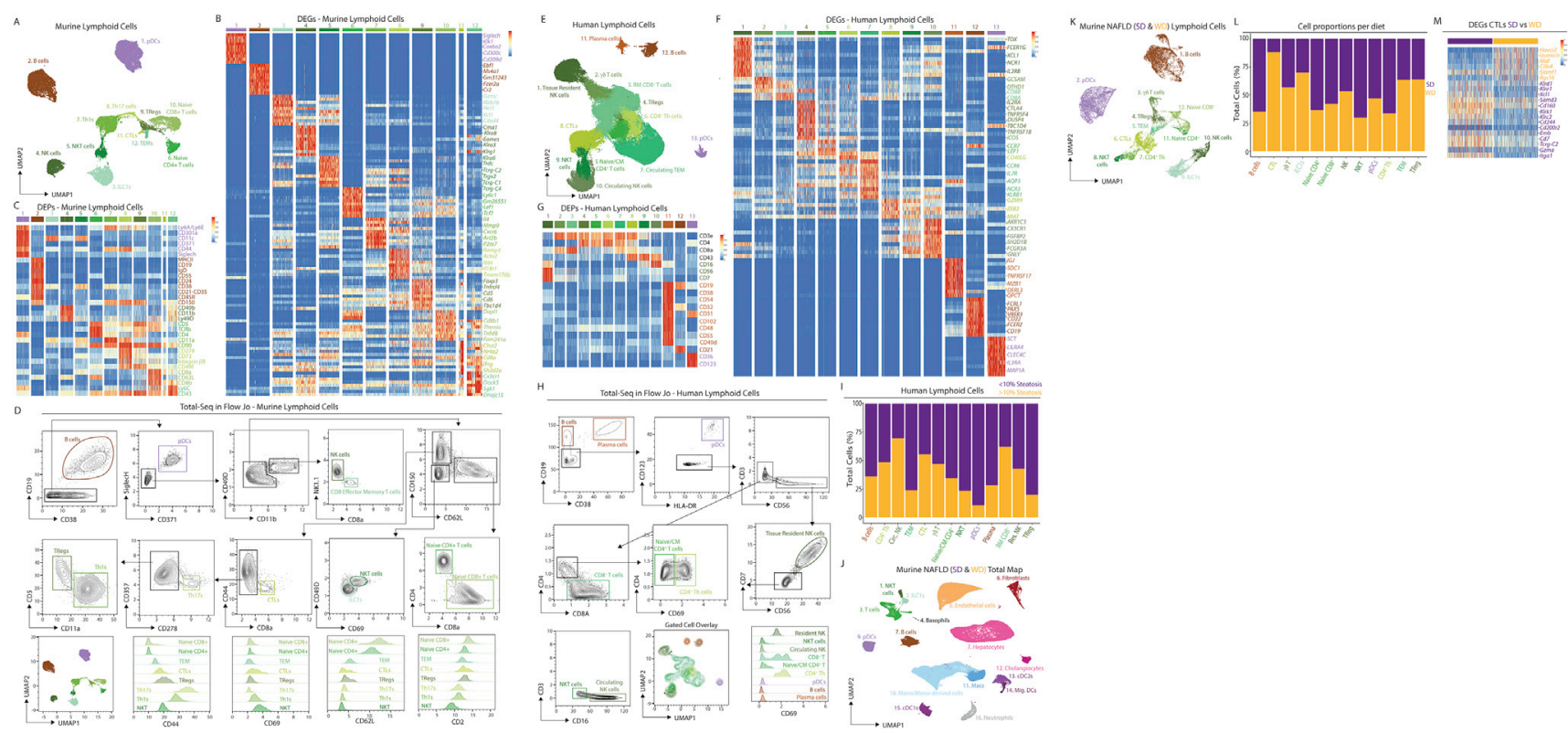

Figure S5. Combination of CITE-seq, scRNA-seq, snRNA-seq, and spatial analyses enables generation of a human liver atlas, related to Figure 4

(A) Murine lymphoid cells (B cells, T cells, NK cells, ILC1s, pDCs; 27,398 cells) were isolated from Figure 1B and re-clustered with TotalVI.

(B and C) Top DEGs (B) and DEPs (C) for the cell types from Figure S5A.

(D) CITE-seq data from Figure S5A were exported as an FCS file and an in silico gating strategy identified in FlowJo.

(E) Human lymphoid cells (B cells, T cells, NK cells, ILC1s, pDCs; 105,790 cells) were isolated from Figure 4B and re-clustered with TotalVI.

( $F$ and $G)$ Top DEGs $(F)$ and DEPs $(G)$ for the cell types from Figure S5E.

(H) CITE-seq data from Figure S5E were exported as an FCS file and an in silico gating strategy identified in FlowJo.

(I) Proportion of indicated cell types arising from patients with $<10 \%$ (purple) or $>10 \%$ steatosis (yellow).

(J) Hepatic cells were isolated from 22 C57B/l6 mice fed either a standard diet (SD) or a western diet (WD) for 24 or 36 weeks to induce NAFLD and NASH by ex vivo (10 samples) or in vivo (12 samples) enzymatic digestion. Alternatively, livers were snap frozen and nuclei isolated by tissue homogenization (14 samples). Live cells/intact nuclei were purified using FACS. For cells, total live, live CD45 , live CD45-, live hepatocytes or myeloid cells (live CD45 ${ }^{+}, \mathrm{CD}^{-}, \mathrm{CD}^{-} 9^{-}, \mathrm{B220}^{-}$, NK1.1 ${ }^{-}$) were sorted. 10 samples were also stained with a panel of 107-161 barcode-labeled antibodies for CITE-seq analysis. All datasets were pooled together and after QC 121,980 cells/nuclei were clustered using TotalVI.

(K) Murine lymphoid cells (B cells, T cells, NK cells, ILC1s, pDCs; 21,322 cells) from mice fed the SD or WD for 24 or 36 weeks were isolated from Figure S5J and re-clustered with TotalVI.

(L) Proportion of indicated cell types arising from mice fed the SD (purple) or WD (yellow).

(M) Top DEGs between CTLs isolated from mice fed the SD (purple) or WD (yellow). 

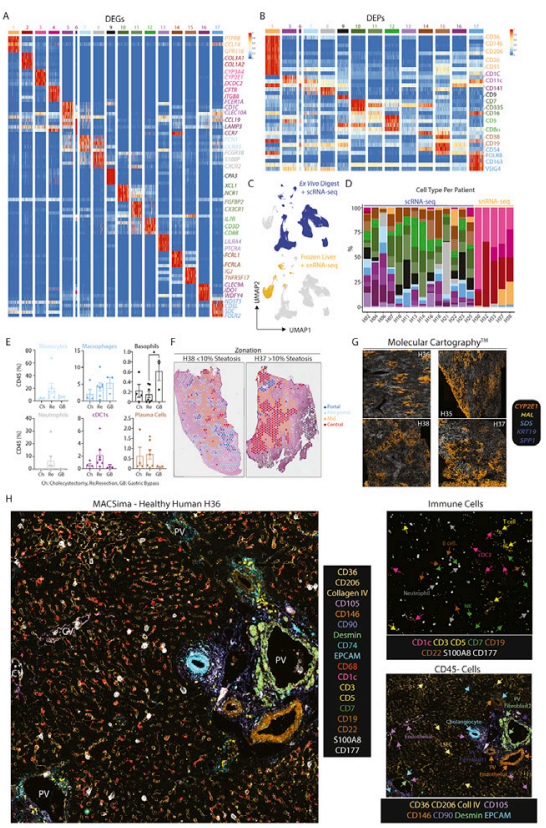
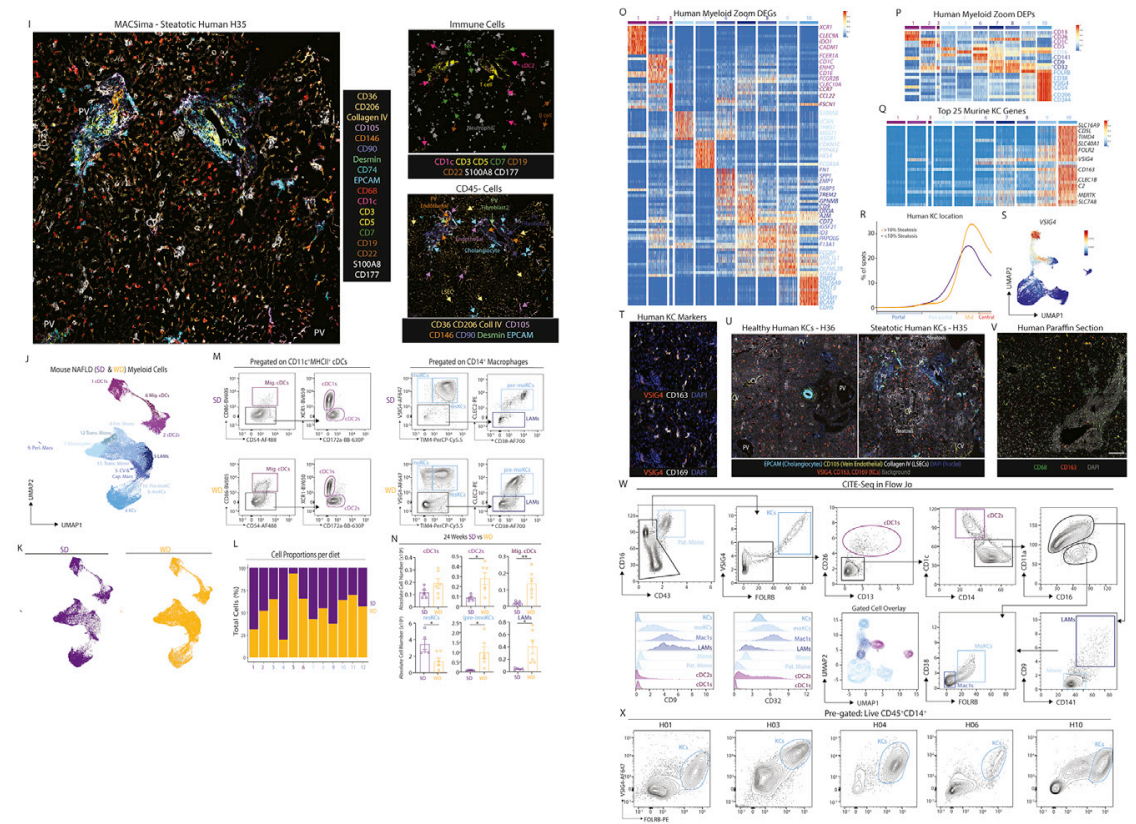

Figure S6. Combination of CITE-seq, scRNA-seq, snRNA-seq, and spatial analyses enables generation of a human liver atlas and identification of bona fide human $\mathrm{KCs}$, related to Figure 4

(A and B) Top DEGs (A) and DEPs (B) for the cell types from Figure 4B.

(C) Distinct profiles of cells or nuclei within the UMAP depending on isolation protocol used; 152,535 cells from ex vivo digestions and 15,063 nuclei.

(D) Proportion of each cell type per patient profiled.

(E) Proportion of indicated cell types as a \% of total CD45 cells calculated from ex vivo digested samples per surgery type. Ch; cholecystectomy, Re; resection,

GB; gastric bypass. ${ }^{*} \mathrm{p}<0.05$; one-way ANOVA with Bonferroni post-test.

(F) Mapping of Visium UMAP zonation patterns onto tissue sections from patient $\mathrm{H} 35$ and $\mathrm{H} 37$.

(G) Expression of indicated zonation genes in patients $\mathrm{H} 35-\mathrm{H} 38$ assessed by Molecular Cartography.

( $\mathrm{H}$ and I) Expression of indicated proteins by MICS 100-plex protein analysis in the healthy $(\mathrm{H})$ and steatotic (I) human liver.

(J) Murine myeloid cells (cDC1s, cDC2s, Mig. cDCs, Macs, monocytes, and monocyte-derived cells; 42,922 cells) from mice fed the SD or WD for 24 or 36 weeks were isolated from Figure $\mathrm{S} 5 \mathrm{~J}$ and re-clustered with TotalVI.

(K) Distribution of cells in UMAP originating from SD- (purple) or WD- (yellow) fed mice.

(L) Proportion of indicated cell types arising from mice fed the SD (purple) or WD (yellow).

( $M$ and N) Flow cytometry analysis of indicated cell populations in SD and WD-fed mice (24 weeks). Representative gating strategies (M) and absolute number of indicated populations $(N)$. ${ }^{*} p<0.05,{ }^{* *} p<0.01$ Student's $t$ test. Data are from 2 independent experiments with $n=5-6$ per diet.

$(\mathrm{O}$ and $\mathrm{P})$ Top DEGs $(\mathrm{O})$ and DEPs $(\mathrm{P})$ for cell types from Figure $4 \mathrm{H}$.

(Q) Top 25 Murine KC genes as expressed by the human myeloid cell clusters.

(R) Mapping of KC signature onto Visium trajectory for healthy (purple) and steatotic (orange) livers.

(S) Expression of VSIG4 mRNA within human myeloid cells.

(T) Expression of VSIG4 (red) and CD163 (gray, top) or CD169 (gray, bottom) by MICS analysis in healthy human liver.

(U) Representative images showing KC location (red) as assessed by MICS analysis in the healthy (left) and steatotic (right) human liver. PV, portal vein; CV, central Vein, dashed line indicates zones of steatosis.

(V) Representative image of CD68 and CD163 staining in 10-15-year-old human liver paraffin sections. Image is representative of 6 different patients.

(W) In silico gating strategy to isolate distinct myeloid cell populations identified from CITE-seq data.

(X) Expression of VSIG4 and FOLR2 by live CD45 ${ }^{+}$cells also expressing CD14 in indicated human liver biopsies by flow cytometry. Data are representative of 21 biopsy samples analyzed. 


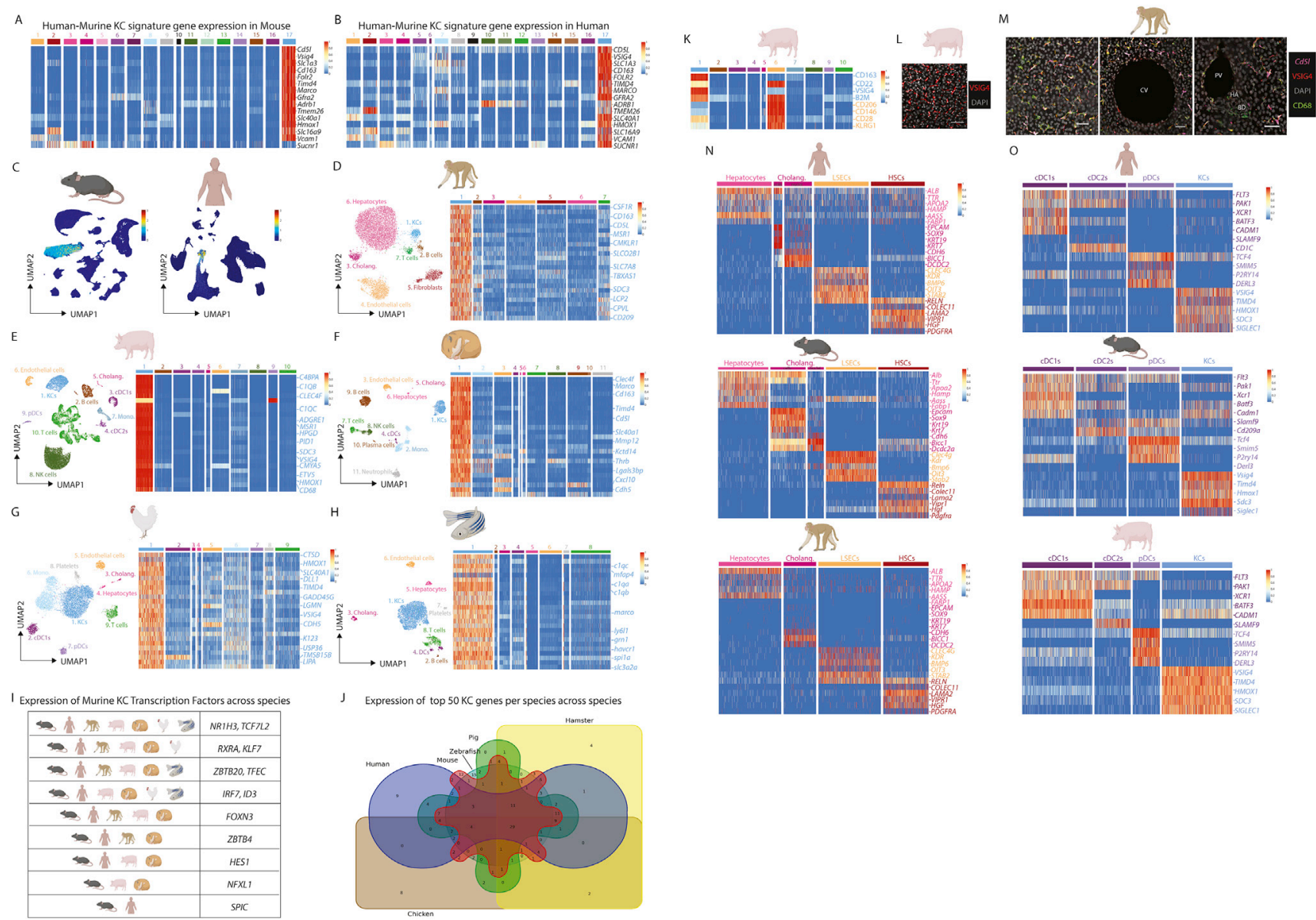

Figure S7. Conserved and unique features of KCs across species, related to Figure 4

(A and B) Expression of human-murine $\mathrm{KC}$ signature genes across cell types in mouse (A) and human (B).

(C) Unbiased identification of KCs in mouse and human using the human-murine KC signature and the signature finder algorithm (Pont et al., 2019).

(D-H) Annotated UMAPs from indicated species and expression of top KC-specific genes compared with other cells per species.

(I) Expression of previously identified core murine transcription factors (Bonnardel et al., 2019) by KCs across species.

(J) Venn diagram showing convergence and divergence of expression of top $50 \mathrm{KC}$ genes per species across species, see Table S9 for genes lists per species.

(K) Top DEPs (identified with cross reactive human antibodies) in the pig CITE-seq data.

(L) Expression of VSIG4 in the porcine liver by confocal microscopy.

(M) Expression of VSIG4, CD68 (protein), and CD5L (mRNA) in macaque liver. PV, portal vein; HA, hepatic artery; BD, bile duct. All images are representative of 2 livers.

$(\mathrm{N}$ and $\mathrm{O})$ Conserved expression of indicated genes across $\mathrm{CD}_{4} 5^{-}(\mathrm{N})$ and $\mathrm{CD}_{4} 5^{+}(\mathrm{O})$ cell types and species. 


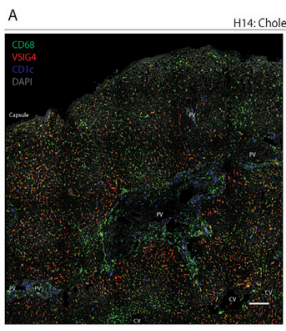

B

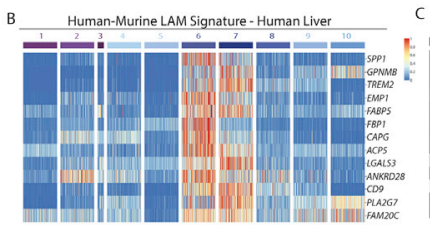

D
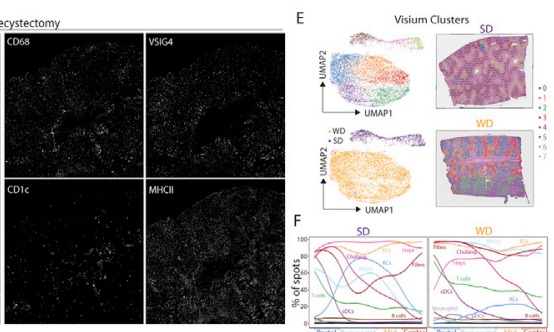

C Human-Murine LAM Signature - Mouse Liver
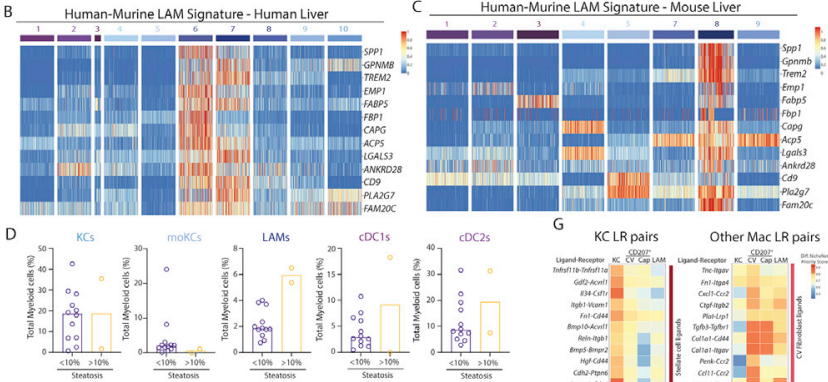

HACRELT 11 GDFPBSMPTO

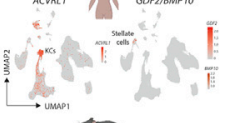

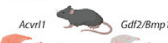

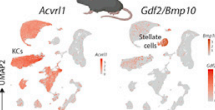

$\rightarrow$

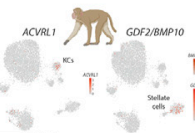
Acull $7^{\text {na }}$

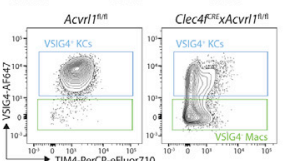

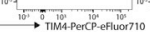
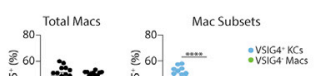

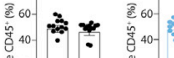

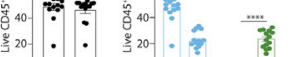

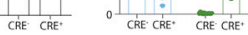
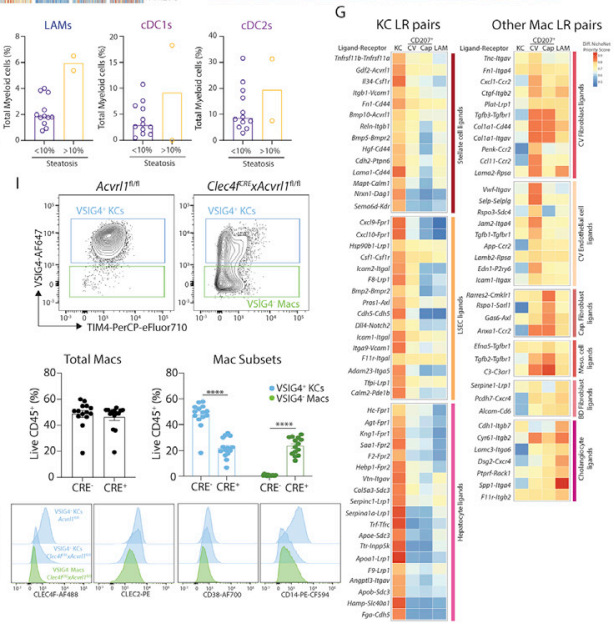

J

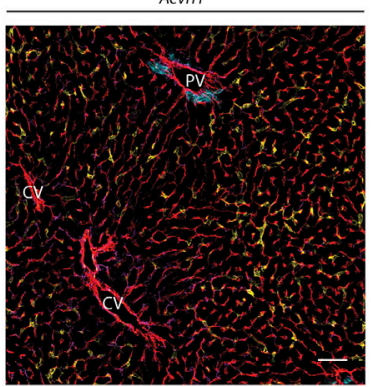

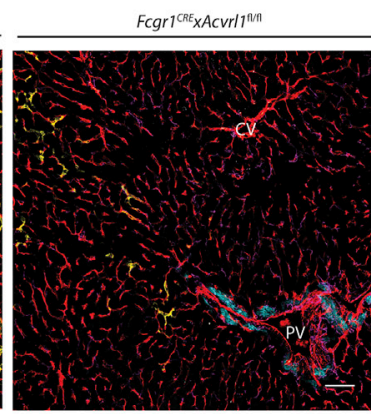

Figure S8. Evolutionarily conserved signals regulate LAM and KC development, related to Figures 5 and 7

(A) Confocal microscopy of healthy human liver showing expression of indicated markers. Scale bars, $200 \mu \mathrm{m}$.

( $B$ and $C$ ) Expression of conserved human-murine bile-duct LAM signature in human (B) and mouse (C) hepatic myeloid cells.

(D) Proportion of indicated myeloid cell populations as a \% of total myeloid cells in human liver biopsies profiled by scRNA-seq when divided based on presence of steatosis.

(E) Mice were fed a western diet (WD) or standard diet (SD) for 36 weeks to induce NAFLD and Visium analysis was performed. Analysis is pooled from 1 liver slice from the SD condition and 3 liver slices from the WD condition. Shown are cluster and sample annotations.

(F) Zonation of all cell types from Figure S5J in murine NAFLD map (SD\&WD).

(G) Differential NicheNet highlighting prioritized conserved (human-mouse) ligand-receptor (LR) pairs between indicated macs and their niche cells. LR pairs are grouped according to the niche cell type with highest ligand expression.

(H) Expression of ALK1 (ACVRL1), BMP9 (GDF2), and BMP10 in human, mouse, and macaque livers where both KCs and stellate cells were profiled.

(I) Livers were harvested from Clec4f-CrexAcvrl fl/ffl mice or Acvrl $1^{f / f l}$ controls and KCs examined (top) and quantified (middle) using VSIG4 expression. Expression of indicated KC markers by mac populations in Clec4f-CrexAcvrl1 ${ }^{\mathrm{fl} / \mathrm{fl}}$ or Acvrl $1^{\mathrm{fl} / \mathrm{fl}}$ control mice (bottom). Data are pooled from 2 independent experiments with $n=14$ per group. Student's t test. ${ }^{* \star \star *} p<0.0001$.

(J) Expression of CD31 (ECs), DESMIN (stromal cells), F4/80 (Macs), and EPCAM (cholangiocytes) by confocal microscopy in Fcgr1-CrexAcvrl1 ${ }^{\text {fl/fl }}$ mice and Acvrl1 ${ }^{+/+}$controls. PV, portal vein; CV, central vein. Images are representative of 2 mice per group. 